\title{
On the Rim Tori Refinement of Relative Gromov-Witten Invariants
}

\author{
Mohammad F. Tehrani and Aleksey Zinger*
}

July 12, 2018

\begin{abstract}
We construct Ionel-Parker's proposed refinement of the standard relative Gromov-Witten invariants in terms of abelian covers of the symplectic divisor and discuss in what sense it gives rise to invariants. We use it to obtain some vanishing results for the standard relative GromovWitten invariants. In a separate paper, we describe to what extent this refinement sharpens the usual symplectic sum formula and give further qualitative applications.
\end{abstract}

\section{Contents}

1 Introduction 2

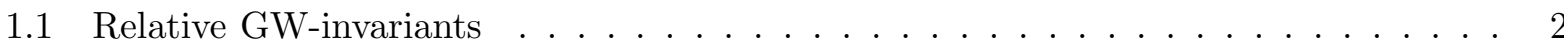

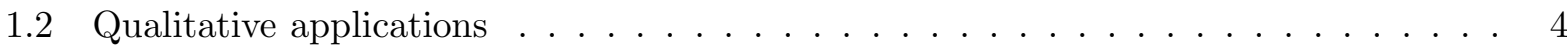

1.3 Outline of the paper $\ldots \ldots \ldots \ldots \ldots \ldots \ldots$

2 General topological context 7

2.1 Complement of submanifold . . . . . . . . . . . . . . . . . 7

2.2 Splice of two manifolds . . . . . . . . . . . . . . . . 8

3 Cutting out a submanifold $\quad \mathbf{1 0}$

3.1 Changes in homology . . . . . . . . . . . . . . . . . 10

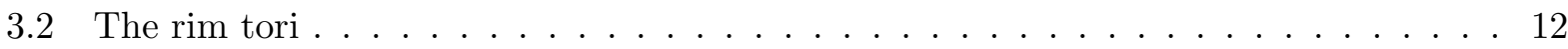

4 Gluing along a common submanifold $\quad 14$

4.1 Changes in homology . . . . . . . . . . . . . . . . . . 15

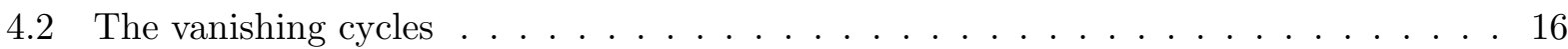

4.3 Changes in $\operatorname{rim}$ tori . . . . . . . . . . . . . . . . . . . . 20

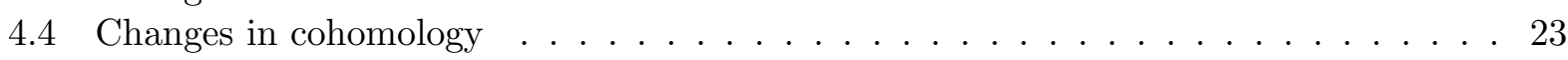

5 Abelian covers of topological spaces $\quad 26$

5.1 Notation and examples . . . . . . . . . . . . . . . . . . . 26

5.2 Some properties. . . . . . . . . . . . . . . . . 30

*Partially supported by NSF grant 0846978 
6 The refined relative GW-counts 34

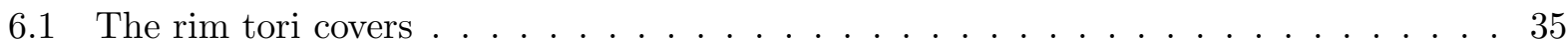

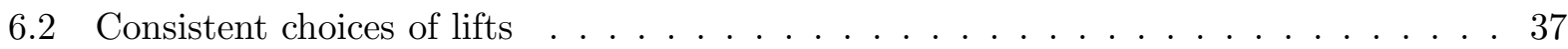

6.3 Proof of Theorem $1.1 \ldots \ldots \ldots \ldots \ldots \ldots \ldots$

\section{Introduction}

Gromov-Witten invariants of symplectic manifolds, which include nonsingular projective varieties, are certain counts of pseudo-holomorphic curves that play prominent roles in symplectic topology, algebraic geometry, and string theory. The decomposition formulas, known as symplectic sum formulas in symplectic topology and degeneration formulas in algebraic geometry, are one of the main tools used to compute Gromov-Witten invariants; they relate Gromov-Witten invariants of one symplectic manifold to Gromov-Witten invariants of two simpler symplectic manifolds. Unfortunately, the formulas of [10, 11] do not completely determine the former in terms of the latter in many cases because of the so-called vanishing cycles: second homology classes in the first manifold which vanish when projected to the union of the other two manifolds; see (2.2). A refinement to the usual relative Gromov-Witten invariants of [9, 11] is sketched in [7]; the aim of this refinement is to resolve the unfortunate deficiency of the formulas of [10, 11] in [8]. In this paper, we formally construct the refinement of [7, discuss the invariance and computability aspects of the resulting curve counts, and obtain some vanishing results for the usual relative GWinvariants. In the sequel [4], we describe the applicability of this refinement to computing the Gromov-Witten invariants of symplectic sums and obtain further qualitative applications.

\section{$1.1 \quad$ Relative GW-invariants}

Let $(X, \omega)$ be a compact symplectic manifold and $J$ be an $\omega$-tame almost complex structure on $X$. For $g, k \in \mathbb{Z}^{\geq 0}$ and $A \in H_{2}(X ; \mathbb{Z})$, we denote by $\overline{\mathfrak{M}}_{g, k}(X, A)$ the moduli space of stable $J$-holomorphic $k$-marked degree $A$ maps from connected nodal curves of genus $g$. By [12, 5, 2, this moduli space carries a virtual class, which is independent of $J$ and of representative $\omega$ in a deformation equivalence class of symplectic forms on $X$. If $V \subset X$ is a compact symplectic divisor (symplectic submanifold of real codimension 2$), \ell \in \mathbb{Z}^{\geq 0}, \mathbf{s} \equiv\left(s_{1}, \ldots, s_{\ell}\right)$ is an $\ell$-tuple of positive integers such that

$$
s_{1}+\ldots+s_{\ell}=A \cdot V,
$$

and $J$ restricts to an almost complex structure on $V$, let $\overline{\mathfrak{M}}_{g, k ; \mathbf{s}}^{V}(X, A)$ denote the moduli space of stable $J$-holomorphic $(k+\ell)$-marked maps from connected nodal curves of genus $g$ that have contact with $V$ at the last $\ell$ marked points of orders $s_{1}, \ldots, s_{\ell}$. According to [11, 9], this moduli space carries a virtual class, which is independent of $J$ and of representative $\omega$ in a deformation equivalence class of symplectic forms on $(X, V)$.

There are natural evaluation morphisms

$$
\begin{aligned}
\mathrm{ev}_{X} \equiv \mathrm{ev}_{1} \times \ldots \times \mathrm{ev}_{k}: \overline{\mathfrak{M}}_{g, k}(X, A), \overline{\mathfrak{M}}_{g, k ; \mathbf{s}}^{V}(X, A) \longrightarrow X^{k} \\
\mathrm{ev}_{X}^{V} \equiv \mathrm{ev}_{k+1} \times \ldots \times \mathrm{ev}_{k+\ell}: \overline{\mathfrak{M}}_{g, k ; \mathbf{s}}^{V}(X, A) \longrightarrow V_{\mathbf{s}} \equiv V^{\ell},
\end{aligned}
$$

sending each stable map to its values at the marked points. The (absolute) GW-invariants of $(X, \omega)$ are obtained by pulling back elements of $H^{*}\left(X^{k} ; \mathbb{Q}\right)$ by the morphism $(1.2)$ and integrating them 
and other natural classes on $\overline{\mathfrak{M}}_{g, k}(X, A)$ against the virtual class of $\overline{\mathfrak{M}}_{g, k}(X, A)$. The (relative) GW-invariants of $(X, V, \omega)$ are obtained by pulling back elements of $H^{*}\left(X^{k} ; \mathbb{Q}\right)$ and $H^{*}\left(V_{\mathbf{s}} ; \mathbb{Q}\right)$ by the morphisms (1.2) and (1.3), and integrating them and other natural classes on $\overline{\mathfrak{M}}_{g, k ; \mathbf{s}}^{V}(X, A)$ against the virtual class of $\overline{\mathfrak{M}}_{g, k ; \mathbf{s}}^{V}(X, A)$.

As emphasized in [7, Section 5], two preimages of the same point in $V_{\mathbf{s}}$ under (1.3) determine an element of

$$
\mathcal{R}_{X}^{V} \equiv \operatorname{ker}\left\{\iota_{X-V *}^{X}: H_{2}(X-V ; \mathbb{Z}) \longrightarrow H_{2}(X ; \mathbb{Z})\right\}
$$

where $\iota_{X-V}^{X}: X-V \longrightarrow X$ is the inclusion; see Section 2.1. The elements of $\mathcal{R}_{X}^{V}$, called rim tori in [7], can be represented by circle bundles over loops $\gamma$ in $V$; see Section 3.1. By standard topological considerations,

$$
\mathcal{R}_{X}^{V} \approx H_{1}(V ; \mathbb{Z})_{X} \equiv \frac{H_{1}(V ; \mathbb{Z})}{H_{X}^{V}}, \quad \text { where } \quad H_{X}^{V} \equiv\left\{A \cap V: A \in H_{3}(X ; \mathbb{Z})\right\} ;
$$

see Corollary 3.2 .

The main claim of [7, Section 5] is that the above observations can be used to lift (1.3) over some regular (Galois), possibly disconnected (unramified) covering

$$
\pi_{X ; \mathbf{s}}^{V}: \widehat{V}_{X ; \mathbf{s}} \longrightarrow V_{\mathbf{s}}
$$

though the topology of this cover is not specified and the group of its deck transformation is described incorrectly as $\mathcal{R}_{X}^{V}$ in [7]. Since

$$
\operatorname{ev}_{X}^{V}=\pi_{X ; \mathbf{s}}^{V} \circ \widetilde{e v}_{X}^{V}: \overline{\mathfrak{M}}_{g, k ; \mathbf{s}}^{V}(X, A) \longrightarrow V_{\mathbf{s}}
$$

for some morphism

$$
\widetilde{\mathrm{ev}}_{X}^{V}: \overline{\mathfrak{M}}_{g, k ; \mathbf{s}}^{V}(X, A) \longrightarrow \widehat{V}_{X ; \mathbf{s}}
$$

the numbers obtained by pulling back elements of $H^{*}\left(\widehat{V}_{X ; \mathbf{s}} ; \mathbb{Q}\right)$ by $(1.8)$, instead of elements of $H^{*}\left(V_{\mathbf{s}} ; \mathbb{Q}\right)$ by $(1.3)$, and integrating them and other natural classes on $\overline{\mathfrak{M}}_{g, k ; \mathbf{s}}^{V}(X, A)$ against the virtual class of $\overline{\mathfrak{M}}_{g, k ; \mathbf{s}}^{V}(X, A)$ refine the usual GW-invariants of $(X, V, \omega)$. We will call these numbers the IP-counts for $(X, V, \omega)$.

The covering (1.6), which is completely determined by $(X, V)$ and $\mathbf{s}$, is defined in Section 6.1 based on the sketch in [7, Section 5] and after some preparation in Section [5.1. For example,

$$
\widehat{V}_{X ;()}=\mathcal{R}_{X}^{V} \times V_{()}=\mathcal{R}_{X}^{V},
$$

where ()$\in \mathbb{Z}_{+}^{0}$ is the empty vector. If $V$ is connected, then

$$
\widehat{V}_{X ; 1)}=\widehat{V}_{X}
$$

is the abelian covering corresponding to the quotient $H_{1}(V ; \mathbb{Z})_{X}$ of $H_{1}(V ; \mathbb{Z})$, i.e. to the preimage of $H_{X}^{V}$ under the Hurewicz homomorphism $\pi_{1}(X) \longrightarrow H_{1}(X ; \mathbb{Z})$. The group of deck transformations of the covering (1.6) is given by

$$
\operatorname{Deck}\left(\pi_{X ; \mathbf{s}}^{V}\right)=\frac{\mathcal{R}_{X}^{V}}{\mathcal{R}_{X ; \mathbf{s}}^{\prime V}} \times \mathcal{R}_{X ; \mathbf{s}}^{\prime V}
$$


for a certain submodule $\mathcal{R}_{X ; \mathbf{s}}^{\prime V}$ of $\mathcal{R}_{X}^{V}$. For example,

$$
\mathcal{R}_{X ; \mathbf{s}}^{\prime V}= \begin{cases}\{0\}, & \text { if } \ell=0 ; \\ \operatorname{gcd}(\mathbf{s}) \mathcal{R}_{X}^{V}, & \text { if }\left|\pi_{0}(V)\right|=1 .\end{cases}
$$

In general, the deck group (1.9) is different from $\mathcal{R}_{X}^{V}$ (contrary to an explicit statement in [7, Section 5]). By [14, Assertion 6] in the case $H_{1}(V ; \mathbb{Z})_{X}$ is of rank 1 and its extension [15], $H_{*}\left(\widehat{V}_{X ; \mathbf{s}} ; \mathbb{Q}\right)$ is not finitely generated if $V$ is connected, $\chi(V) \neq 0$, and $H_{1}(V ; \mathbb{Z})_{X}$ is not a torsion group (so that the covering (1.6) is infinite).

By standard covering spaces considerations, the total relative evaluation map (1.3) lifts over the covering (1.6); see Lemma 6.3. The lift (1.8) of (1.3) is not unique and involves choices of base points in various spaces. However, these choices can be made in a systematic manner and the lift (1.8) extends over the space of stable smooth maps (and $L_{1}^{p}$-maps with $p>2$ ); see Theorem 6.5 and Remark 6.7. This ensures that the IP-counts for $(X, V, \omega)$ are independent of $J$ and of representative $\omega$ in a deformation equivalence class of symplectic forms on $(X, V)$.

If $V=V_{1} \sqcup V_{2}$ for some symplectic divisors $V_{1}, V_{2} \subset X$, it is natural to consider the moduli spaces

$$
\overline{\mathfrak{M}}_{g, k ; \mathbf{s}_{1} \mathbf{s}_{2}}^{V_{1}, V_{2}}(X, A) \subset \bigcup_{\mathbf{s}} \overline{\mathfrak{M}}_{g, k ; \mathbf{s}}^{V}(X, A)
$$

that keep track of contacts with $V_{1}$ and $V_{2}$ separately. There is then a forgetful morphism

$$
f: \overline{\mathfrak{M}}_{g, k ; \mathbf{s}_{1} \mathbf{s}_{2}}^{V_{1}, V_{2}}(X, A) \longrightarrow \overline{\mathfrak{M}}_{g, k ; \mathbf{s}_{1}}^{V_{1}}(X, A)
$$

which drops the relative contacts with $V_{2}$. The total relative evaluation morphisms (1.3) corresponding to the two moduli spaces above are compatible with $f$ and the projection

$$
\pi_{1}:\left(V_{1}\right)_{\mathbf{s}_{1}} \times\left(V_{2}\right)_{\mathbf{s}_{2}} \longrightarrow\left(V_{1}\right)_{\mathbf{s}_{1}},
$$

i.e. the part of the diagram in Figure 1 involving only the corners commutes. The projection $\pi_{1}$ lifts to a smooth map $\widetilde{\pi}_{1}$ between the total spaces of the coverings

$$
\pi_{X ; \mathbf{s}_{1} \mathbf{s}_{2}}^{V_{1}, V_{2}}: \widehat{V}_{X ; \mathbf{s}_{1} \mathbf{s}_{2}} \longrightarrow\left(V_{1}\right)_{\mathbf{s}_{1}} \times\left(V_{2}\right)_{\mathbf{s}_{2}} \text { and } \pi_{X ; \mathbf{s}_{1}}^{V_{1}}:\left(\widehat{V}_{1}\right)_{X ; \mathbf{s}_{1}} \longrightarrow\left(V_{1}\right)_{\mathbf{s}_{1}}
$$

so that the right square in Figure 1 commutes. The relevant base points determining the lifts (1.8) can be chosen so that the resulting lifted evaluation morphisms are compatible with $f$ and $\tilde{\pi}_{1}$, i.e. the left square in Figure 1 commutes.

\subsection{Qualitative applications}

The set of all IP-counts for $(X, V)$ for elements in an orbit for the $\operatorname{Deck}\left(\pi_{X ; \mathbf{s}}^{V}\right)$-action on $H^{*}\left(\widehat{V}_{X ; \mathbf{s}} ; \mathbb{Q}\right)$ depends only on $(X, V, \omega)$, the cohomology class on $X^{k}$, and intrinsic classes on $\overline{\mathfrak{M}}_{g, k ; \mathbf{s}}^{V}(X, A)$, such as descendants. However, the individual IP-counts also depend on the precise choice of the lift (1.8). If $\ell=0$, the cover (1.6) is trivial and these numbers can be indexed by the elements of $\mathcal{R}_{X}^{V}$. This is generally not the case if $\ell \neq 0$, including in the last claim of [8, Lemma 14.5] and in [8, Lemma 14.8]; see [4, Remarks 6.5,6.8]. Because the IP-counts generally depend on the choice of the lift (1.8) and the homology of $\widehat{V}_{X ; \mathbf{s}}$ is usually very complicated, they appear to be of little quantitative use outside of very rare cases. On the other hand, they can sometimes provide qualitative information, as indicated by Theorem 1.1 below. 


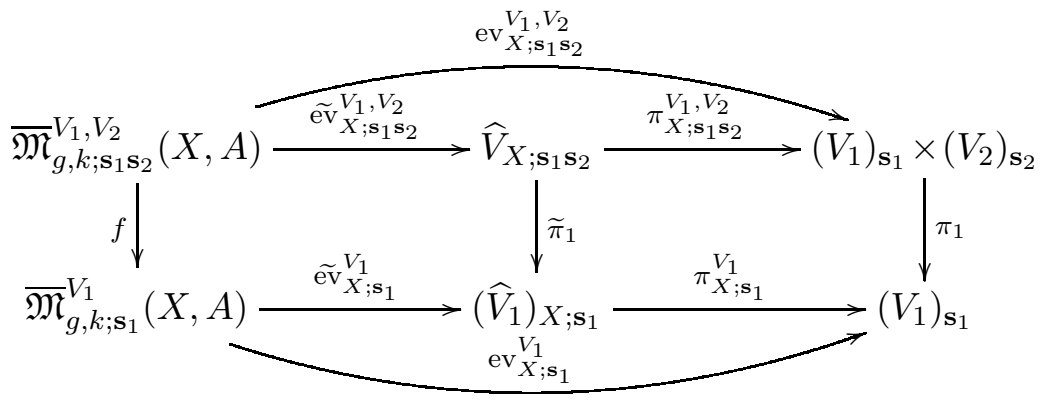

Figure 1: The potential compatibility of the lifted relative evaluation morphisms (1.8).

Theorem 1.1. Let $(X, \omega)$ be a compact symplectic manifold and $V \subset X$ be a compact symplectic divisor which admits a fibration $q: V \longrightarrow\left(S^{1}\right)^{m}$ with a connected fiber $F$ such that $H_{1}(F ; \mathbb{Q})=\{0\}$. If $\ell \in \mathbb{Z}^{+}$and $\mathbf{s} \in \mathbb{Z}_{+}^{\ell}$, then

$$
\operatorname{ev}_{X}^{V *} \alpha \cap\left[\overline{\mathfrak{M}}_{g, k ; \mathbf{s}}^{V}(X, A)\right]^{\mathrm{vir}}=0 \quad \forall \alpha \in H^{r}\left(V_{\mathbf{s}} ; \mathbb{Q}\right), r>\left(\operatorname{dim}_{\mathbb{R}} V\right) \ell-\operatorname{rk}_{\mathbb{Z}} H_{1}(V ; \mathbb{Z})_{X},
$$

i.e. all relative $G W$-invariants of $(X, V, \omega)$ with non-trivial contacts with $V$ and relative insertions $\alpha$ as above vanish.

The proof of this theorem readily extends to disconnected divisors $V$, after replacing the rank of $H_{1}(V ; \mathbb{Z})_{X}$ with the rank of the appropriate submodule of $H_{1}(V ; \mathbb{Z})_{X}$, depending on s; see Remark 6.10.

If $V=\left(S^{1}\right)^{2 n-2}$ and $H^{3}(X ; \mathbb{Z})=0$, then

$$
H_{1}(V ; \mathbb{Z})_{X}=H_{1}(V ; \mathbb{Z}) \approx \mathbb{Z}^{2 n-2} .
$$

In this case, the relative $\mathrm{GW}$-invariants of $(X, V, \omega)$ with non-trivial contacts with $V$ vanish whenever the degree of the relative insertion exceeds $(2 n-2)(\ell-1)$. In particular, the only relative GW-invariants of $(X, V, \omega)$ with a single (but arbitrary order) contact that may be nonzero are those that involve no relative constraint (insertion $1 \in H^{*}(V ; \mathbb{Q})$ ). This particular observation is immediate from (1.7), because $\widehat{V}_{X ;(s)} \approx \mathbb{C}^{n-1}$ and thus $\widehat{V}_{X ;(s)}$ has no positive-degree cohomology for any $s \in \mathbb{Z}^{+}$. We use this fact in [4, Section 6.3] to streamline the proof of [8, (15.4)], after correcting its statement; this formula computes some $\mathrm{GW}$-invariants of the blowup $\widehat{\mathbb{P}}_{9}^{2}$ of $\mathbb{P}^{2}$ at 9 points.

If $V$ is any topological space, a loop of homeomorphisms

$$
\Psi_{t}: V \longrightarrow V, \quad t \in[0,1], \quad \Psi_{0}=\Psi_{1},
$$

and a point $x \in V$ determines a loop $t \longrightarrow \Psi_{t}(x)$ in $V$ and thus an element of $H_{1}(V ; \mathbb{Z})$. The latter is independent of the choice of $x \in V$. We denote the set of all elements of $H_{1}(V ; \mathbb{Z})$ obtained in this way by $\operatorname{Flux}(V)$. It is a subgroup of $H_{1}(V ; \mathbb{Z})$, usually called the flux subgroup (or group). If in addition $V \subset X$ is a compact oriented submanifold of a compact oriented manifold and $H_{1}(V ; \mathbb{Z})_{X}$ is as in (1.5), let

$$
\operatorname{Flux}(V)_{X} \subset H_{1}(V ; \mathbb{Z})_{X}
$$

denote the image of Flux $(V)$ under the quotient projection. 
Theorem 1.2. Let $(X, \omega)$ be a compact symplectic manifold and $V \subset X$ be a compact connected symplectic divisor such that

$$
\operatorname{Flux}(V)_{X}=H_{1}(V ; \mathbb{Z})_{X}
$$

Suppose $A \in H_{2}(X ; \mathbb{Z})$ and $\mathbf{s} \in \mathbb{Z}_{+}^{\ell}$ with $\ell>0$. If $\operatorname{gcd}(\mathbf{s})$ and $\left|\mathcal{R}_{X}^{V}\right|$ are relatively prime, then the IP-counts for $(X, V, \omega)$ in degree $A$ with relative contacts $\mathbf{s}$ are independent of the choice of the lift (1.8) and are thus determined by $(X, V, \omega)$. If in addition

$$
\mathrm{rk}_{\mathbb{Z}} H_{1}(V ; \mathbb{Z})_{X} \in\{0,1\},
$$

then these IP-counts are the same as the corresponding $G W$-invariants of $(X, V, \omega)$.

If the rim tori module $\mathcal{R}_{X}^{V} \approx H_{1}(V ; \mathbb{Z})_{X}$ is infinite, we call $\operatorname{gcd}(\mathbf{s})$ and $\left|\mathcal{R}_{X}^{V}\right|$ relatively prime if $\operatorname{gcd}(\mathbf{s})=1$. Let

$$
H^{*}\left(\widehat{V}_{X ; \mathbf{s}} ; \mathbb{Q}\right)^{\pi_{X ; \mathbf{s}}^{V}}=\left\{\eta \in H^{*}\left(\widehat{V}_{X ; \mathbf{s}} ; \mathbb{Q}\right): g^{*} \eta=\eta \forall g \in \operatorname{Deck}\left(\pi_{X ; \mathbf{s}}^{V}\right)\right\} .
$$

In general, the set of $\mathrm{GW}$-invariants of $(X, V, \omega)$ in degree $A$ with relative contacts $\mathbf{s}$ with all possible cohomology insertions can be identified with the subset of IP-counts with the cohomology insertions in

$$
\pi_{X ; \mathbf{s}}^{V *} H^{*}\left(V_{\mathbf{s}} ; \mathbb{Q}\right) \subset H^{*}\left(\widehat{V}_{X ; \mathbf{s}} ; \mathbb{Q}\right)^{\pi_{X ; \mathbf{s}}^{V}} \subset H^{*}\left(\widehat{V}_{X ; \mathbf{s}} ; \mathbb{Q}\right) ;
$$

the GW-invariants and IP-counts with such insertions are the same by (1.7). The substance of the first conclusion of Theorem 1.2 is that the second inclusion in (1.12) is an equality, as any two lifts (1.8) are related by an element of $\operatorname{Deck}\left(\pi_{X ; \mathbf{s}}^{V}\right)$. The substance of the second conclusion of Theorem 1.2 is that both inclusions in (1.12) are equalities. The cohomology homomorphism $\pi_{X ; \mathrm{s}}^{V *}$ may still not be injective; the $\mathrm{GW}$-invariants of $(X, V, \omega)$ with insertions in its kernel vanish and thus can be disregarded.

Theorem 1.2 is established at the end of Section 6.1. We show that (1.10) implies that the second inclusion in (1.12) is in fact an equality. The first inclusion in (1.12) is an equality if (1.11) holds; see Corollary 5.8. Both inclusions in (1.12) are equalities if $V=\mathbb{T}^{2 n-2}$ and the cover $\widehat{V}_{X ; \text { s }}$ is connected, as can be seen by considering all connected covers of tori. From this observation, we obtain the following conclusion concerning IP-counts relative to tori.

Proposition 1.3. Suppose $(X, \omega)$ is a compact symplectic manifold and $V \subset X$ is a symplectic divisor such that $V \approx \mathbb{T}^{2 n-2}$. Let $A \in H_{2}(X ; \mathbb{Z})$ and $\mathbf{s} \in \mathbb{Z}_{+}^{\ell}$ with $\ell>0$. If $\operatorname{gcd}(\mathbf{s})$ and $\left|\mathcal{R}_{X}^{V}\right|$ are relatively prime, then the $I P$-counts for $(X, V, \omega)$ in degree $A$ with relative contacts $\mathbf{s}$ are the same as the corresponding $G W$-invariants of $(X, V, \omega)$.

Remark 1.4. As pointed out by B. Wieland on MathOverflow, the first inclusion in (1.12) can fail to be an equality as soon as $H_{1}(V ; \mathbb{Z})_{X}$ is at least $\mathbb{Z}^{2}$. It can fail to be an equality even if $\pi_{1}(V)$ is free abelian.

Theorem 1.2 and Proposition 1.3 do not provide any new information about the GW-invariants of $(X, V, \omega)$. However, they can be useful in refining the usual symplectic sum formula in a narrow set of cases. This formula expresses certain sums of GW-invariants of one symplectic manifold in terms of relative GW-invariants of simpler manifolds; Theorem 1.2 and Proposition 1.3 imply that all the summands in each given sum are the same in these cases.

Generalizations of Theorem 1.2 and of Proposition 1.3 to a disconnected divisor $V$ are described in Remark 6.4. 


\subsection{Outline of the paper}

The relevant setting for relative GW-invariants and the symplectic sum formula is the codimension $\mathfrak{c}=2$ case of the topological setup of Section 2, As restricting to the $\mathfrak{c}=2$ case carries no benefit, we consider the general case to the extent possible. Sections 3.1, 4.1, and 4.4 are concerned with changes in the topology of manifolds under surgery that are directly relevant in the symplectic sum context. The rim tori module $\mathcal{R}_{X}^{V}$ and the vanishing cycles module $\mathcal{R}_{X, Y}^{V}$ are described explicitly in Sections 3.2 and 4.2 , respectively, with the aim of easily computing them in many situations. Section 4.3 compares the rim tori modules before and after surgery. The notation for the abelian covers relevant for our purposes is introduced in Section 5.1 some of their properties, focusing on finite generation of the (co)homology, are discussed in Section 5.2. In Section 6.1, we define the intended rim tori coverings (1.6) of [7, Section 5] as special cases of the abelian covers of Section 5.1, show that the evaluation morphisms (1.3) lift to these covers, and establish Theorem 1.2. In Section 6.2, we show that these lifts can be chosen systematically, in respect to the intended applications in [8] and the diagram in Figure 1, see Theorem 6.5. Theorem 1.1 is established in Section 6.3.

The main purpose of this paper is to investigate the topological aspects of the rim tori refinement to the standard relative GW-invariants in preparation for considering its applicability in the symplectic sum context in [4. We pre-suppose a suitable analytic framework for the construction of relative GW-invariants and describe the necessary steps to implement the idea of [7, Section 5] as an enhancement on existing constructions. We deduce some qualitative applications arising from this refinement and discuss its usability for quantitative purposes. A significant number of examples are included for illustrative purposes.

The authors would like to thank E. Ionel, D. McDuff, M. McLean, J. Milnor, D. Ruberman, J. Starr, M. Wendt, and B. Wieland for enlightening discussions.

\section{General topological context}

The symplectic sum construction is a surgery operation that cuts out tubular neighborhoods of a common submanifold from two manifolds and glues the remainders along the boundaries of the two tubular neighborhoods. Below we discuss central topological aspects of this construction from a more general perspective. Throughout this paper, by a manifold we will mean a smooth manifold.

\subsection{Complement of submanifold}

Let $X$ be an oriented manifold, $V \subset X$ be a compact oriented submanifold of codimension $\mathfrak{c}$, $S_{X} V \subset \mathcal{N}_{X} V$ be the sphere subbundle of the normal bundle of $V$ in $X$, and

$$
\mathcal{R}_{X}^{V} \equiv \operatorname{ker}\left\{\iota_{X-V *}^{X}: H_{\mathfrak{c}}(X-V ; \mathbb{Z}) \longrightarrow H_{\mathfrak{c}}(X ; \mathbb{Z})\right\} .
$$

By Lemma 3.1, each element of $\mathcal{R}_{X}^{V}$ can be represented by a cycle of the form $\iota_{S_{X} V}^{X-V}\left(\left.S_{X} V\right|_{\gamma}\right)$ for some loop $\gamma \subset V$; see the end of Section 3.1. In the $\mathfrak{c}=2$ case, i.e. as in (1.4), these cycles are called rim tori in [7, 8]. 
Suppose in addition that $f: Z \longrightarrow X$ is an $\mathfrak{c}$-pseudocycle, as in [21, Section 1.1], and $x \in f^{-1}(V)$ is an isolated point. We define the order of contact of $f$ with $V$ at $x, \operatorname{ord}_{x}^{V} f \in \mathbb{Z}$, as follows. On a small neighborhood of $x, f$ can be homotoped without changing its intersection with $V$ so that it takes a small sphere $S_{Z} x$ in $T_{x} Z$ to a small sphere $\left.S_{X} V\right|_{f(x)} \subset \mathcal{N}_{V} X$; the number ord ${ }_{x}^{V} f$ is the degree of this map. This definition agrees with the definition used in the construction of $\overline{\mathfrak{M}}_{g, k ; \mathbf{s}}^{V}(X, A)$.

We now combine two pseudocycles with the same contacts with $V$ into a pseudocycle to $X-V$. Suppose

$$
f:\left(Z, x_{1}, \ldots, x_{\ell}\right) \longrightarrow(X, V) \quad \text { and } \quad f^{\prime}:\left(Z^{\prime}, x_{1}^{\prime}, \ldots, x_{\ell}^{\prime}\right) \longrightarrow(X, V)
$$

are two c-pseudocycles such that

$$
\begin{gathered}
f^{-1}(V)=\left\{x_{1}, \ldots, x_{\ell}\right\}, \quad f^{\prime-1}(V)=\left\{x_{1}^{\prime}, \ldots, x_{\ell}^{\prime}\right\}, \\
f\left(x_{i}\right)=f^{\prime}\left(x_{i}^{\prime}\right), \quad \operatorname{ord}_{x_{i}}^{V} f=\operatorname{ord}_{x_{i}^{\prime}}^{V} f^{\prime} \quad \forall i=1,2, \ldots, \ell .
\end{gathered}
$$

We can then obtain a smooth map $f \#\left(-f^{\prime}\right): Z \# Z^{\prime} \longrightarrow X-V$ by

- removing small balls $B_{x_{i}}$ and $B_{x_{i}^{\prime}}$ around each of the points $x_{i}$ and $x_{i}^{\prime}$ to form manifolds with boundary $\hat{Z}$ and $\hat{Z}^{\prime}$,

- forming a smooth oriented manifold $Z \#\left(-Z^{\prime}\right)$ by identifying the $i$-th boundary components of $\hat{Z}$ and $\hat{Z}^{\prime}$ by an orientation-preserving diffeomorphism $\varphi_{i}:(\partial \hat{Z})_{i} \longrightarrow\left(\partial \hat{Z}^{\prime}\right)_{i}$ for each $i$,

- homotoping $f$ and $f^{\prime}$ on small neighborhoods of $\partial B_{x_{i}}$ and $\partial B_{x_{i}^{\prime}}$ within a small ball around $f\left(x_{i}\right)=f^{\prime}\left(x_{i}^{\prime}\right)$ in $X$ so that $f=f^{\prime} \circ \varphi_{i}$ for all $i$.

The last condition is achievable because the degrees of $f, f^{\prime} \circ \varphi_{i}: S^{\mathfrak{c}-1} \longrightarrow S^{\mathfrak{c}-1}$ are the same and the degree homomorphism $\pi_{\mathfrak{c}-1}\left(S^{\mathfrak{c}-1}\right) \longrightarrow \mathbb{Z}$ is an isomorphism if $\mathfrak{c} \geq 2$.

The above construction of $f \#\left(-f^{\prime}\right)$ depends only on $f, f^{\prime}$, and choices of degree 1 maps from $\ell$ disjoint copies of $[0,1] \times S^{\mathfrak{c}-1}$ to $[0,1] \times S^{\mathfrak{c}-1}$. Thus, $f$ and $f^{\prime}$ completely determine the homology class of $f \#\left(-f^{\prime}\right)$. If in addition $[f]=\left[f^{\prime}\right]$ in $H_{\mathfrak{c}}(X ; \mathbb{Z})$, then $\left[f \#\left(-f^{\prime}\right)\right] \in \mathcal{R}_{X}^{V}$.

\subsection{Splice of two manifolds}

If $X$ and $Y$ are manifolds, $V \subset X, Y$ is a closed submanifold, and $\varphi: S_{X} V \longrightarrow S_{Y} V$ is a diffeomorphism commuting with the projections to $V$, let $X \#_{\varphi} Y$ be the manifold obtained by gluing the complements of tubular neighborhoods of $V$ in $X$ and $Y$ by $\varphi$ along their common boundary. If $X$ and $Y$ are oriented and $\varphi$ is orientation-reversing, then $X \#_{\varphi} Y$ is oriented as well.

We denote by

$$
q_{\varphi}: X \#_{\varphi} Y \longrightarrow X \cup_{V} Y
$$

a continuous map which restricts to the identity outside of a tubular neighborhood of $S_{X} V={ }_{\varphi} S_{Y} V$, is a diffeomorphism on the complement of $q_{\varphi}^{-1}(V)$, and restricts to the bundle projection $S_{X} V \longrightarrow V$. We will call such a map $q_{\varphi}$ a collapsing map. If $\mathfrak{c}$ is the codimension of $V$ in $X$ and $Y$, let

$$
\mathcal{R}_{X, Y}^{V} \equiv \operatorname{ker}\left\{q_{\varphi_{*}}: H_{\mathfrak{c}}\left(X \#{ }_{\varphi} Y ; \mathbb{Z}\right) \longrightarrow H_{\mathfrak{c}}\left(X \cup_{V} Y ; \mathbb{Z}\right)\right\}
$$


By the $m=\mathfrak{c}$ case of Lemma 4.1, this collection of vanishing cycles is the span of the images of $\mathcal{R}_{X}^{V}$ and $\mathcal{R}_{Y}^{V}$ under the homology homomorphisms induced by the inclusions

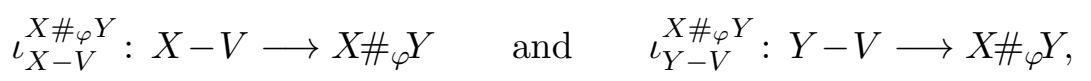

respectively.

Suppose in addition $X, Y$, and $V$ are compact and oriented and $V_{1}, \ldots, V_{N}$ are the topological components of $V$. Let

$$
\begin{aligned}
H_{\mathfrak{c}}(X ; \mathbb{Z}) \times_{V} H_{\mathfrak{c}}(Y ; \mathbb{Z})=\left\{\left(A_{X}, A_{Y}\right) \in H_{\mathfrak{c}}(X ; \mathbb{Z}) \times H_{\mathfrak{c}}(Y ; \mathbb{Z}):\right. \\
\left.A_{X} \cdot X V_{r}=A_{Y \cdot Y} V_{r} \quad \forall r=1, \ldots, N\right\},
\end{aligned}
$$

where $\cdot_{X}$ and $\cdot_{Y}$ denote the homology intersection pairings in $X$ and $Y$, respectively. Given an orientation-reversing diffeomorphism $\varphi$, we describe below an operation gluing $\mathfrak{c}$-cycles in $X$ and $Y$ into $\mathfrak{c}$-cycles in $X \#_{\varphi} Y$. It induces a homomorphism

$$
H_{\mathfrak{c}}(X ; \mathbb{Z}) \times_{V} H_{\mathfrak{c}}(Y ; \mathbb{Z}) \longrightarrow H_{\mathfrak{c}}\left(X \#{ }_{\varphi} Y ; \mathbb{Z}\right) / \mathcal{R}_{X, Y}^{V}, \quad\left(A_{X}, A_{Y}\right) \longrightarrow A_{X} \#_{\varphi} A_{Y},
$$

which is central to the symplectic sum formula for GW-invariants.

Suppose

$$
f_{X}:\left(Z_{X}, x_{1}, \ldots, x_{\ell}\right) \longrightarrow(X, V) \quad \text { and } \quad f_{Y}:\left(Z_{Y}, y_{1}, \ldots, y_{\ell}\right) \longrightarrow(Y, V)
$$

are $\mathfrak{c}$-pseudocycles with boundary disjoint from $V$ such that

$$
\begin{gathered}
f_{X}^{-1}(V)=\left\{x_{1}, \ldots, x_{\ell}\right\}, \quad f_{Y}^{-1}(V)=\left\{y_{1}, \ldots, y_{\ell}\right\}, \\
f_{X}\left(x_{i}\right)=f_{Y}\left(y_{i}\right), \quad \operatorname{ord}_{x_{i}}^{V} f_{X}=\operatorname{ord}_{y_{i}}^{V} f_{Y} \quad \forall i=1,2, \ldots, \ell .
\end{gathered}
$$

We can then obtain a smooth map $f_{X} \#_{\varphi} f_{Y}: Z_{X} \# Z_{Y} \longrightarrow X \#_{\varphi} Y$ by

- removing small balls $B_{x_{i}}$ and $B_{y_{i}}$ around each of the points $x_{i}$ and $y_{i}$ to form manifolds with boundary $\hat{Z}_{X}$ and $\hat{Z}_{Y}$,

- forming a smooth oriented manifold $Z_{X} \# Z_{Y}$ by identifying the $i$-th boundary components of $\hat{Z}_{X}$ and $\hat{Z}_{Y}$ by an orientation-reversing diffeomorphism $\varphi_{i}:\left(\partial \hat{Z}_{X}\right)_{i} \longrightarrow\left(\partial \hat{Z}_{Y}\right)_{i}$ for each $i$,

- homotoping $f_{X}$ and $f_{Y}$ on small neighborhoods of $\partial B_{x_{i}}$ and $\partial B_{y_{i}}$ within small balls around $f_{X}\left(x_{i}\right)$ in $X$ and $f_{Y}\left(y_{i}\right)$ in $Y$ so that $\varphi \circ f_{X}=f_{Y} \circ \varphi_{i}$ for all $i$.

The last condition is achievable because the degrees of

$$
\varphi \circ f_{X} \circ \varphi_{i}^{-1}, f_{Y}: S^{\mathfrak{c}-1} \longrightarrow S^{\mathfrak{c}-1}
$$

are the same.

The above construction of $f_{X} \#_{\varphi} f_{Y}$ depends only on $f_{X}, f_{Y}$, and choices of degree -1 maps from $\ell$ disjoint copies of $[0,1] \times S^{\mathfrak{c}-1}$ to $[0,1] \times S^{\mathfrak{c}-1}$. Thus, $f_{X}$ and $f_{Y}$ completely determine the homology class of $f_{X} \#_{\varphi} f_{Y}$. Furthermore,

$$
\left.q_{\varphi *}\left(\left[f_{X} \#_{\varphi} f_{Y}\right]\right)=\iota_{X *}^{X \cup_{V} Y}\left(\left[f_{X}\right)\right]\right)+\iota_{Y *}^{X \cup_{V} Y}\left(\left[f_{Y}\right]\right) \in H_{\mathfrak{c}}\left(X \cup_{\varphi} Y ; \mathbb{Z}\right) .
$$


If $\left[f_{X}\right]=\left[f_{X}^{\prime}\right]$ in $H_{\mathfrak{c}}(X ; \mathbb{Z}),\left[f_{X} \#\left(-f_{X}^{\prime}\right)\right] \in \mathcal{R}_{X}^{V}$ by Section 2.1, Thus, the homology class of $f_{X} \#_{\varphi} f_{Y}$ in $X \#_{\varphi} Y$ as above is determined by the homology classes of $f_{X}$ in $X$ and $f_{Y}$ in $Y$ only up to an element of $\mathcal{R}_{X, Y}^{V}$.

Suppose $\left(X, \omega_{X}\right)$ and $\left(Y, \omega_{Y}\right)$ are symplectic manifolds with a common compact symplectic divisor $V \subset X, Y$ such that

$$
e\left(\mathcal{N}_{X} V\right)=-e\left(\mathcal{N}_{Y} V\right) \in H^{2}(V ; \mathbb{Z}) .
$$

The symplectic sum construction of [6, 18] then produces a symplectic manifold $\left(X \#{ }_{V} Y, \omega_{\#}\right)$ of the form $X \#_{\varphi} Y$. Let $\eta$ be a coset of $H_{2}\left(X \#_{V} Y ; \mathbb{Z}\right)$ modulo $\mathcal{R}_{X, Y}^{V}$. According to the symplectic sum formulas of [11, 10], the sum of the genus $g \mathrm{GW}$-invariants of $X \#_{V} Y$ in degrees $A \in \eta$ is the same as the sum of the genus $g \mathrm{GW}$-invariants of $\left(X, V, \omega_{X}\right)$ and $\left(Y, V, \omega_{Y}\right)$ of degrees $A_{X}$ and $A_{Y}$ such that $A_{X} \#_{\varphi} A_{Y}=\eta$. It would of course be preferable to express individual GW-invariants of $X \#_{V} Y$ in terms of relative $\mathrm{GW}$-invariants of $\left(X, V, \omega_{X}\right)$ and $\left(Y, V, \omega_{Y}\right)$. The rim tori refinement of standard relative invariants is suggested in [7] with the aim of resolving this deficiency in [8]. In [4, we discuss to what extent this is achieved.

\section{Cutting out a submanifold}

We discuss changes in the homology after cutting out a submanifold in Section 3.1, Lemma 3.1 contains [7, Lemma 5.2] and the corresponding part of the proof of the former is essentially the same as the proof of the latter. We use it in Section 3.2 to give an explicit description of the rim tori module $\mathcal{R}_{X}^{V}$ and to compare it with the rim tori module $\mathcal{R}_{X}^{U \cup V}$ for a submanifold with additional connected components.

\subsection{Changes in homology}

Given a manifold $X$ and a closed submanifold $V \subset X$, we will view the sphere subbundle $S_{X} V$ of the normal bundle $\mathcal{N}_{X} V$ of $V$ in $X$ as a hypersurface in $X$. If in addition $V$ is compact oriented and the codimension of $V$ in $X$ is $\mathfrak{c}$, we define

$$
\Delta_{X}^{V}: H_{m}(V ; \mathbb{Z}) \longrightarrow H_{m+\mathfrak{c}-1}\left(S_{X} V ; \mathbb{Z}\right), \quad \Delta_{X}^{V}(\gamma)=\operatorname{PD}_{S_{X} V}\left(q_{X}^{V^{*}}\left(\mathrm{PD}_{V} \gamma\right)\right),
$$

where $q_{X}^{V}: S_{X} V \longrightarrow V$ is the projection map. If $X$ is also compact, let

$$
\cap V: H_{*}(X ; \mathbb{Z}) \longrightarrow H_{*-\mathfrak{c}}(V ; \mathbb{Z}), \quad A \cap V=\mathrm{PD}_{V}\left(\left.\left(\mathrm{PD}_{X} A\right)\right|_{V}\right) .
$$

If $U \subset X-V$ is another subset (possibly empty), we take

$$
\cap V: H_{*}(X-U ; \mathbb{Z}) \stackrel{\iota_{X}^{X}-U *}{\longrightarrow} H_{*}(X ; \mathbb{Z}) \stackrel{\cap V}{\longrightarrow} H_{*-\mathfrak{c}}(V ; \mathbb{Z})
$$

to be the composition. Let

$$
H_{X-U}^{V}=\left\{A \cap V: A \in H_{\mathfrak{c}+1}(X-U ; \mathbb{Z})\right\} \subset H_{1}(V ; \mathbb{Z}), \quad H_{1}(V ; \mathbb{Z})_{X-U}=\frac{H_{1}(V ; \mathbb{Z})}{H_{X-U}^{V}}
$$


Lemma 3.1. Suppose $X$ is a compact oriented manifold, $V \subset X$ is a compact oriented submanifold of codimension $\mathfrak{c}$, and $U \subset X-V$ is a compact subset. Then, the sequence

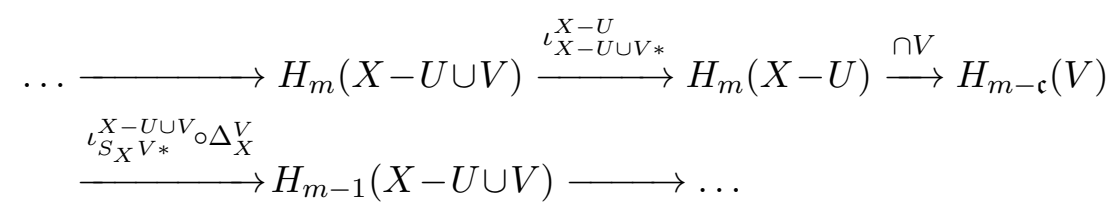

is exact for any coefficient ring.

Proof. Taking the Poincare dual of the Gysin sequence for $S_{X} V \longrightarrow V$, we obtain an exact sequence

$$
\ldots \stackrel{\Delta_{X}^{V}}{\longrightarrow} H_{m}\left(S_{X} V\right) \stackrel{q_{X *}^{V}}{\longrightarrow} H_{m}(V) \stackrel{e\left(\mathcal{N}_{X} V\right) \cap}{\longrightarrow} H_{m-\mathfrak{c}}(V) \stackrel{\Delta_{X}^{V}}{\longrightarrow} H_{m-1}\left(S_{X} V\right) \stackrel{q_{X *}^{V}}{\longrightarrow} \ldots
$$

By the proof of Mayer-Vietoris for $X-U=(X-U \cup V) \cup_{S_{X} V} V$,

$$
\begin{aligned}
& \ldots \stackrel{\delta_{X}}{\longrightarrow} H_{m}\left(S_{X} V\right) \stackrel{\left.\stackrel{\left(\iota_{S_{X} V *}^{X}-U \cup V\right.}{V *}, q_{X *}^{V}\right)}{\longrightarrow} H_{m}(X-U \cup V) \oplus H_{m}(V) \stackrel{\stackrel{\iota_{X-U \cup V *}^{X-U}+\iota_{V *}^{X-U}}{\longrightarrow} H_{m}(X-U)}{\stackrel{\delta_{X}}{\longrightarrow} H_{m-1}\left(S_{X} V\right) \stackrel{\left(\iota_{S_{X} V *}^{X-U \cup V},-q_{X *}^{V}\right)}{\longrightarrow} \ldots,}
\end{aligned}
$$

the connecting homomorphism $\delta_{X}$ is the composition $\Delta_{X}^{V} \circ(\cdot \cap V)$, at least up to sign. Since

$$
\iota_{X-U \cup V *}^{X-U}(A) \cap V=0 \quad \forall A \in H_{m}(X-U \cup V),
$$

the claim now follows from the observation that

$$
\iota_{V^{*}}^{X-U}(A) \cap V=e\left(\mathcal{N}_{X} V\right) \cap A \quad \forall A \in H_{m}(V),
$$

at least up to sign (dependent on one's definitions of cup and cap products and Poincare dual); see below for details.

The composition of the first two labeled homomorphisms in (3.3) being 0 is equivalent to (3.6). If $A_{X-U} \cap V=0$ for some $A_{X-U} \in H_{m}(X-U)$, then $\delta_{X}\left(A_{X-U}\right)=0$ and so

$$
A_{X-U}=\iota_{X-U \cup V *}^{X-U}\left(A_{X-U \cup V}\right)+\iota_{V *}^{X-U}\left(A_{V}\right) \text { for some } A_{X-U \cup V} \in H_{m}(X-U \cup V), A_{V} \in H_{m}(V),
$$

by the exactness of (3.5). By $A_{X-U} \cap V=0$, (3.6), and (3.7), $e\left(\mathcal{N}_{X} V\right) \cap A_{V}=0$ and so

$$
A_{V}=q_{X *}^{V}\left(A_{S_{X} V}\right) \quad \text { for some } A_{S_{X} V} \in H_{m}\left(S_{X} V\right)
$$

by the exactness of (3.4). By the last two displayed expressions,

$$
A_{X-U}=\iota_{X-U \cup V *}^{X-U}\left(A_{X-U \cup V}+\iota_{S_{X} V *}^{X-U \cup V}\left(A_{S_{X} V}\right)\right),
$$

which establishes the exactness of (3.3) at $H_{m}(X-U)$.

The composition of the last two labeled homomorphisms in (3.3) being 0 follows from the exactness of (3.5) and $\delta_{X}=\Delta_{X}^{V} \circ(\cdot \cap V)$. Suppose

$$
\left\{\iota_{S_{X} V *}^{X-U \cup V} \circ \Delta_{X}^{V}\right\}\left(A_{V}\right)=0 \text { for some } A_{V} \in H_{m-\mathfrak{c}}(V) .
$$


Since $q_{X *}^{V} \circ \Delta_{X}^{V}=0$ by the exactness of (13.4) ,

$$
\Delta_{X}^{V}\left(A_{V}\right)=\delta_{X}\left(A_{X-U}\right)=\Delta_{X}^{V}\left(A_{X-U} \cap V\right) \text { for some } A_{X-U} \in H_{m}(X-U)
$$

by the exactness of (3.5). Thus,

$$
A_{V}=A_{X-U} \cap V+e\left(\mathcal{N}_{X} V\right) \cap A_{V}^{\prime}=\left(A_{X-U}+\iota_{V *}^{X-U}\left(A_{V}^{\prime}\right)\right) \cap V \quad \text { for some } A_{V}^{\prime} \in H_{m}(V)
$$

by the exactness of (3.4) and (3.7). This establishes the exactness of (3.3) at $H_{m-\mathfrak{c}}(V)$.

The vanishing of the composition

$$
\iota_{X-U \cup V *}^{X-U} \circ\left\{\iota_{S_{X} V *}^{X-U \cup V} \circ \Delta_{X}^{V}\right\}: H_{m-\mathfrak{c}}(V) \longrightarrow H_{m-1}(X-U)
$$

follows from the exactness of (3.5). If $\iota_{X-U \cup V *}^{X-U}\left(A_{X-U \cup V}\right)=0$ for some $A_{X-U \cup V} \in H_{m-1}(X-U \cup V)$, then

$$
A_{X-U \cup V}=\iota_{S_{X} V *}^{X-U \cup V}\left(A_{S_{X} V}\right), \quad q_{X *}^{V}\left(A_{S_{X} V}\right)=0 \quad \text { for some } A_{S_{X} V} \in H_{m-1}\left(S_{X} V\right)
$$

by the exactness of (3.5). By the exactness of (3.4) and $q_{X *}^{V}\left(A_{S_{X} V}\right)=0$,

$$
A_{S_{X} V}=\Delta_{X}^{V}\left(A_{V}\right) \text { for some } A_{V} \in H_{m-\mathfrak{c}}(V) \text {. }
$$

This establishes the exactness of (3.3) at $H_{m-1}(X-U \cup V)$.

By [21, Theorem 1.1], every integral homology class in a manifold can be represented by a pseudocycle. If $V$ is as in (3.1) and $f: Z \longrightarrow V$ is a pseudocycle, then the pseudocycle

$$
\pi_{2}: f^{*} S_{X} V \equiv\left\{(z, v) \in Z \times S_{X} V: f(z)=q_{X}^{V}(v)\right\} \longrightarrow S_{X} V
$$

where $\pi_{2}: Z \times S_{X} V \longrightarrow S_{X} V$ is the projection on the second coordinate, represents $\Delta_{X}^{V}([f])$. If $Z=S^{1}, f^{*} S_{X} V \longrightarrow S^{1}$ is a trivial $S^{\mathfrak{c}-1}$-bundle and thus $f$ lifts to a map

$$
\tilde{f}: S^{1} \times S^{\mathfrak{c}-1} \longrightarrow\left\{q_{X}^{V}\right\}^{-1}\left(f\left(S^{1}\right)\right) \subset S_{X} V \quad \text { s.t. } \quad q_{X}^{V} \circ \tilde{f}=f \circ \pi_{1},
$$

where $\pi_{1}: S^{1} \times S^{\mathfrak{c}-1} \longrightarrow S^{1}$ is the projection on the first component. Thus, the elements of the module $\mathcal{R}_{X-U}^{V}$ as in (2.1) can be represented by cycles of the form $\iota_{S_{X} V}^{X-U U V}\left(\left.S_{X} V\right|_{\gamma}\right)$ for loops $\gamma \subset V$, according to Lemma 3.1 .

\subsection{The rim tori}

We now use Lemma 3.1 to describe the rim tori module $\mathcal{R}_{X-U}^{V}$ explicitly and to compare it with other such modules. The first corollary below is an immediate consequence of the $m=\mathfrak{c}$ case of this lemma.

Corollary 3.2. Suppose $X$ is a compact oriented $n$-manifold, $V \subset X$ is a compact oriented submanifold of codimension $\mathfrak{c}$, and $U \subset X-V$ is a compact subset. Then, the induced homomorphism

$$
\iota_{S_{X} V *}^{X-U \cup V} \circ \Delta_{X}^{V}: H_{1}(V ; \mathbb{Z})_{X-U} \longrightarrow \mathcal{R}_{X-U}^{V} \subset H_{\mathfrak{c}}(X-U \cup V ; \mathbb{Z})
$$

is well-defined and is an isomorphism. In particular, if the restriction homomorphism

$$
H^{n-\mathfrak{c}-1}(X ; \mathbb{Z}) \longrightarrow H^{n-\mathfrak{c}-1}(V ; \mathbb{Z}),
$$

is zero, then $\mathcal{R}_{X-U}^{V} \approx H_{1}(V ; \mathbb{Z})$. 


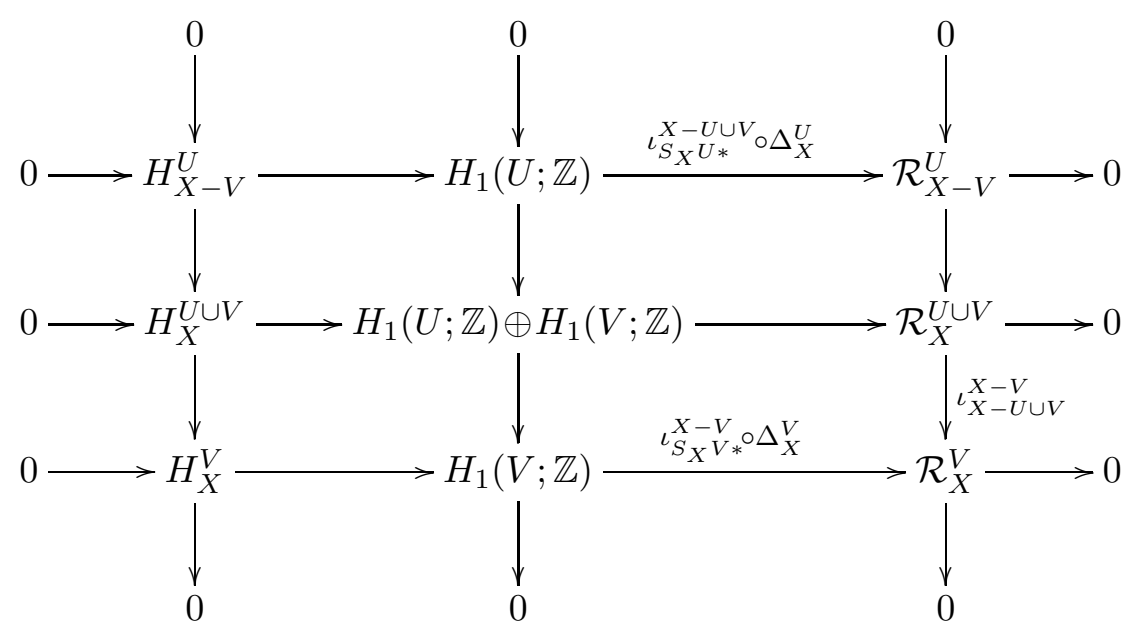

Figure 2: Comparison of rim tori.

Corollary 3.3. Suppose $X$ is a compact oriented manifold and $U, V \subset X$ are compact oriented disjoint submanifolds of codimension $\mathfrak{c}$. Then, the homology homomorphisms induced by inclusions give rise to the commutative square of short exact sequences of Figure 国.

Proof. The rows in this diagram are exact by Corollary 3.2. The exactness of the middle column is clear, as is the exactness of the last column at $\mathcal{R}_{X-V}^{U}$. The last column is exact at $\mathcal{R}_{X}^{U U V}$ by (2.1), with $(X, V)$ replaced by $(X-V, U)$. By the exactness of (3.5),

$$
\begin{aligned}
& \mathcal{R}_{X}^{V}=\left\{\iota_{S_{X} V^{*}}^{X-V}\left(A_{S_{X} V}\right): A_{S_{X} V} \in H_{\mathfrak{c}}\left(S_{X} V ; \mathbb{Z}\right), q_{X *}^{V}\left(S_{X} V\right)=0\right\}, \\
& \mathcal{R}_{X}^{U \cup V} \supset\left\{\iota_{S_{X} V *}^{X-U \cup V}\left(A_{S_{X} V}\right): A_{S_{X} V} \in H_{\mathfrak{c}}\left(S_{X} V ; \mathbb{Z}\right), q_{X *}^{V}\left(S_{X} V\right)=0\right\} .
\end{aligned}
$$

This implies that the last column is exact at $\mathcal{R}_{X}^{V}$.

By (3.2) and (3.6) with $U=\emptyset$,

$$
H_{X-V}^{U} \oplus 0 \subset H_{X}^{U \cup V} \cap H_{1}(U ; \mathbb{Z}) \oplus 0=\left\{(A \cap U, 0): A \in H_{\mathfrak{c}+1}(X ; \mathbb{Z}) \text { s.t. } A \cap V=0\right\} .
$$

In particular, the left column in the diagram is exact at $H_{X-V}^{U}$. By the exactness of (3.3) with $m=\mathfrak{c}+1$ and $U=\emptyset$ at the second position, the above inclusion is in fact an equality and the left column is exact at $H_{X}^{U U V}$. The exactness of the left column at $H_{X}^{U U V}$ is immediate from (3.2).

The commutativity of the bottom right square is equivalent to the homomorphism

$$
\iota_{X-U \cup V *}^{X-V} \circ \iota_{S_{X} U *}^{X-U \cup V} \circ \Delta_{X}^{U}: H_{1}(U ; \mathbb{Z}) \longrightarrow H_{\mathfrak{c}}(X-V ; \mathbb{Z})
$$

being zero. This is immediate from the exactness of (3.5) with $U$ and $V$ interchanged, since $q_{X *}^{U} \circ \Delta_{X}^{U}=0$ by the exactness of (3.4). The commutativity of the other three squares is clear. 
Example 3.4. Let $Z$ be a compact oriented manifold and $E_{1}, E_{2} \longrightarrow Z$ be complex vector bundles of ranks $r_{1}$ and $r_{2}$, respectively. By [19, Theorem 5.7.9], there is a commutative diagram

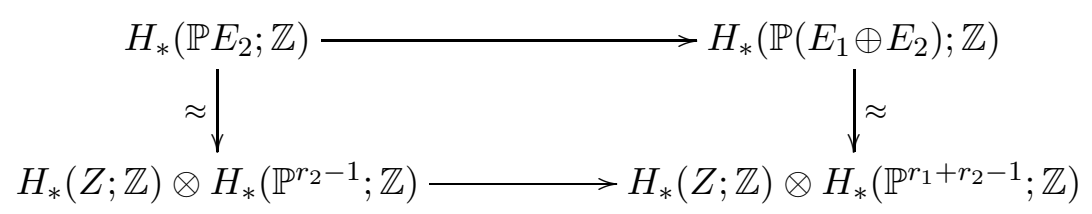

of homomorphisms of modules. In particular, the homomorphism

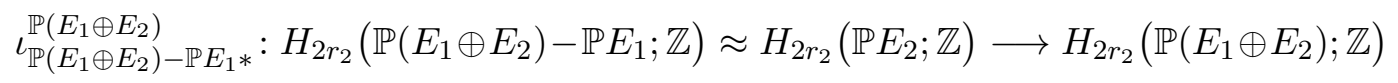

is injective. Thus,

$$
\mathcal{R}_{\mathbb{P}\left(E_{1} \oplus E_{2}\right)}^{\mathbb{P} E_{1}}=\{0\} .
$$

In the case $r_{2}=1$, which is the most relevant for our purposes, this statement follows from $\mathbb{P} E_{2}$ being a section of the fiber bundle $\mathbb{P}\left(E_{1} \oplus E_{2}\right) \longrightarrow Z$.

Example 3.5. Let $\widehat{\mathbb{P}}_{9}^{2}$ denote the blowup of $\mathbb{P}^{2}$ at the 9 intersection points of a general pair of smooth cubic curves $C_{1}$ and $C_{2}$, i.e. the base locus of a general pencil of cubics in $\mathbb{P}^{2}$. The proper transforms of these cubics in $\widehat{\mathbb{P}}_{9}^{2}$ are pairwise disjoint and form a fibration $\pi: \widehat{\mathbb{P}}_{9}^{2} \longrightarrow \mathbb{P}^{1}$, obtained by sending each point in $\widehat{\mathbb{P}}_{9}^{2}$ to the cubic in the pencil passing through it. A smooth fiber $F$ of $\pi$ is a torus $\mathbb{T}^{2}$; there are also 12 singular fibers, each of which is a sphere with a transverse self-intersection. By Corollary 3.2 ,

$$
\mathcal{R}_{\mathbb{P}_{9}^{2}}^{F} \approx H_{1}(F ; \mathbb{Z}) \approx \mathbb{Z}^{2}
$$

because $H^{1}\left(\widehat{\mathbb{P}}_{9}^{2} ; \mathbb{Z}\right)=0$.

Example 3.6. Let $F$ be a compact oriented manifold, $X=\mathbb{P}^{1} \times F, F_{0}=\{0\} \times F$, and $F_{\infty}=\{\infty\} \times F$. Then,

$$
\begin{aligned}
H_{X}^{F_{0}}=H_{1}\left(F_{0} ; \mathbb{Z}\right) & =H_{1}(F ; \mathbb{Z}), \quad H_{X-F_{0}}^{F_{\infty}}=\{0\} \subset H_{1}\left(F_{\infty} ; \mathbb{Z}\right)=H_{1}(F ; \mathbb{Z}), \\
H_{X}^{F_{0} \cup F_{\infty}} & =H_{\Delta} \subset H_{1}\left(F_{0} \cup F_{\infty} ; \mathbb{Z}\right)=H_{1}(F ; \mathbb{Z}) \oplus H_{1}(F ; \mathbb{Z}),
\end{aligned}
$$

where $H_{\Delta}$ is the diagonal subgroup. By Lemma 3.1 or Corollary 3.2 , the homomorphism

$$
\iota_{S_{X} F_{0} *}^{X-F_{0} \cup F_{\infty}} \circ \Delta_{X}^{F_{0}}: H_{1}\left(F_{0} ; \mathbb{Z}\right)=H_{1}(F ; \mathbb{Z}) \longrightarrow \mathcal{R}_{X}^{F_{0} \cup F_{\infty}} \subset H_{\mathfrak{c}}\left(X-F_{0} \cup F_{\infty} ; \mathbb{Z}\right)
$$

is an isomorphism. Under this identification, the last labeled homomorphism in (3.3) with $m=3$ corresponds to

$$
H_{1}\left(F_{0} \cup F_{\infty} ; \mathbb{Z}\right)=H_{1}(F ; \mathbb{Z}) \oplus H_{1}(F ; \mathbb{Z}) \longrightarrow H_{1}(F ; \mathbb{Z}), \quad\left(\gamma_{0}, \gamma_{\infty}\right) \longrightarrow \gamma_{0}-\gamma_{\infty}
$$

\section{Gluing along a common submanifold}

We discuss changes in the homology and cohomology after gluing two manifolds along a common submanifold in Sections 4.1 and 4.4, respectively. We use Lemma 4.1 in Section 4.2 to express the vanishing cycles module $\mathcal{R}_{X, Y}^{V}$ defined in (2.2) in terms of the rim tori modules $\mathcal{R}_{X}^{V}$ and $\mathcal{R}_{Y}^{V}$ defined in (2.1). This lemma is used in Section 4.3 to compare the rim tori modules before and after gluing. Lemma 4.11 contains the precise statements of (a) and (b) at the bottom of [8, p996]; it is useful for determining the cohomology insertions compatible with the symplectic sum formula for GW-invariants. 


\subsection{Changes in homology}

Continuing with the notation introduced in Section 2.2, we relate the analogue of the module (2.2) for homology of any dimension to such analogues of the modules (2.1) for the two pieces.

Lemma 4.1. If $X$ and $Y$ are manifolds, $V \subset X, Y$ is a closed submanifold, $\varphi: S_{X} V \longrightarrow S_{Y} V$ is a diffeomorphism commuting with the projections to $V$, and $q_{\varphi}: X \#_{\varphi} Y \longrightarrow X \cup_{V} Y$ is a collapsing map, then

$$
\begin{aligned}
& \operatorname{ker}\left\{q_{\varphi *}: H_{m}\left(X \#_{\varphi} Y\right) \longrightarrow H_{m}\left(X \cup_{\varphi} Y\right)\right\} \\
& =\left\{\begin{array}{c}
X \#_{\varphi} Y \\
\iota_{X-V *}
\end{array}\left(A_{X-V}\right): A_{X-V} \in H_{m}(X-V), \iota_{X-V *}^{X}\left(A_{X-V}\right)=0\right\} \\
& =\left\{\iota_{Y-V *}^{X \#_{\varphi} Y}\left(A_{Y-V}\right): A_{Y-V} \in H_{m}(Y-V), \iota_{Y-V *}^{Y}\left(A_{Y-V}\right)=0\right\}
\end{aligned}
$$

for all $m \in \mathbb{Z}$ and for any coefficient ring.

Proof. Denote the codimension of $V$ in $X$ and $Y$ by $\mathfrak{c}, S_{X} V \approx S_{Y} V$ by $S V$, and the bundle projection map $S V \longrightarrow V$ by $q_{V}$. Mayer-Vietoris for $X \#_{\varphi} Y=(X-V) \cup_{S V}(Y-V)$ and $X \cup_{V} Y$ give a commutative pair of long exact sequences

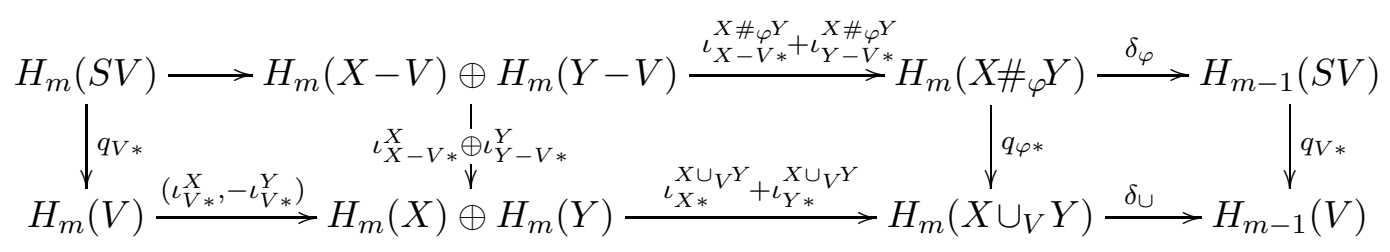

The commutativity of the middle square above implies that the second and third expressions in (4.1) are contained in the first.

Suppose $A_{\#} \in H_{m}\left(X \#_{V} Y\right)$ and $q_{\varphi *}\left(A_{\#}\right)=0$. By the proof of Mayer-Vietoris for $X \#{ }_{\varphi} Y$, there exist bordered pseudocycles

$$
f_{X}:\left(Z_{X}, \partial Z_{X}\right) \longrightarrow(X-V, S V) \quad \text { and } \quad f_{Y}:\left(Z_{Y}, \partial Z_{Y}\right) \longrightarrow(Y-V, S V)
$$

such that $\partial Z_{X}=-\partial Z_{Y}$ and

$$
f_{X} \underset{\partial Z_{X}=-\partial Z_{Y}}{\cup} f_{Y}: Z_{X} \underset{\partial Z_{X}=-\partial Z_{Y}}{\cup} Z_{Y} \longrightarrow X \#_{\varphi} Y
$$

represents the homology class $A_{\#}$. Since

$$
q_{V *}\left[\left.f_{X}\right|_{\partial Z_{X}}\right]=q_{V *} \delta_{\varphi}\left(A_{\#}\right)=\delta_{\cup} q_{\varphi *}\left(A_{\#}\right)=0,
$$

by (3.4) we can choose $f_{X}$ and $f_{Y}$ so that $f_{X}\left(\partial Z_{X}\right)=f_{Y}\left(\partial Z_{Y}\right)$ equals $\left.S V\right|_{B_{V}}$ for some class $B_{V} \in H_{m-\mathfrak{c}}(V)$. The smooth maps

$$
\iota_{X-V}^{X} \circ f_{X}: Z_{X} \longrightarrow X \quad \text { and } \quad \iota_{Y-V}^{Y} \circ f_{Y}: Z_{Y} \longrightarrow Y
$$

then determine homology classes $A_{X}$ on $X$ and $A_{Y}$ on $Y$; in the exceptional $\mathfrak{c}=1$ case, the two boundary components of these maps come with opposite signs and thus cancel. By the commutativity of the diagram on the chain level inducing the above diagram in homology,

$$
\iota_{X *}^{X \cup_{V} Y}\left(A_{X}\right)+\iota_{Y *}^{X \cup_{V} Y}\left(A_{Y}\right)=q_{\varphi *}\left(A_{\#}\right)=0 .
$$


Thus, there exists $A_{V} \in H_{m}(V)$ such that

$$
A_{X}=\iota_{V *}^{X}\left(A_{V}\right), \quad A_{Y}=-\iota_{V *}^{Y}\left(A_{V}\right) .
$$

The Mayer-Vietoris sequence (3.5) with $U=\emptyset$ then gives

$$
\left[\left.f_{X}\right|_{\partial Z_{X}}\right]=\delta_{X}\left(A_{X}\right)=0 \quad \Longrightarrow \quad \delta_{\varphi}\left(A_{\#}\right)=\left[\left.f_{X}\right|_{\partial Z_{X}}\right]=0 .
$$

The first Mayer-Vietoris sequence now implies that

$$
A_{\#}=\iota_{X-V *}^{X \#_{\varphi} Y}\left(A_{X-V}\right)+\iota_{Y-V *}^{X \#_{\varphi} Y}\left(A_{Y-V}\right)
$$

for some $A_{X-V} \in H_{m}(X-V)$ and $A_{Y-V} \in H_{m}(Y-V)$. Since

$$
\begin{aligned}
& \iota_{X *}^{X \cup_{V} Y}\left(\iota_{X-V *}^{X}\left(A_{X-V}\right)\right)+\iota_{Y *}^{X \cup_{V} Y}\left(\iota_{Y-V *}^{Y}\left(A_{Y-V}\right)\right) \\
& \quad=q_{\varphi *}\left(\iota_{X-V *}^{X \# \varphi}\left(A_{X-V}\right)\right)+q_{\varphi *}\left(\iota_{Y-V *}^{X \#_{\varphi} Y}\left(A_{Y-V}\right)\right)=q_{\varphi *}\left(A_{\#}\right)=0,
\end{aligned}
$$

the second Mayer-Vietoris sequence above implies that

$$
\iota_{X-V *}^{X}\left(A_{X-V}\right)=\iota_{V *}^{X}\left(A_{V}\right), \quad \iota_{Y-V *}^{Y}\left(A_{Y-V}\right)=-\iota_{V *}^{Y}\left(A_{V}\right)
$$

for some $A_{V} \in H_{m}(V)$. By the first equality above and the exactness of (3.5), there exists

$$
A_{S V} \in H_{m}(S V) \quad \text { s.t. } \quad \iota_{S V *}^{X-V}\left(A_{S V}\right)=A_{X-V}, \quad q_{V *}\left(A_{S V}\right)=A_{V} .
$$

By (4.2) and the first equality in (4.4),

$$
\begin{aligned}
A_{\#} & =\iota_{X-V *}^{X \#_{\varphi} Y}\left(A_{X-V}-\iota_{S V *}^{X-V}\left(A_{S V}\right)\right)+\iota_{Y-V *}^{X \#_{\varphi} Y}\left(A_{Y-V}+\iota_{S V *}^{Y-V}\left(A_{S V}\right)\right) \\
& =\iota_{Y-V *}^{X \#_{\varphi} Y}\left(A_{Y-V}+\iota_{S V *}^{Y-V}\left(A_{S V}\right)\right) .
\end{aligned}
$$

By the second equalities in (4.3) and (4.4),

$$
\iota_{Y-V *}^{Y}\left(A_{Y-V}+\iota_{S V *}^{Y-V}\left(A_{S V}\right)\right)=-\iota_{V *}^{Y}\left(A_{V}\right)+\iota_{V *}^{Y}\left(q_{V *}\left(A_{S V}\right)\right)=0 .
$$

Thus, the first expression in (4.1) is contained in the third expression and by symmetry in the second.

Taking $m=\mathfrak{c}$ in the statement of Lemma 4.1, we find that

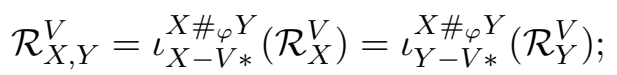

see (2.1) and (2.2) for the notation.

\subsection{The vanishing cycles}

We now focus on the $m=\mathfrak{c}$ case of Lemma 4.1, it relates $\mathcal{R}_{X, Y}^{V}$ to $\mathcal{R}_{X}^{V}$ and $\mathcal{R}_{Y}^{V}$. Let

$$
H_{\mathfrak{c}}(S V ; \mathbb{Z})_{X, Y}=\left\{A_{S V} \in H_{\mathfrak{c}}(S V ; \mathbb{Z}): q_{V *}\left(A_{S V}\right) \in \operatorname{ker} \iota_{V *}^{X} \cap \operatorname{ker} \iota_{V *}^{Y}\right\} .
$$

By the exactness of (3.5), the homomorphisms

$$
\begin{aligned}
& \iota_{S V *}^{X-V}: H_{\mathfrak{c}}(S V ; \mathbb{Z})_{X, Y} \longrightarrow \mathcal{R}_{X}^{V} \subset H_{\mathfrak{c}}(X-V ; \mathbb{Z}) \quad \text { and } \\
& \iota_{S V *}^{Y-V}: H_{\mathfrak{c}}(S V ; \mathbb{Z})_{X, Y} \longrightarrow \mathcal{R}_{Y}^{V} \subset H_{\mathfrak{c}}(Y-V ; \mathbb{Z})
\end{aligned}
$$

are surjective. 
Corollary 4.2. Let $X, Y, V$ and $\varphi$ be as in Lemma 4.1 and $\mathfrak{c}$ be the codimension of $V$ in $X$ and $Y$.

(1) The subgroup $\mathcal{R}_{X, Y}^{V} \subset H_{\mathfrak{c}}\left(X \#{ }_{\varphi} Y ; \mathbb{Z}\right)$ is isomorphic to the cokernel of the homomorphism

$$
H_{\mathfrak{c}}(S V ; \mathbb{Z})_{X, Y} \longrightarrow \mathcal{R}_{X}^{V} \oplus \mathcal{R}_{Y}^{V}, \quad A_{S V} \longrightarrow\left(\iota_{S V *}^{X-V}\left(A_{S V}\right),-\iota_{S V *}^{Y-V}\left(A_{S V}\right)\right) .
$$

(2) If either $\mathcal{R}_{X}^{V}=\{0\}$ or $\mathcal{R}_{Y}^{V}=\{0\}$, then $\mathcal{R}_{X, Y}^{V}=\{0\}$ and the homomorphism

$$
\#: H_{\mathfrak{c}}(X ; \mathbb{Z}) \times_{V} H_{\mathfrak{c}}(Y ; \mathbb{Z}) \longrightarrow H_{\mathfrak{c}}\left(X \#{ }_{\varphi} Y ; \mathbb{Z}\right), \quad\left(A_{X}, A_{Y}\right) \longrightarrow A_{X} \#_{\varphi} A_{Y}
$$

induced by gluing representatives of homology classes along $V$, is well-defined.

Proof. (1) By the $m=\mathfrak{c}$ case of Lemma 4.1, this claim is equivalent to

$$
\begin{aligned}
& \left.\left\{\left(A_{X-V}, A_{Y-V}\right) \in \mathcal{R}_{X}^{V} \oplus \mathcal{R}_{Y}^{V}: \stackrel{\iota_{X-V *}^{X \#_{\varphi} Y}}{\iota_{X-V}}\right)+A_{X-V *}^{X \#_{\varphi} Y}\left(A_{Y-V}\right)=0\right\} \\
& =\left\{\left(\iota_{S V *}^{X-V}\left(A_{S V}\right),-\iota_{S V *}^{Y-V}\left(A_{S V}\right)\right): A_{S V} \in H_{\mathfrak{c}}(S V ; \mathbb{Z}), q_{V *}\left(A_{S V}\right) \in \operatorname{ker} \iota_{V *}^{X} \cap \operatorname{ker} \iota_{V *}^{Y}\right\} .
\end{aligned}
$$

Let $A_{X-V} \in H_{\mathfrak{c}}(X-V ; \mathbb{Z})$ and $A_{Y-V} \in H_{\mathfrak{c}}(Y-V ; \mathbb{Z})$. By the Mayer-Vietoris sequence for $X \#{ }_{\varphi} Y$ in the proof of Lemma 4.1 .

$$
\begin{aligned}
& \iota_{X-V *}^{X \# \varphi}\left(A_{X-V}\right)+\iota_{Y-V *}^{X \# \varphi Y}\left(A_{Y-V}\right)=0 \quad \Longleftrightarrow \\
& \left(A_{X-V}, A_{Y-V}\right)=\left(\iota_{S V *}^{X-V}\left(A_{S V}\right),-\iota_{S V *}^{Y-V}\left(A_{S V}\right)\right) \quad \text { for some } A_{S V} \in H_{\mathfrak{c}}(S V ; \mathbb{Z}) \text {. }
\end{aligned}
$$

For any $A_{S V} \in H_{\mathfrak{c}}(S V ; \mathbb{Z})$, the commutativity of the first square in the diagram of short exact sequences in the proof of Lemma 4.1 implies that

$$
\left(\iota_{S V *}^{X-V}\left(A_{S V}\right),-\iota_{S V *}^{Y-V}\left(A_{S V}\right)\right) \in \mathcal{R}_{X}^{V} \oplus \mathcal{R}_{Y}^{V} \quad \Longleftrightarrow \quad q_{V *}\left(A_{S V}\right) \in \operatorname{ker} \iota_{V *}^{X} \cap \operatorname{ker} \iota_{V *}^{Y} .
$$

The last two statements give (4.10).

(2) The second claim of this corollary follows from the first and the surjectivity of the homomorphisms (4.7).

Remark 4.3. The patching map $\varphi$ covering the identity on $V$ does not effect the homomorphism (4.8), as the former corresponds to a trivialization of an $S^{c-1}$-bundle over $S^{1}$ for each fixed element of $\mathcal{R}_{X}^{V} \oplus \mathcal{R}_{Y}^{V}$. Thus, $\mathcal{R}_{X, Y}^{V}$ does not depend on the choice of $\varphi$. However, it may depend on the identification of the copies of $V$ in $X$ and $Y$, as illustrated in Example 4.7 .

We next restrict to the setting of Corollary 3.2 with $U=\emptyset$; the last restriction is not necessary, but the case $U=\emptyset$ suffices for our purposes. Thus, suppose that $X, Y$, and $V$ are compact and oriented. Define

$$
\Delta_{X, Y}^{V}: H_{1}(V ; \mathbb{Z})_{X} \oplus H_{1}(V ; \mathbb{Z})_{Y} \longrightarrow \frac{H_{1}(V ; \mathbb{Z})}{H_{X}^{V}+H_{Y}^{V}}, \quad\left(\left[\gamma_{X}\right]_{H_{X}^{V}},\left[\gamma_{Y}\right]_{H_{Y}^{V}}\right) \longrightarrow\left[\gamma_{X}-\gamma_{Y}\right]_{H_{X}^{V}+H_{Y}^{V}}
$$

Denote by $\bar{H}_{X, Y}^{V}$ the image of the composition

$$
H_{\mathfrak{c}}(S V ; \mathbb{Z})_{X, Y} \stackrel{\left(\iota_{S V^{*}}^{X-V},-\iota_{S V^{*}}^{Y-V}\right)}{\longrightarrow} \mathcal{R}_{X}^{V} \oplus \mathcal{R}_{Y}^{V} \stackrel{\approx}{\longrightarrow} H_{1}(V ; \mathbb{Z})_{X} \oplus H_{1}(V ; \mathbb{Z})_{Y} \stackrel{\Delta_{X, Y}^{V}}{\longrightarrow} \frac{H_{1}(V ; \mathbb{Z})}{H_{X}^{V}+H_{Y}^{V}},
$$


with the second arrow above given by the isomorphisms of Corollary 3.2 . Let $H_{X, Y}^{V} \subset H_{1}(V ; \mathbb{Z})$ be the preimage of $\bar{H}_{X, Y}^{V}$ under the quotient projection

$$
H_{1}(V ; \mathbb{Z}) \longrightarrow \frac{H_{1}(V ; \mathbb{Z})}{H_{X}^{V}+H_{Y}^{V}}
$$

In particular,

$$
H_{X}^{V}+H_{Y}^{V} \subset H_{X, Y}^{V} \subset H_{1}(V ; \mathbb{Z})
$$

and the first inclusion is an equality if either $\iota_{V *}^{X}$ or $\iota_{V *}^{Y}$ is injective on $H_{\mathfrak{c}}$; see the proof of Corollary 4.4 below.

Corollary 4.4. Let $X$ and $Y$ be compact oriented manifolds, $V \subset X, Y$ be a compact oriented submanifold, and $\varphi: S_{X} V \longrightarrow S_{Y} V$ be an orientation-reversing diffeomorphism commuting with the projections to $V$.

(1) The isomorphisms of Corollary 3.2 for $(X, V)$ and $(Y, V)$ induce a commutative diagram

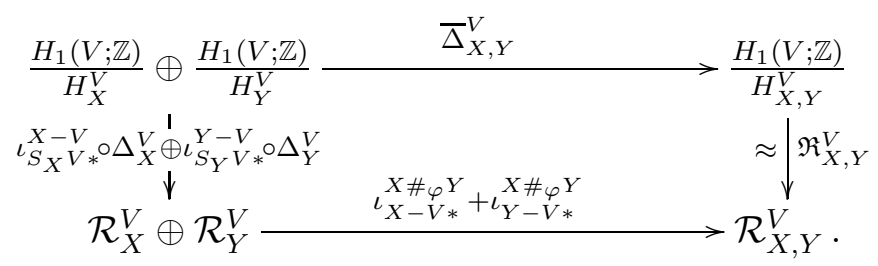

(2) If either of $\iota_{V *}^{X}$ or $\iota_{V *}^{Y}$ is injective on $H_{\mathfrak{c}}$, then $\mathcal{R}_{X, Y}^{V}$ is isomorphic to the cokernel of the homomorphism

$$
H_{1}(V ; \mathbb{Z}) \longrightarrow H_{1}(V ; \mathbb{Z})_{X} \oplus H_{1}(V ; \mathbb{Z})_{Y}, \quad \gamma \longrightarrow\left([\gamma]_{X},[\gamma]_{Y}\right),
$$

with $[\gamma]_{X}$ and $[\gamma]_{Y}$ denoting the corresponding cosets of $\gamma$.

Proof. (1) The image of ker $\Delta_{X, Y}^{V}$ under the isomorphisms of Corollary 3.2 is given by

$$
\begin{aligned}
\left\{\left(\iota_{S_{X} V *}^{X-V}\left(\Delta_{X}^{V}(\gamma)\right), \iota_{S_{Y} V *}^{Y-V}\left(\Delta_{Y}^{V}(\gamma)\right)\right): \gamma \in H_{1}(V ; \mathbb{Z})\right\} \\
=\left\{\left(\iota_{S_{X} V *}^{X-V}\left(\Delta_{X}^{V}(\gamma)\right),-\iota_{S_{Y} V *}^{Y-V}\left(\Delta_{X}^{V}(\gamma)\right)\right): \gamma \in H_{1}(V ; \mathbb{Z})\right\},
\end{aligned}
$$

since $\varphi$ is orientation-reversing. Thus, this image is contained in the image of the homomorphism (4.8). The first claim now follows from Corollary 4.2) (1) and the definition of $H_{X, Y}^{V}$ above.

(2) If either $\iota_{V *}^{X}$ or $\iota_{V *}^{Y}$ is injective on $H_{\mathfrak{c}}$,

$$
H_{\mathfrak{c}}(S V ; \mathbb{Z})_{X, Y}=\operatorname{ker} q_{V *}=\left\{\Delta_{X}^{V}(\gamma): \gamma \in H_{1}(V ; \mathbb{Z})\right\}
$$

the last equality holds by the exactness of (3.4). Thus, the image of the homomorphism (4.8) is given by (4.13) and $H_{X, Y}^{V}=H_{X}^{V}+H_{Y}^{V}$. The second claim of this corollary now follows from the first.

Corollary 4.5. If $X$ is a compact oriented manifold, $V \subset X$ is a compact oriented submanifold, and $\varphi: S_{X} V \longrightarrow S_{X} V$ is an orientation-reversing diffeomorphism commuting with the projection to $V$, then $\mathcal{R}_{X, X}^{V} \approx H_{1}(V ; \mathbb{Z})_{X}$. 
Proof. Since $\iota_{V *}^{X} \circ q_{V *}=\iota_{X-V *}^{X} \circ \iota_{S V *}^{X-V}$,

$$
H_{\mathfrak{c}}(S V ; \mathbb{Z})_{X, X}=\left\{A_{S V} \in H_{\mathfrak{c}}(S V ; \mathbb{Z}): \iota_{S V *}^{X-V}\left(A_{S V}\right) \in \mathcal{R}_{X}^{V}\right\} .
$$

Thus, $\bar{H}_{X, Y}^{V}$ is the image of the diagonal subgroup under the homomorphism

$$
H_{1}(V ; Z)_{X} \oplus H_{1}(V ; Z)_{X} \longrightarrow H_{1}(V ; Z)_{X}, \quad\left(\left[\gamma_{1}\right],\left[\gamma_{2}\right]\right)=\left[\gamma_{1}-\gamma_{2}\right] .
$$

Therefore, $H_{X, X}^{V}=H_{X}^{V}$ and the claim follows from Corollary 4.4](1).

Example 4.6. Let $\widehat{\mathbb{P}}_{9}^{2}$ be a rational elliptic surface and $F \subset \widehat{\mathbb{P}}_{9}^{2}$ be a smooth fiber as in Example 3.5. For the standard identification of $F$ in two copies of $\widehat{\mathbb{P}}_{9}^{2}$, the homomorphism (4.12) can be written as

$$
\mathbb{Z}^{2} \longrightarrow \mathbb{Z}^{2} \oplus \mathbb{Z}^{2}, \quad(a, b) \longrightarrow((a, b),(a, b))
$$

Thus, $\mathcal{R}_{\widehat{\mathbb{P}}_{9}^{2}, \widehat{\mathbb{P}}_{9}^{2}}^{F} \approx \mathbb{Z}^{2}$; this also follows from Corollary 4.5.

Example 4.7. Let $F, X$, and $F_{0}, F_{\infty} \subset X$ be as in Example 3.6 and $Y=X$. For the standard identification of the copies of $F_{0} \cup F_{\infty}$ in $X$ and in $Y$, the homomorphism (4.12) can be written as

$$
H_{1}(F ; \mathbb{Z}) \oplus H_{1}(F ; \mathbb{Z}) \longrightarrow H_{1}(F ; \mathbb{Z}) \oplus H_{1}(F ; \mathbb{Z}), \quad\left(\gamma_{0}, \gamma_{\infty}\right) \longrightarrow\left(\gamma_{0}-\gamma_{\infty}, \gamma_{0}-\gamma_{\infty}\right) .
$$

Thus, $\mathcal{R}_{X, X}^{F_{0} \cup F_{\infty}} \approx H_{1}(F ; \mathbb{Z})$ in this case, with the isomorphism induced by the homomorphism

$$
H_{1}(F ; \mathbb{Z}) \oplus H_{1}(F ; \mathbb{Z}) \longrightarrow H_{1}(F ; \mathbb{Z}), \quad(\alpha, \beta) \longrightarrow \alpha-\beta .
$$

For an arbitrary identification of the two copies of $F_{0} \cup F_{\infty}$, the above homomorphism becomes

$$
H_{1}(F ; \mathbb{Z}) \oplus H_{1}(F ; \mathbb{Z}) \longrightarrow H_{1}(F ; \mathbb{Z}) \oplus H_{1}(F ; \mathbb{Z}), \quad\left(\gamma_{0}, \gamma_{\infty}\right) \longrightarrow\left(\gamma_{0}-\gamma_{\infty}, \gamma_{0}-\phi_{*} \gamma_{\infty}\right),
$$

for some diffeomorphism $\phi: F \longrightarrow F$. For example, if $F=\mathbb{T}^{2}$ is the two-torus, $\mathcal{R}_{X, X}^{V} \approx \mathbb{Z}^{2}$ for the standard identification, but $\mathcal{R}_{X, X}^{F_{0} \cup F_{\infty}}$ can be $\mathbb{Z}$ or $\{0\}$ for other identifications.

Example 4.8. Suppose $X$ is an oriented manifold and $Z \subset X$ is a compact submanifold so that the normal bundle $\mathcal{N}_{X} Z$ admits a complex structure. Fix a complex structure in $\mathcal{N}_{X} Z$ and an identification of the unit disk bundle $D\left(\mathcal{N}_{X} Z\right)$ of $\mathcal{N}_{X} Z$ with a neighborhood of $Z$ in $X$. Let

$$
\mathbb{P}_{X} Z=\mathbb{P}\left(\mathcal{N}_{X} Z \times \mathbb{C}\right)=\mathbb{P}\left(\mathcal{N}_{X} Z \oplus Z \times \mathbb{C}\right), \quad V=\mathbb{P}\left(\mathcal{N}_{X} Z\right) \subset \mathbb{P}\left(\mathcal{N}_{X} Z \times \mathbb{C}\right),
$$

and $\mathrm{Bl}_{Z} X$ be the manifold obtained from $X$ by replacing $D\left(\mathcal{N}_{X} Z\right) \subset X$ with the disk bundle of the complex tautological line bundle $\gamma \longrightarrow V$ (which has the same boundary consisting of the unit vectors in $\left.\mathcal{N}_{X} V\right)$. Thus,

$$
\mathcal{N}_{\mathrm{Bl}_{Z} X} V=\gamma, \quad \mathcal{N}_{\mathbb{P}_{X} Z} V=\gamma^{*}, \quad \text { and } \quad X=\mathrm{Bl}_{Z} X \#_{\varphi} \mathbb{P}_{X} Z,
$$

for an orientation-reversing diffeomorphism $\varphi: S_{\mathrm{Bl}_{Z} X} V \longrightarrow S_{\mathbb{P}_{X} Z} V$ induced by the canonical isomorphism $\gamma \otimes \gamma^{*}=V \times \mathbb{C}\left(\right.$ e.g. $\{\varphi(v)\} v=1$ for all $\left.v \in S_{\mathrm{Bl}_{Z} X} V\right)$. By Corollary 4.2] (2)] and Example 3.4,

$$
\mathcal{R}_{\mathrm{Bl}_{Z} X, \mathbb{P}_{X} Z}^{V}=\{0\},
$$

i.e. there are no rim tori in this case. A geometric reasoning for this conclusion is given in the proof of [11, Lemma 2.11]. If $(X, \omega)$ is a symplectic manifold and $Z \subset X$ is a symplectic submanifold, the construction of [13, Section 7.1] endows $\mathrm{Bl}_{Z} X$ with a symplectic form $\omega_{Z, \epsilon} ;\left(\mathrm{Bl}_{Z} X, \omega_{Z, \epsilon}\right)$ is then called a symplectic blowup of $(X, \omega)$ along $Z$. 


\subsection{Changes in rim tori}

We continue with the setup of Lemma 4.1. If $U \subset X$ and $W \subset Y$ are closed submanifolds of codimension $\mathfrak{c}$ disjoint from $V$, then $U, W \subset X \# \#_{\varphi} Y$ are also disjoint submanifolds of codimension $\mathfrak{c}$. Below we relate the rim tori for $\left(X \#_{\varphi} Y, U \cup W\right)$ to rim tori in $X$ and $Y$ and to the vanishing cycles in $X \#_{\varphi} Y$. This relation is described by the squares of exact sequences in Figures 3 and 4 . Such a relation is needed to make sense of the rim tori refinement to the symplectic sum formula for relative GW-invariants suggested by [8, (12.7)] and of the convolution product on rim tori covers appearing above [8, (11.5)]; see [4, Section 5.2] for details.

With notation as in (4.6), let

$$
\dot{H}_{X, Y}^{S V}=\left\{\left(\iota_{S V *}^{X-V}\left(A_{S V}\right),-\iota_{S V *}^{Y-V}\left(A_{S V}\right)\right): A_{S V} \in H_{\mathfrak{c}}(S V ; \mathbb{Z})_{X, Y}\right\} \subset \mathcal{R}_{X}^{V} \oplus \mathcal{R}_{Y}^{V}
$$

With $U$ and $W$ as in the previous paragraph fixed, define

$$
\begin{aligned}
\widetilde{H}_{X, Y}^{S V} & =\left\{\left(\iota_{S V *}^{X-U \cup V}\left(A_{S V}\right),-\iota_{S V *}^{Y-V \cup W}\left(A_{S V}\right)\right): A_{S V} \in H_{\mathfrak{c}}(S V ; \mathbb{Z})_{X, Y}\right\}, \\
\stackrel{\circ}{H}_{X, Y}^{S V} & =\widetilde{H}_{X, Y}^{S V} \cap \mathcal{R}_{X-V}^{U} \oplus \mathcal{R}_{Y-V}^{W} \subset \mathcal{R}_{X}^{U \cup V} \oplus \mathcal{R}_{Y}^{V \cup W}, \\
\widetilde{\mathcal{R}}_{X \#{ }_{\varphi} Y}^{U \cup W} & =\left\{\iota_{X-U \cup V *}^{X \# \varphi Y-U \cup W}\left(A_{X}\right)+\iota_{Y-V \cup W}^{X \#{ }_{\varphi} Y-U \cup W}\left(A_{Y}\right):\left(A_{X}, A_{Y}\right) \in \mathcal{R}_{X}^{U \cup V} \oplus \mathcal{R}_{Y}^{V \cup W}\right\} .
\end{aligned}
$$

By Proposition 4.9 below and (2.2),

$$
\widetilde{\mathcal{R}}_{X \# \varphi}^{U \cup W}=\operatorname{ker}\left\{q_{\varphi *} \circ \iota_{X \#_{\varphi} Y-U \cup W *}^{X \#_{\varphi} Y}: H_{\mathfrak{c}}\left(X \#_{\varphi} Y-U \cup W ; \mathbb{Z}\right) \longrightarrow H_{\mathfrak{c}}\left(X \cup_{V} Y ; \mathbb{Z}\right)\right\} .
$$

Proposition 4.9. Suppose $X$ and $Y$ are manifolds, $U, V \subset X$ and $V, W \subset Y$ are closed disjoint submanifolds of the same codimension $\mathfrak{c}$, and $\varphi: S_{X} V \longrightarrow S_{Y} V$ is a diffeomorphism commuting with the projections to $V$. Then, the homology homomorphisms induced by inclusions give rise to the commutative square of short exact sequences of Figure 3.

Proof. The commutativity of all four squares is immediate. The left column is exact by (4.15) and (2.1), while the middle column is exact because the right column in Figure 2 is (which does not require the additional assumptions of Corollary 3.31). The bottom row is exact by Corollary 4.2](1), The middle row is exact at $\widetilde{H}_{X, Y}^{S V}$ and $\widetilde{\mathcal{R}}_{X \# \varphi Y}^{U \cup W}$ by the definitions of the two modules. It is exact at $\mathcal{R}_{X}^{U \cup V} \oplus \mathcal{R}_{Y}^{V \cup W}$ by the exactness of the Mayer-Vietoris sequence for $X \#{ }_{\varphi} Y$ in the proof of Lemma 4.1 with $(X, Y)$ replaced by $(X-U, Y-W)$; see the proof of Corollary 4.2 ,

The top row is exact at $\stackrel{\circ}{H} \underset{X, Y}{S V}$ by the definition of this module. It is exact at $\mathcal{R}_{X-V}^{U} \oplus \mathcal{R}_{Y-V}^{W}$ by the same reasoning as in the proof of Corollary 4.2. In order to establish the exactness of the top row at the last position, we use the commutative diagram

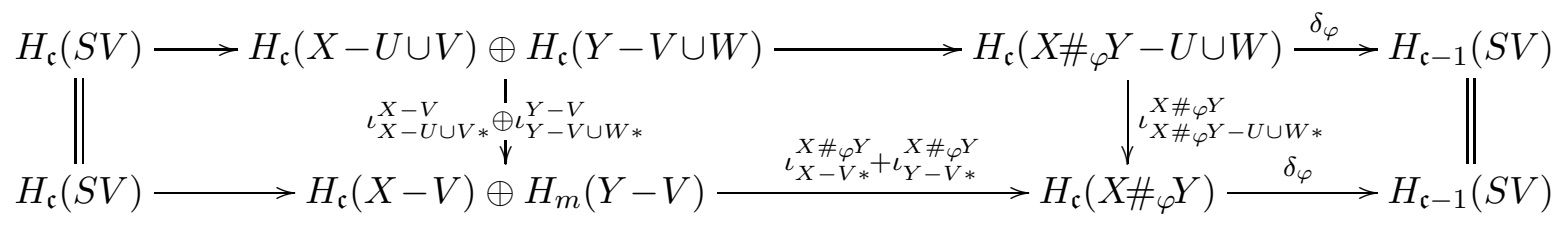

of the Mayer-Vietoris sequences for $X \#_{\varphi} Y$ and $(X-U) \#_{\varphi}(Y-W)$. 


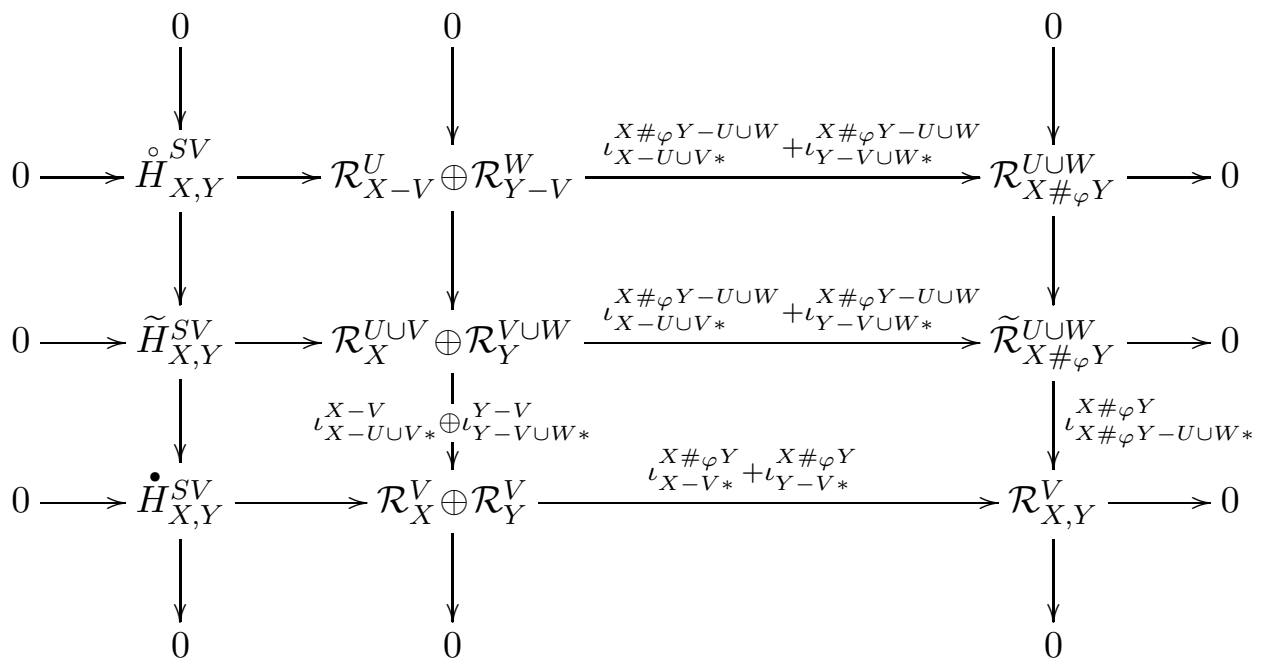

Figure 3: Rim tori under gluing: general case.

If $A \in \mathcal{R}_{X \#{ }_{\varphi} Y}^{U \cup W}$, then

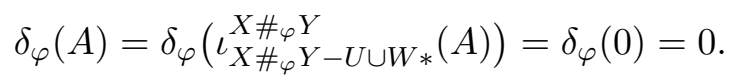

By the exactness of the first sequence above, this implies that

$$
A=\iota_{X-U \cup V *}^{X \#_{\varphi} Y-U \cup W}\left(A_{X}\right)+\iota_{Y-V \cup W *}^{X \#_{\varphi} Y-U \cup W}\left(A_{Y}\right)
$$

for some

$$
A_{X} \in H_{\mathfrak{c}}(X-U \cup V ; \mathbb{Z}), \quad A_{Y} \in H_{\mathfrak{c}}(Y-V \cup W ; \mathbb{Z}) .
$$

By the commutativity of the middle square in the Mayer-Vietoris diagram,

$$
\begin{aligned}
\left.\stackrel{\iota_{X-V *}^{X \#_{\varphi} Y}\left(\iota_{X-V}^{X-V}\right.}{\iota_{X-U V *}}\left(A_{X}\right)\right)+\iota_{Y-V *}^{V \#_{\varphi} Y}\left(\iota_{Y-V \cup W *}^{Y-V}\left(A_{Y}\right)\right) \\
=\iota_{X \#_{\varphi} Y-U \cup W *}^{X \#_{X} Y}\left(\iota_{X-U \cup V *}^{X \#_{\varphi} Y-U \cup W}\left(A_{X}\right)\right)+\iota_{X \#_{\varphi} Y-U \cup W *}^{X \#_{\varphi} Y}\left(\iota_{Y-V \cup W *}^{X \#_{\varphi} Y-U \cup W}\left(A_{Y}\right)\right) \\
=\iota_{X \#_{\varphi} Y-U \cup W *}^{X \#_{\varphi} Y}(A)=0 .
\end{aligned}
$$

Thus, the exactness of the bottom row implies that

$$
\iota_{X-U \cup V *}^{X-V}\left(A_{X}\right)=\iota_{S V *}^{X-V}\left(A_{S V}\right), \quad \iota_{Y-V \cup W *}^{Y-V}\left(A_{Y}\right)=-\iota_{S V *}^{Y-V}\left(A_{S V}\right)
$$

for some $A_{S V} \in H_{\mathfrak{c}}(S V ; \mathbb{Z})$. By the commutativity of the left square and (4.17),

$$
A_{X}=A_{X}^{\prime}+\iota_{S V *}^{X-U \cup V}\left(A_{S V}\right), \quad A_{Y}=A_{Y}^{\prime}-\iota_{S V *}^{Y-V \cup W}\left(A_{S V}\right)
$$

for some $A_{X}^{\prime} \in \mathcal{R}_{X-V}^{U}$ and $A_{Y}^{\prime} \in \mathcal{R}_{Y-V}^{W}$. By (4.16) and (4.18),

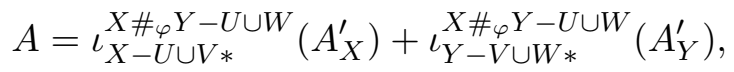

and so the top row is exact at the last position. 


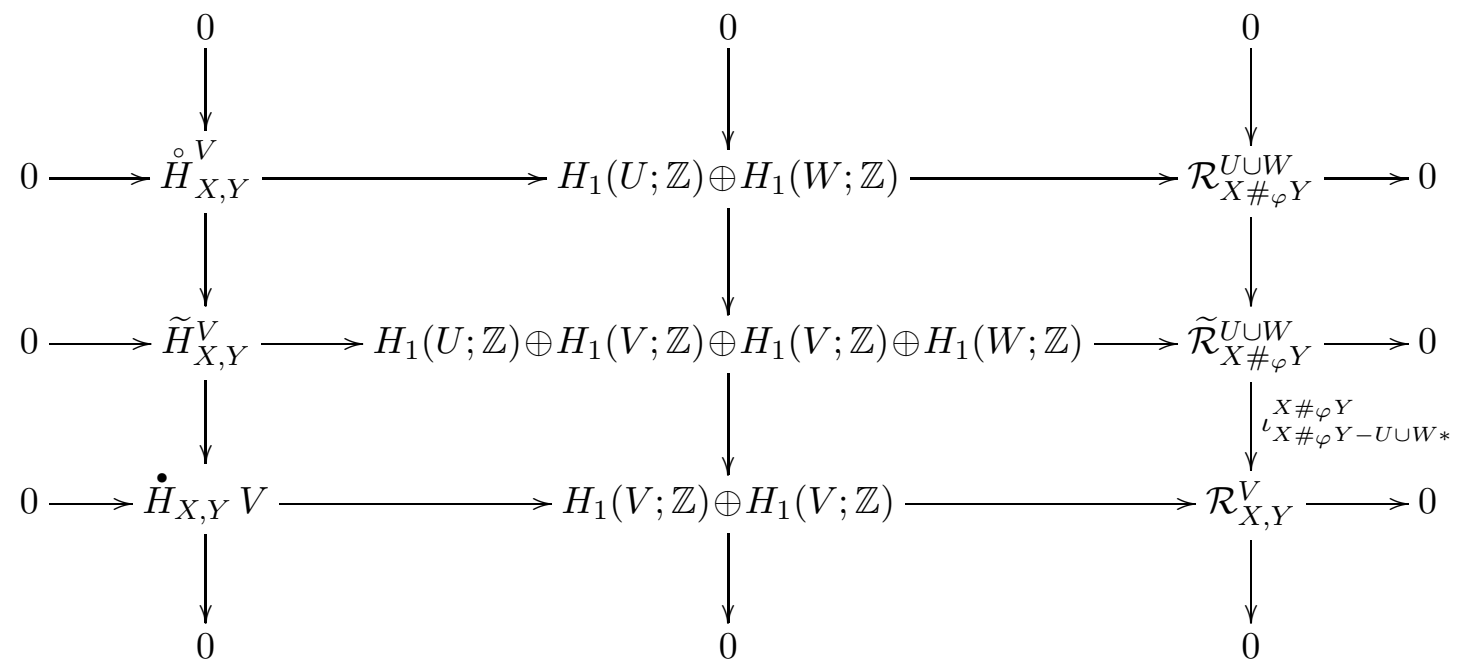

Figure 4: Rim tori under gluing: compact oriented case.

Finally, the first homomorphism in the right column is the inclusion of a submodule. This column is exact at the middle and last positions by the commutativity of the diagram and the exactness of the remaining sequences.

We now restrict to the settings of Corollaries 3.2 and 4.4. Thus, suppose that $X, Y, U, V$, and $W$ are compact and oriented. Let

$$
\begin{gathered}
\stackrel{\circ}{H}_{X, Y}^{V} \subset H_{1}(U ; \mathbb{Z}) \oplus H_{1}(W ; \mathbb{Z}), \quad \dot{H}_{X, Y}^{V} \subset H_{1}(V ; \mathbb{Z}) \oplus H_{1}(V ; \mathbb{Z}), \\
\text { and } \quad \widetilde{H}_{X, Y}^{V} \subset H_{1}(U ; \mathbb{Z}) \oplus H_{1}(V ; \mathbb{Z}) \oplus H_{1}(V ; \mathbb{Z}) \oplus H_{1}(W ; \mathbb{Z})
\end{gathered}
$$

be the preimages of

$$
\stackrel{\circ}{H}_{X, Y}^{S V} \subset \mathcal{R}_{X-V}^{U} \oplus \mathcal{R}_{Y-V}^{W}, \quad H_{X, Y}^{S V} \subset \mathcal{R}_{X}^{V} \oplus \mathcal{R}_{Y}^{V}, \quad \text { and } \quad \widetilde{H}_{X, Y}^{V} \subset \mathcal{R}_{X}^{U \cup V} \oplus \mathcal{R}_{Y}^{V \cup W},
$$

respectively, under the homomorphisms as in Corollary 3.2. The commutative square of short exact sequences of Figure 3 then induces the commutative square of short exact sequences of Figure 4 .

Example 4.10. Let $X=\widehat{\mathbb{P}}_{9}^{2}$ be the rational elliptic surface of Examples 3.5 and 4.6 with smooth fiber $F \subset \widehat{\mathbb{P}}_{9}^{2}, Y=\mathbb{P}^{1} \times \mathbb{T}^{2}$, and $F_{0}, F_{\infty} \subset \mathbb{P}^{1} \times \mathbb{T}^{2}$ be as in Examples 3.6 and 4.7. We take $U=\emptyset$, $W=F_{\infty}, V=F \subset X$, and $V=F_{0} \subset Y$. In this case, $\mathfrak{c}=2$, the homomorphisms

$$
\iota_{V *}^{X}: H_{2}(V ; \mathbb{Z}) \longrightarrow H_{2}(X ; \mathbb{Z}) \quad \text { and } \quad \iota_{V *}^{Y}: H_{2}(V ; \mathbb{Z}) \longrightarrow H_{2}(Y ; \mathbb{Z})
$$

are injective, and the homomorphisms

$$
\begin{gathered}
\Delta_{X}^{V}: H_{1}(V ; \mathbb{Z}) \longrightarrow H_{2}(S V ; \mathbb{Z})_{X, Y}, \quad \iota_{S V *}^{Y-V \cup W}: H_{2}(S V ; \mathbb{Z})_{X, Y} \longrightarrow H_{2}(Y-V \cup W ; \mathbb{Z}), \\
\quad \text { and } \quad \iota_{S V *}^{X-U \cup V}=\iota_{S V *}^{X-V}: H_{2}(S V ; \mathbb{Z})_{X, Y} \longrightarrow H_{2}(X-U \cup V ; \mathbb{Z})=H_{2}(X-V ; \mathbb{Z}),
\end{gathered}
$$




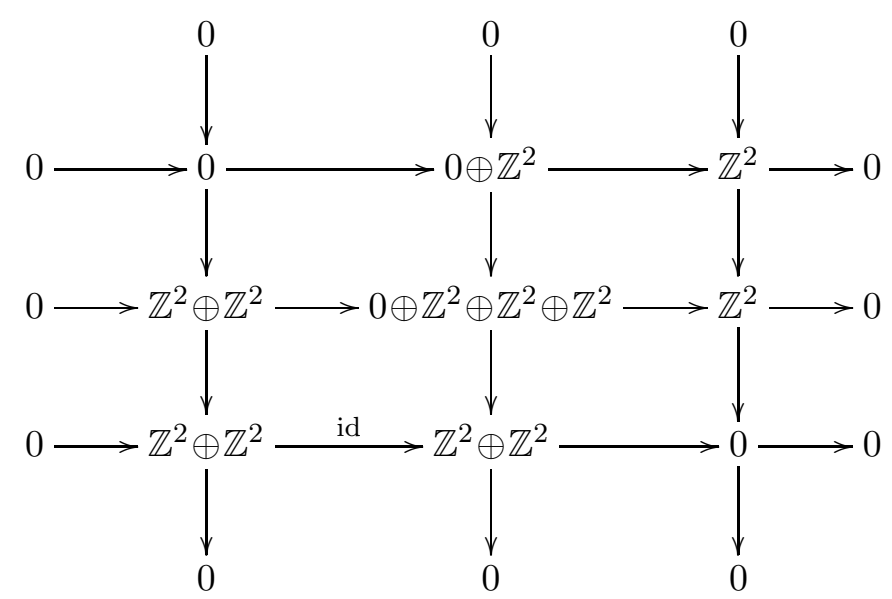

Figure 5: Rim tori under gluing for $\left(\widehat{\mathbb{P}}_{9}^{2}, F\right)=\left(\widehat{\mathbb{P}}_{9}^{2}, \emptyset\right) \# \varphi\left(\mathbb{P}^{1} \times \mathbb{T}^{2}, F_{\infty}\right)$.

are isomorphisms. The exact square in Figure 4 then specializes to the exact square of Figure 5 , The two homomorphisms in the middle column are given by

$$
(0, \gamma) \longrightarrow(0,0,0, \gamma) \quad \text { and } \quad\left(0, \gamma_{1}, \gamma_{2}, \gamma_{3}\right) \longrightarrow\left(\gamma_{1}, \gamma_{2}\right)
$$

The two homomorphisms in the middle row are given by

$$
\left(\gamma_{1}, \gamma_{2}\right) \longrightarrow\left(0, \gamma_{1}, \gamma_{1}+\gamma_{2}, \gamma_{2}\right) \quad \text { and } \quad\left(0, \gamma_{1}, \gamma_{2}, \gamma_{3}\right) \longrightarrow \gamma_{1}-\gamma_{2}+\gamma_{3}
$$

the restrictions of the first homomorphism to the last component and of the second homomorphism to the last two components correspond to (3.9) and (3.10), respectively. For the standard identification $\varphi, \widehat{\mathbb{P}}_{9}^{2}=\widehat{\mathbb{P}}_{9}^{2} \# \varphi\left(\mathbb{P}^{1} \times \mathbb{T}^{2}\right)$.

\subsection{Changes in cohomology}

By Lemma 4.1 and the exactness of (3.5),

$$
\begin{aligned}
\operatorname{ker}\left\{q_{\varphi *}: H_{m}\left(X \#{ }_{\varphi} Y ; \mathbb{Z}\right) \longrightarrow H_{m}\left(X \cup_{V} Y ; \mathbb{Z}\right)\right\} \\
\quad=\left\{\iota_{S V^{*}}^{X \#_{\varphi} Y}\left(A_{S V}\right): A_{S V} \in \operatorname{ker}\left\{q_{V}: H_{m}(S V ; \mathbb{Z}) \longrightarrow H_{m}(V ; \mathbb{Z})\right\}\right\} .
\end{aligned}
$$

Lemma 4.11 below, which describes cohomology classes used as primary inputs for GW-invariants in the symplectic sum formula, can be seen as the dual of (4.19). The analogue of this lemma with field coefficients, which would be sufficient for the purposes of the symplectic sum formula, follows immediately by dualizing (4.1) with coefficients in the same field. Similarly, the proof of Lemma 4.11 can be viewed as the dual version of the proof of Lemma 4.1, but we include it for the sake of completeness; like the proof of Lemma 4.1, it contains a delicate step.

Lemma 4.11. If $X$ and $Y$ are manifolds, $V \subset X, Y$ is a closed submanifold, $\varphi: S_{X} V \longrightarrow S_{Y} V$ is a diffeomorphism commuting with the projections to $V$, and $q_{\varphi}: X \#_{\varphi} Y \longrightarrow X \cup_{V} Y$ is a collapsing map, then

$$
\left\{q_{\varphi}^{*} \alpha_{\cup}: \alpha_{\cup} \in H^{*}\left(X \cup_{V} Y ; \mathbb{Z}\right)\right\}=\left\{\alpha_{\#} \in H^{*}\left(X \#{ }_{\varphi} Y ; \mathbb{Z}\right):\left.\alpha_{\#}\right|_{S V} \in q_{V}^{*}\left(H^{*}(V ; \mathbb{Z})\right)\right\},
$$


where $S V \subset X \#_{\varphi} Y$ is the sphere bundle $S_{X} V \approx S_{Y} V$.

Proof. The commutativity of the diagram

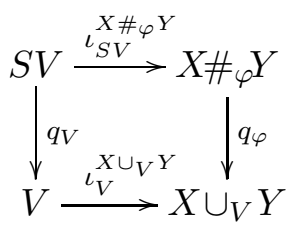

implies that the left-hand side of (4.20) is contained in the right-hand side. Below we confirm the opposite inclusion.

We will use the commutative diagram of the Mayer-Vietoris cohomology sequences for $X \cup_{V} Y$ and $X \#_{\varphi} Y=(X-V) \cup_{S V}(Y-V)$,

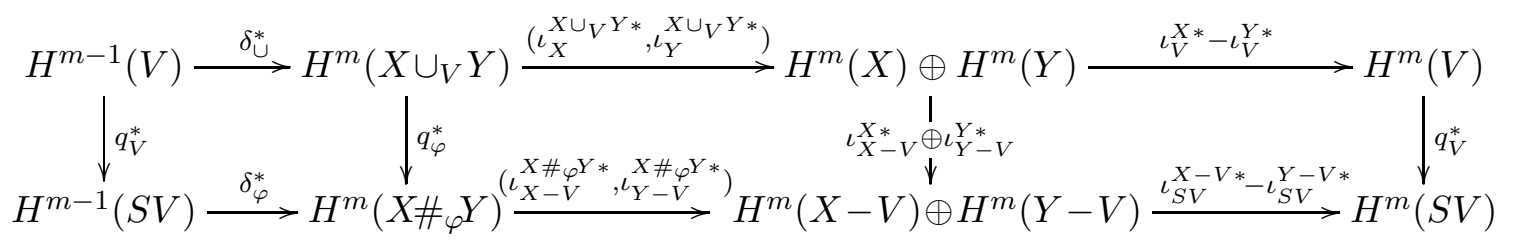

where $H^{*}$ denotes integral cohomology groups. Suppose

$$
\alpha_{\#} \in H^{*}\left(X \#{ }_{\varphi} Y ; \mathbb{Z}\right), \quad \alpha_{V} \in H^{*}(V ; \mathbb{Z}),\left.\quad \alpha_{\#}\right|_{S V}=q_{V}^{*} \alpha_{V}
$$

By Mayer-Vietoris for $M=(M-V) \cup_{S V} V$, where $M=X, Y$,

$$
H^{m}(M ; \mathbb{Z}) \stackrel{\left(\iota_{M-V}^{M *}, \iota_{V}^{M *}\right)}{\longrightarrow} H^{m}(M-V ; \mathbb{Z}) \oplus H^{m}(V ; \mathbb{Z}) \stackrel{\iota_{S V}^{M-V *}-q_{V}^{*}}{\longrightarrow} H^{m}(S V ; \mathbb{Z}),
$$

there exist $\alpha_{X} \in H^{m}(X ; \mathbb{Z})$ and $\alpha_{Y} \in H^{m}(Y ; \mathbb{Z})$ such that

$$
\left.\alpha_{X}\right|_{X-V}=\left.\alpha_{\#}\right|_{X-V},\left.\quad \alpha_{Y}\right|_{Y-V}=\left.\alpha_{\#}\right|_{Y-V},\left.\quad \alpha_{X}\right|_{V},\left.\alpha_{Y}\right|_{V}=\alpha_{V} .
$$

By the last equality in (4.21) and the Mayer-Vietoris sequence for $X \cup_{V} Y$ above, there exists

$$
\alpha_{\cup} \in H^{m}\left(X \cup_{V} Y ; \mathbb{Z}\right) \quad \text { s.t. }\left.\quad \alpha_{\cup}\right|_{X}=\alpha_{X},\left.\quad \alpha_{\cup}\right|_{Y}=\alpha_{Y} .
$$

By the commutativity of the middle square in the above diagram, the two equalities in (4.22), and the first two equalities in (4.21),

$$
\left.\left(\alpha_{\#}-q_{\varphi}^{*} \alpha_{\cup}\right)\right|_{X-V}=0 \quad \text { and }\left.\quad\left(\alpha_{\#}-q_{\varphi}^{*} \alpha_{\cup}\right)\right|_{Y-V}=0 .
$$

Along with the exactness of the bottom row, this implies that

$$
\alpha_{\#}-q_{\varphi}^{*} \alpha_{\cup} \in\left\{\delta_{\varphi}^{*}\left(\beta_{S V}\right): \beta_{S V} \in H^{m-1}(S V ; \mathbb{Z})\right\} .
$$

The claim then follows from the observation that

$$
\left\{\delta_{\varphi}^{*}\left(\beta_{S V}\right): \beta_{S V} \in H^{m-1}(S V ; \mathbb{Z})\right\} \subset\left\{q_{\varphi}^{*}\left(\alpha_{\cup}\right): \alpha_{\cup} \in H^{m}\left(X \cup_{V} Y ; \mathbb{Z}\right)\right\},
$$


which is established below.

Choose an open subset $\widetilde{Y}$ of $X \cup_{V} Y$ consisting of $Y$ and a tubular neighborhood of $V$ in $X$. Let $\mathcal{S}_{\cup}^{*}$ denote the cochain complex of $\mathbb{Z}$-valued homomorphisms on the sub-complex of singular chains generated by simplicies in $X \cup_{V} Y$ with images in either $X-V$ or $\widetilde{Y}$. Similarly, let $\mathcal{S}_{\#}^{*}$ denote the cochain complex of $\mathbb{Z}$-valued homomorphisms on the sub-complex of singular chains generated by simplicies in $X \#_{\varphi} Y$ with images in either $q_{\varphi}^{-1}(X-V)$ or $q_{\varphi}^{-1}(\widetilde{Y})$. By [20, Section 5.32], the restriction homomorphisms from the usual singular cochain complexes,

$$
\mathcal{S}^{*}\left(X \cup_{V} Y\right) \longrightarrow \mathcal{S}_{\cup}^{*} \quad \text { and } \quad \mathcal{S}^{*}\left(X \#{ }_{\varphi} Y\right) \longrightarrow \mathcal{S}_{\#}^{*},
$$

induce isomorphisms in cohomology. Thus, we can replace the domains of these homomorphisms by their targets in order to verify (4.23). Let $V_{\cup}=(X-V) \cap \widetilde{Y}$ and $S V_{\#}=q_{\varphi}^{-1}\left(V_{\cup}\right)$.

For any $\eta \in \mathcal{S}^{*}\left(S V_{\#}\right)$, define

$$
\begin{aligned}
\eta_{q_{\varphi}^{-1}(X-V)} & \in \mathcal{S}^{*}\left(q_{\varphi}^{-1}(X-V)\right), \quad \eta_{q_{\varphi}^{-1}(X-V)}(\sigma)= \begin{cases}\eta(\sigma), & \text { if } \operatorname{Im} \sigma \subset S V_{\#} ; \\
0, & \text { otherwise; }\end{cases} \\
\eta_{\#} \in \mathcal{S}_{\#}^{*}, & \eta_{\#}(\sigma)= \begin{cases}\eta_{q_{\varphi}^{-1}(X-V)}(\partial \sigma), & \text { if } \operatorname{Im} \sigma \subset q_{\varphi}^{-1}(X-V) ; \\
0, & \text { if } \operatorname{Im} \sigma \subset q_{\varphi}^{-1}(\widetilde{Y}) ;\end{cases} \\
\eta_{\cup} \in \mathcal{S}_{\cup}^{*}, & \eta_{\cup}(\sigma)= \begin{cases}\eta_{q_{\varphi}^{-1}(X-V)}\left(\partial\left(q_{\varphi}^{-1} \circ \sigma\right)\right), & \text { if } \operatorname{Im} \sigma \subset X-V ; \\
0, & \text { if } \operatorname{Im} \sigma \widetilde{Y} ;\end{cases}
\end{aligned}
$$

where $\sigma$ denotes an appropriate singular simplex. The homomorphisms $\eta_{\#}$ and $\eta_{\cup}$ are well-defined on the overlaps if $\delta \eta=0$, i.e. $\eta$ determines an element $[\eta]$ in $H^{*}\left(S V_{\#}\right)$. In such a case,

$$
\delta_{\varphi}^{*}[\eta]=\left[\eta_{\#}\right], \quad q_{\varphi}^{*}\left[\eta_{\cup}\right]=\left[\eta_{\#}\right],
$$

by the construction of the connecting homomorphism in the Snake Lemma and the definition of pull-back homomorphisms. This establishes (4.23).

In [8, Section 13], the cohomology classes on $X \#_{\varphi} Y$ not contained in the left-hand side of (4.20) are described as cutting through neck, i.e. $S V \subset X \#_{\varphi} Y$. The next statement makes this terminology precise.

Corollary 4.12. Suppose $X, Y, V, \varphi$, and $q_{\varphi}$ are as in Lemma 4.11, $X$ and $Y$ are compact, $\mathfrak{c}$ is the codimension of $V$ in $X$ and $Y$, and $\alpha_{\#} \in H^{*}\left(X \#_{\varphi} Y ; \mathbb{Z}\right)$. Then, $\alpha_{\#}=q_{\varphi}^{*} \alpha_{\cup}$ for some $\alpha_{\cup} \in H^{*}\left(X \cup_{V} Y ; \mathbb{Z}\right)$ if and only if $\mathrm{PD}_{X \#_{\varphi} Y}\left(\alpha_{\#}\right)$ can be represented by a pseudocycle $f_{\#}: Z_{\#} \longrightarrow X \#_{\varphi} Y$ transverse to $S V$ such that $f_{\#}^{-1}(S V)=f_{V}^{*} S V$ for some pseudocycle $f_{V}: Z_{V} \longrightarrow V$ of dimension $\mathfrak{c}$ less.

Proof. Let $f_{\#}: Z_{\#} \longrightarrow X \#{ }_{\varphi} Y$ be a pseudocycle representative for the Poincare dual of $\alpha_{\#}$ transverse to $S V$. The restriction of $f_{\#}$ to $f_{\#}^{-1}(S V)$ then represents the Poincare dual of $\left.\alpha_{\#}\right|_{S V}$.

(1) If $f_{\#}^{-1}(S V)=f_{V}^{*} S V$ for some pseudocycle $f_{V}: Z_{V} \longrightarrow V$ of dimension $\mathfrak{c}$ less, $\left.\alpha_{\#}\right|_{S V}=q_{V}^{*} \alpha_{V}$, where $\alpha_{V} \in H^{*}(V ; \mathbb{Z})$ is the Poincare dual of the class represented by $f_{V}$. Lemma 4.11 then implies 
that $\alpha_{\#}=q_{\varphi}^{*} \alpha_{\cup}$ for some $\alpha_{\cup} \in H^{*}\left(X \cup_{V} Y ; \mathbb{Z}\right)$.

(2) If $\alpha_{\#}=q_{\varphi}^{*} \alpha_{\cup}$ for some $\alpha_{\cup} \in H^{*}\left(X \cup_{V} Y\right.$; $\left.\mathbb{Z}\right)$, then $\left.\alpha_{\#}\right|_{S V}=q_{V}^{*} \alpha_{V}$ for some $\alpha_{V} \in H^{*}(V ; \mathbb{Z})$; see Lemma 4.11, Let $f_{V}: Z_{V} \longrightarrow V$ be a pseudocycle representing the Poincare dual of $\alpha_{V}$ and $\widetilde{f}_{V}: f_{V}^{*} S V \longrightarrow S V$ be the induced pseudocycle from the total space of the bundle $S V \longrightarrow V$ pulled back by $f_{V}$; see the end of Section 3.1 . Thus, there exists a pseudocycle equivalence $\tilde{f}: \widetilde{Z} \longrightarrow S V$ so that

$$
\partial \widetilde{f}=\left.f_{\#}\right|_{f_{\#}^{-1}\left(S_{V}\right)}-\widetilde{f}_{V} .
$$

Cutting $Z_{\#}$ along the hypersurface $f_{\#}^{-1}(S V)$, gluing in $\tilde{f}$ and $-\widetilde{f}$ along the resulting cuts, identifying $\widetilde{f}$ and $-\widetilde{f}$ along $\widetilde{f}_{V}$, and moving $\pm \widetilde{f}$ on the complement of $\widetilde{f}_{V}$ outside $S V$, we obtain a pseudocycle representative $\widehat{f}_{\#}: \widehat{Z}_{\#} \longrightarrow X \#_{\varphi} Y$ for the Poincare dual of $\alpha_{\#}$ transverse to $S V$ such that $\widehat{f}_{\#}^{-1}(S V)=f_{V}^{*} S V$.

Remark 4.13. There is a slight misstatement in part (a) at the bottom of page 996 in [8] related to the $\mathfrak{c}=2$ case of Lemma 4.11, since the first map in [ $\underline{8},(10.13)]$ is never injective for dimensional reasons. The statement in (a) should instead be that $\alpha \in H^{m}\left(Z_{\lambda} ; \mathbb{Z}\right)$ separates if

$$
\cup c_{1}: H^{m-1}(V ; \mathbb{Z}) \longrightarrow H^{m+1}(V ; \mathbb{Z})
$$

is injective. In (b), $j^{*}: H^{*}\left(Z_{\lambda} ; \mathbb{Z}\right) \longrightarrow H^{*}(S V ; \mathbb{Z})$ is the restriction map.

\section{$5 \quad$ Abelian covers of topological spaces}

The notation for the abelian covers of topological spaces relevant in our context is introduced in Section 5.1. Section 5.2 is concerned with their topological properties, focusing on whether their (co)homology is finitely generated or not.

By a topological space $V$, we will mean a locally path-connected and a semilocally simply connected topological space $V$, as in [16, $§ 25,82]$; all manifolds and more generally CW-complexes fall in this category. The first assumption implies that the connected and path-connected components of $V$ are the same; see [16, Theorem 25.5]. The two assumptions together imply the connected covers of the connected components $V_{r}$ of $V$ are classified by their fundamental subgroups $\pi_{1}\left(V_{r}\right)$; see [16, Theorems 79.4, 82.1].

\subsection{Notation and examples}

Let $\mathbb{Z}_{ \pm} \subset \mathbb{Z}$ denote the nonzero integers. For a tuple $\mathbf{s}=\left(s_{1}, \ldots, s_{\ell}\right) \in \mathbb{Z}_{ \pm}^{\ell}$ with $\ell \in \mathbb{Z}^{\geq 0}$, we denote by $\operatorname{gcd}(\mathbf{s})$ the greatest common divisor of $s_{1}, \ldots, s_{\ell}$; if $\ell=0$, we set $\operatorname{gcd}(\mathbf{s})=0$.

Let $V$ be a topological space. For any submodule $H \subset H_{1}(V ; \mathbb{Z})$, let

$$
q_{H}: H_{1}(V ; \mathbb{Z}) \longrightarrow \mathcal{R}_{H} \equiv \frac{H_{1}(V ; \mathbb{Z})}{H}
$$

be the projection to the corresponding quotient module. If $V_{1}, \ldots, V_{N}$ are the topological components of $V, \ell_{1}, \ldots, \ell_{N} \in \mathbb{Z}^{\geq 0}$, and $\mathbf{s}_{1} \in \mathbb{Z}_{ \pm}^{\ell_{1}}, \ldots, \mathbf{s}_{N} \in \mathbb{Z}_{ \pm}^{\ell_{N}}$, then the topological space

$$
V_{\mathbf{s}_{1} \ldots \mathbf{s}_{N}} \equiv V_{1}^{\ell_{1}} \times \ldots \times V_{N}^{\ell_{N}}
$$


is connected.

With $V$ and $\mathbf{s}_{1}, \ldots, \mathbf{s}_{N}$ as above, define

$$
\begin{gathered}
\Phi_{V ; \mathbf{s}_{1} \ldots \mathbf{s}_{N}}: H_{1}\left(V_{\mathbf{s}_{1} \ldots \mathbf{s}_{N}} ; \mathbb{Z}\right)=\bigoplus_{r=1}^{N} H_{1}\left(V_{r} ; \mathbb{Z}\right)^{\oplus \ell_{r}} \longrightarrow H_{1}(V ; \mathbb{Z}), \\
\Phi_{V ; \mathbf{s}_{1} \ldots \mathbf{s}_{N}}\left(\left(\gamma_{r ; i}\right)_{i \leq \ell_{r}, r \leq N}\right)=\sum_{r=1}^{N} \sum_{i=1}^{\ell_{r}} s_{r ; i} \gamma_{r ; i} .
\end{gathered}
$$

For any submodule $H \subset H_{1}(V ; \mathbb{Z})$, let

$$
\begin{gathered}
H_{\mathbf{s}_{1} \ldots \mathbf{s}_{N}}=\Phi_{V ; \mathbf{s}_{1} \ldots \mathbf{s}_{N}}^{-1}(H) \subset H_{1}\left(V_{\mathbf{s}_{1} \ldots \mathbf{s}_{N}} ; \mathbb{Z}\right) \\
\mathcal{R}_{H ; \mathbf{s}_{1} \ldots \mathbf{s}_{N}}^{\prime}=\operatorname{Im}\left\{q_{H} \circ \Phi_{V ; \mathbf{s}_{1} \ldots \mathbf{s}_{N}}\right\} \subset \mathcal{R}_{H}, \quad \mathcal{R}_{H ; \mathbf{s}_{1} \ldots \mathbf{s}_{N}}=\frac{\mathcal{R}_{H}}{\mathcal{R}_{H ; \mathbf{s}_{1} \ldots \mathbf{s}_{N}}^{\prime}} \times \mathcal{R}_{H ; \mathbf{s}_{1} \ldots \mathbf{s}_{N}}^{\prime}
\end{gathered}
$$

If $\operatorname{gcd}\left(\mathbf{s}_{r}\right)=1$ for every $r=1, \ldots, N$, then

$$
\mathcal{R}_{H ; \mathbf{s}_{1} \ldots \mathbf{s}_{N}}^{\prime}=\mathcal{R}_{H ; \mathbf{s}_{1} \ldots \mathbf{s}_{N}}=\mathcal{R}_{H}
$$

If $V$ is connected, then $\mathcal{R}_{H ; \mathbf{s}}^{\prime}=\operatorname{gcd}(\mathbf{s}) \mathcal{R}_{H}$ for any $\mathbf{s} \in \mathbb{Z}_{ \pm}^{\ell}$ and $H_{(1)}=H$.

For each $r=1, \ldots, N$, let $\widehat{V}_{r} \longrightarrow V_{r}$ be the maximal abelian cover of $V_{r}$, i.e. the covering projection corresponding to the commutator subgroup of $\pi_{1}(V)$. The group of deck transformations of this regular covering is $H_{1}\left(V_{r} ; \mathbb{Z}\right)$. The maximal abelian cover of $V_{\mathbf{s}_{1} \ldots \mathbf{s}_{N}}$ is given by

$$
\widehat{V}_{\mathbf{s}_{1} \ldots \mathbf{s}_{N}} \equiv \prod_{r=1}^{N} \widehat{V}_{r}^{\ell_{r}} \longrightarrow V_{\mathbf{s}_{1} \ldots \mathbf{s}_{N}}
$$

there is a natural action of $H_{1}\left(V_{\mathbf{s}} ; \mathbb{Z}\right)$ on this space. For any submodule $H \subset H_{1}(V ; \mathbb{Z})$, let

$$
\begin{aligned}
& \pi_{H ; \mathbf{s}_{1} \ldots \mathbf{s}_{N}}^{\prime}: \widehat{V}_{H ; \mathbf{s}_{1} \ldots \mathbf{s}_{N}}^{\prime} \equiv \widehat{V}_{\mathbf{s}_{1} \ldots \mathbf{s}_{N}} / H_{\mathbf{s}_{1} \ldots \mathbf{s}_{N}} \longrightarrow V_{\mathbf{s}_{1} \ldots \mathbf{s}_{N}} \\
& \pi_{H ; \mathbf{s}_{1} \ldots \mathbf{s}_{N}}: \widehat{V}_{H ; \mathbf{s}_{1} \ldots \mathbf{s}_{N}} \equiv \frac{\mathcal{R}_{H}}{\mathcal{R}_{H ; \mathbf{s}_{1} \ldots \mathbf{s}_{N}}^{\prime}} \times \widehat{V}_{H ; \mathbf{s}_{1} \ldots \mathbf{s}_{N}}^{\prime} \longrightarrow V_{\mathbf{s}_{1} \ldots \mathbf{s}_{N}}
\end{aligned}
$$

We will write elements of the second covering as

$$
\left([\gamma]_{H ; \mathbf{s}_{1} \ldots \mathbf{s}_{N}},[\widehat{x}]_{H}\right) \in \frac{\mathcal{R}_{H}}{\mathcal{R}_{H ; \mathbf{s}_{1} \ldots \mathbf{s}_{N}}^{\prime}} \times \widehat{V}_{H ; \mathbf{s}_{1} \ldots \mathbf{s}_{N}}^{\prime},
$$

with the first component denoting the image of $\gamma \in H_{1}(V ; \mathbb{Z})$ under the homomorphism

$$
H_{1}(V ; \mathbb{Z}) \longrightarrow \mathcal{R}_{H} \longrightarrow \frac{\mathcal{R}_{H}}{\mathcal{R}_{H ; \mathbf{s}_{1} \ldots \mathbf{s}_{N}}^{\prime}}
$$

and the second component denoting the image of $\widehat{x} \in \widehat{V}_{\mathbf{s}_{1} \ldots \mathbf{s}_{N}}$. 
The groups of deck transformations of these regular coverings are

$$
\operatorname{Deck}\left(\pi_{H ; \mathbf{s}_{1} \ldots \mathbf{s}_{N}}^{\prime}\right)=\mathcal{R}_{H ; \mathbf{s}_{1} \ldots \mathbf{s}_{N}}^{\prime} \quad \text { and } \quad \operatorname{Deck}\left(\pi_{H ; \mathbf{s}_{1} \ldots \mathbf{s}_{N}}\right)=\mathcal{R}_{H ; \mathbf{s}_{1} \ldots \mathbf{s}_{N}},
$$

respectively. The action of $\mathcal{R}_{H ; \mathbf{s}_{1} \ldots \mathbf{s}_{N}}^{\prime}$ is induced from the default action of $H_{1}\left(V_{\mathbf{s}} ; \mathbb{Z}\right)$ on $\widehat{V}_{\mathbf{s}}$ via the surjective homomorphism

$$
q_{H} \circ \Phi_{V ; \mathbf{s}_{1} \ldots \mathbf{s}_{N}}: H_{1}\left(V_{\mathbf{s}} ; \mathbb{Z}\right) \longrightarrow \mathcal{R}_{H ; \mathbf{s}_{1} \ldots \mathbf{s}_{N}}^{\prime} ;
$$

the kernel of this homomorphism, i.e. $H_{\mathbf{s}_{1} \ldots \mathbf{s}_{N}}$, acts trivially on $\widehat{V}_{H ; \mathbf{s}_{1} \ldots \mathbf{s}_{N}}^{\prime}$.

If $V$ is connected, then

$$
\pi_{H} \equiv \pi_{H ;(1)}: \widehat{V}_{H} \equiv \widehat{V}_{H ;(1)}=\widehat{V} / H \longrightarrow V
$$

is the abelian covering corresponding to the subgroup $H \subset H_{1}(V ; \mathbb{Z})$, i.e. the covering corresponding to the normal subgroup $\operatorname{Hur}^{-1}(H) \subset \pi_{1}(V)$, where

$$
\text { Hur: } \pi_{1}\left(V_{\mathbf{s}_{1} \ldots \mathbf{s}_{N}}\right) \longrightarrow H_{1}\left(V_{\mathbf{s}_{1} \ldots \mathbf{s}_{N}} ; \mathbb{Z}\right)
$$

is Hurewicz homomorphism; see [19, Section 7.4].

A collection $\left\{\gamma_{j}\right\} \subset H_{1}(V ; \mathbb{Z})$ of representatives for the elements of $\mathcal{R}_{H} / \mathcal{R}_{H ; \mathbf{s}_{1} \ldots \mathbf{s}_{N}}^{\prime}$ induces a homomorphism

$$
H_{1}(V ; \mathbb{Z}) \longrightarrow \operatorname{Deck}\left(\pi_{H ; \mathbf{s}_{1} \ldots \mathbf{s}_{N}}\right), \quad \eta \longrightarrow \Theta_{\eta},
$$

as follows. For every $\eta \in H_{1}(V ; \mathbb{Z})$ and a coset representative $\gamma_{j}$, let $\gamma_{j}(\eta)$ be the unique coset representative from the chosen collection such that

$$
\gamma_{j}+\eta-\gamma_{j}(\eta)-\Phi_{V ; \mathbf{s}_{1} \ldots \mathbf{s}_{N}}\left(\eta_{j}\right) \in H
$$

for some $\eta_{j} \in H_{1}\left(V_{\mathbf{s}_{1} \ldots \mathbf{s}_{N}} ; \mathbb{Z}\right)$. Define

$$
\Theta_{\eta}: \widehat{V}_{H ; \mathbf{s}_{1} \ldots \mathbf{s}_{N}} \longrightarrow \widehat{V}_{H ; \mathbf{s}_{1} \ldots \mathbf{s}_{N}}, \quad \Theta_{\eta}\left(\left[\gamma_{j}\right]_{H ; \mathbf{s}_{1} \ldots \mathbf{s}_{N}},[\widehat{x}]_{H}\right)=\left(\left[\gamma_{j}(\eta)\right]_{H ; \mathbf{s}_{1} \ldots \mathbf{s}_{N}},\left[\eta_{j} \cdot \widehat{x}\right]_{H}\right) .
$$

Since (5.9) determines $\eta_{j}$ up to an element of $H_{\mathbf{s}_{1} \ldots \mathbf{s}_{N}}$, the last component of $\Theta_{\eta}$ is well-defined.

Suppose in addition that $V^{\prime} \subset V$ is the union of $V_{1}, \ldots, V_{N^{\prime}}$ for some $N^{\prime} \leq N$ and $H^{\prime} \subset H_{1}\left(V^{\prime} ; \mathbb{Z}\right)$ is a submodule. Let

$$
\begin{array}{ll}
q: V_{\mathbf{s}_{1} \ldots \mathbf{s}_{N}} \longrightarrow V_{\mathbf{s}_{1} \ldots \mathbf{s}_{N^{\prime}}}^{\prime}, & \left(x_{r ; i}\right)_{i \leq \ell_{r}, r \leq N} \longrightarrow\left(x_{r ; i}\right)_{i \leq \ell_{r}, r \leq N^{\prime}}, \\
\widehat{q}: \widehat{V}_{\mathbf{s}_{1} \ldots \mathbf{s}_{N}} \longrightarrow \widehat{V}_{\mathbf{s}_{1} \ldots \mathbf{s}_{N^{\prime}}}^{\prime}, & \left(\widehat{x}_{r ; i}\right)_{i \leq \ell_{r}, r \leq N} \longrightarrow\left(\widehat{x}_{r ; i}\right)_{i \leq \ell_{r}, r \leq N^{\prime}}
\end{array}
$$

denote the projections to the $V^{\prime}$ - and $\widehat{V^{\prime}}$-components. If $H^{\prime}$ contains the image of $H$ under the projection

$$
q_{*}: H_{1}(V ; \mathbb{Z})=\bigoplus_{r=1}^{N} H_{1}\left(V_{r} ; \mathbb{Z}\right) \longrightarrow H_{1}\left(V^{\prime} ; \mathbb{Z}\right)=\bigoplus_{r=1}^{N^{\prime}} H_{1}\left(V_{r} ; \mathbb{Z}\right),
$$


then $q_{*}$ induces a commutative diagram of homomorphisms (not exact sequences)

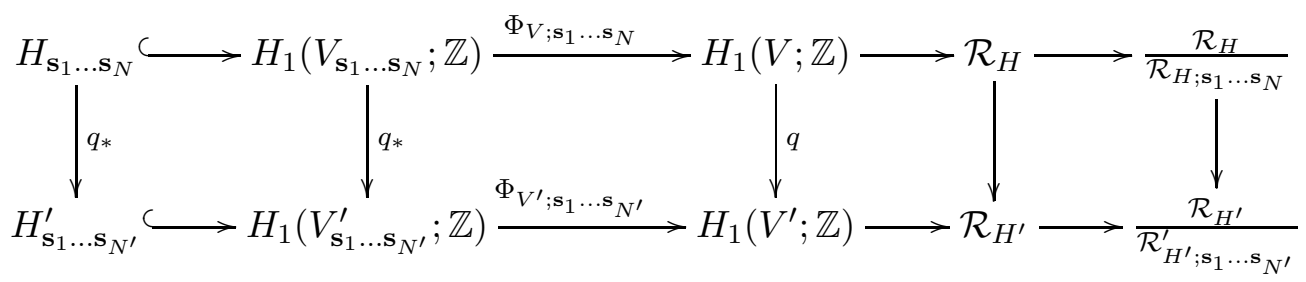

The continuous map

$$
\left([\gamma]_{H ; \mathbf{s}_{1} \ldots \mathbf{s}_{N}},[\widehat{x}]_{H}\right) \longrightarrow\left(\left[q_{*}(\gamma)\right]_{H^{\prime} ; \mathbf{s}_{1} \ldots \mathbf{s}_{N^{\prime}}},[\widehat{q}(\widehat{x})]_{H^{\prime}}\right)
$$

then induces a commutative diagram

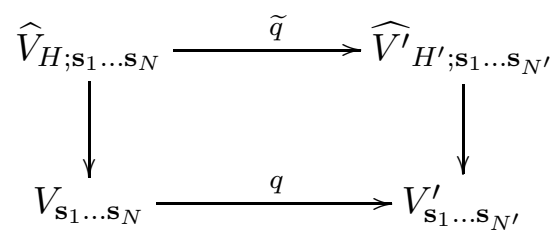

of fiber bundles.

Example 5.1. If $V=\mathbb{T}^{2}, \ell \in \mathbb{Z}^{+}$, and $H=\{0\}$, then

$$
\widehat{V}_{H ; \mathbf{s}}=\mathbb{C} \times \mathbb{T}_{\mathbf{s}}^{2(\ell-1)}, \quad \text { where } \quad \mathbb{T}_{\mathbf{s}}^{2(\ell-1)}=\left\{\left(z_{i}\right)_{i \leq \ell} \in \mathbb{C}^{\ell}: \sum_{i=1}^{\ell} s_{i} z_{i} \in \mathbb{Z} \oplus \mathbf{i} \mathbb{Z}\right\} / \mathbb{Z}^{2 \ell} \subset \mathbb{T}^{2 \ell}=V_{\mathbf{s}}
$$

The second covering in (5.6) can be written as

$$
\mathbb{C} \times \mathbb{T}_{\mathbf{s}}^{2(\ell-1)} \longrightarrow \mathbb{T}^{2 \ell}, \quad\left(z,\left[z_{i}\right]_{i \leq \ell}\right) \longrightarrow\left[z_{i}-\frac{z}{s_{i}}\right]_{i \leq \ell}
$$

A path $t \longrightarrow\left[\gamma_{i^{\prime}}(t)\right]_{i^{\prime} \leq \ell}$ in $\mathbb{T}^{2 \ell}$ lifts to the path

$$
t \longrightarrow\left(\frac{1}{\ell} \sum_{i^{\prime}=1}^{\ell} s_{i^{\prime}} \gamma_{i^{\prime}}(t),\left[\gamma_{i}(t)+\frac{1}{\ell s_{i}} \sum_{i^{\prime}=1}^{\ell} s_{i^{\prime}} \gamma_{i^{\prime}}(t)\right]_{i \leq \ell}\right)
$$

in $\mathbb{C} \times \mathbb{T}_{\mathbf{S}}^{2(\ell-1)}$. Under the standard identification of $H_{1}\left(\mathbb{T}^{2} ; \mathbb{Z}\right)$ with $\mathbb{Z} \oplus \mathbf{i} \mathbb{Z}$, the action of $H_{1}\left(\mathbb{T}^{2} ; \mathbb{Z}\right)^{\oplus \ell}$ on this cover is thus given by

$$
\left(\gamma_{i^{\prime}}\right)_{i^{\prime} \leq \ell} \cdot\left(z,\left[z_{i}\right]_{i \leq \ell}\right)=\left(z+\frac{1}{\ell} \sum_{i^{\prime}=1}^{\ell} s_{i^{\prime}} \gamma_{i^{\prime}},\left[z_{i}+\frac{1}{\ell s_{i}} \sum_{i^{\prime}=1}^{\ell} s_{i^{\prime}} \gamma_{i^{\prime}}\right]_{i \leq \ell}\right) .
$$

The group of deck transformations of this cover is $\mathbb{Z}_{\operatorname{gcd}(\mathbf{s})}^{2} \oplus \operatorname{gcd}(\mathbf{s}) \mathbb{Z}^{2}$. The action of the second component is induced by the action of $H_{1}\left(\mathbb{T}^{2} ; \mathbb{Z}\right)^{\oplus \ell}$ via the surjective homomorphism

$$
H_{1}\left(\mathbb{T}^{2} ; \mathbb{Z}\right)^{\oplus \ell} \longrightarrow \operatorname{gcd}(\mathbf{s}) H_{1}\left(\mathbb{T}^{2} ; \mathbb{Z}\right), \quad\left(\gamma_{i^{\prime}}\right)_{i^{\prime} \leq \ell} \longrightarrow \sum_{i^{\prime}=1}^{\ell} s_{i^{\prime}} \gamma_{i^{\prime}}
$$




\subsection{Some properties}

We now describe some cases when the (co)homology of the abelian covers $\widehat{V}_{H ; \mathbf{s}}$ is finitely generated. As indicated in [4, Sections 1.2,1.3], the refinement to the usual GW-invariants suggested in [7] is more likely to lead to qualitative applications in the symplectic sum context in such cases. We continue with the notation of Section 5.1 .

Lemma 5.2. Let $V$ be a finite connected $C W$-complex, $H \subset H_{1}(V ; \mathbb{Z})$ be a submodule, and $\mathbf{s} \in \mathbb{Z}_{ \pm}^{\ell}$ with $\ell \in \mathbb{Z}^{+}$. If $H_{*}\left(\widehat{V}_{H} ; \mathbb{Q}\right)$ is finitely generated, then so is $H_{*}\left(\widehat{V}_{H ; \mathbf{s}} ; \mathbb{Q}\right)$.

Proof. Since $\mathcal{R}_{H} / \mathcal{R}_{H ; \mathbf{s}}^{\prime}$ is finite, it is sufficient to show that $H_{*}\left(\widehat{V}_{H ; \mathbf{s}}^{\prime} ; \mathbb{Q}\right)$ is finitely generated. By the Universal Coefficient Theorem [17, Theorem 53.5], $H_{*}$ is finitely generated if and only if $H^{*}$ is. Since $H_{\mathbf{s} / \operatorname{gcd}(\mathbf{s})} \subset H_{\mathbf{s}}$ and $H \subset H_{1}(V ; \mathbb{Z})$ is finitely generated,

$$
q: \widehat{V}_{H ; \mathbf{s} / \operatorname{gcd}(\mathbf{s})}^{\prime}=\widehat{V}^{\ell} / H_{\mathbf{s} / \operatorname{gcd}(\mathbf{s})} \longrightarrow \widehat{V}_{H ; \mathbf{s}}^{\prime}=\widehat{V}^{\ell} / H_{\mathbf{s}}
$$

is a finite covering and so the homomorphism

$$
q^{*}: H^{*}\left(\widehat{V}_{H ; \mathbf{s}}^{\prime} ; \mathbb{Q}\right) \longrightarrow H^{*}\left(\widehat{V}_{H ; \mathbf{s} / \operatorname{gcd}(\mathbf{s})}^{\prime} ; \mathbb{Q}\right)
$$

is injective. In particular, the claim of the lemma holds if $\ell=1$.

Suppose $\ell>1$. Let $\mathbf{s}^{\prime}$ denote the tuple consisting of the first $(\ell-1)$ components of $\mathbf{s}$ and

$$
H^{\prime}=H+s_{\ell} H_{1}(V ; \mathbb{Z}) \subset H_{1}(V ; \mathbb{Z}) .
$$

The projection $\widehat{V}^{\ell} \longrightarrow V$ onto the last component induces a fiber bundle

$$
q_{\ell}: \widehat{V}_{H ; \mathbf{s}}^{\prime}=\widehat{V}^{\ell} / H_{\mathbf{s}} \longrightarrow V=\widehat{V} / H_{1}(V ; \mathbb{Z})
$$

with fiber $\widehat{V}_{H^{\prime} ; \mathbf{s}^{\prime}}$. By Serre's Spectral Sequence (e.g. Theorem 9.2.1, 9.2.17, or 9.3.1 in [19] applied with $\mathbb{Z}_{2}$-coefficients in the last two cases), $H^{*}\left(\widehat{V}_{H ; \mathbf{s}}^{\prime} ; \mathbb{Q}\right)$ is thus finitely generated if $H^{*}(V ; \mathbb{Q})$ and $H^{*}\left(\widehat{V}_{H^{\prime} ; \mathbf{s}^{\prime}}^{\prime} ; \mathbb{Q}\right)$ are finitely generated. This is the case for $H^{*}(V ; \mathbb{Q})$ because $V$ is a finite $\mathrm{CW}$ complex. By induction on $\ell$, we can assume that this is also the case for $H^{*}\left(\widehat{V}_{H^{\prime} ; \mathbf{s}^{\prime}}^{\prime} ; \mathbb{Q}\right)$.

Remark 5.3. The statement and proof of Lemma 5.2 can be adapted to a disconnected $V$. For each $r=1, \ldots, N$, let

$$
\mathcal{R}_{H ; r}=q_{H}\left(H_{1}\left(V_{r} ; \mathbb{Z}\right)\right) \subset \mathcal{R}_{H}
$$

these modules span $\mathcal{R}_{H}$. The first factor in the definition of $\widehat{V}_{H ; \mathbf{s}_{1} \ldots \mathbf{s}_{N}}$ in (5.6) is finite if and only if the submodule

$$
\widetilde{\mathcal{R}}_{H ; \mathbf{s}_{1} \ldots \mathbf{s}_{N}} \equiv \sum_{\substack{1 \leq r \leq N \\ \ell_{r} \neq 0}} \mathcal{R}_{H ; r} \subset \mathcal{R}_{H}
$$

has finite index. This index is finite if $\ell_{r} \neq 0$ whenever $H_{1}\left(V_{r} ; \mathbb{Q}\right) \neq\{0\}$ or if $V=\{0, \infty\} \times F$ for some connected $F$ and

$$
H=H_{\Delta} \subset H_{1}(V ; \mathbb{Z})=H_{1}(F ; \mathbb{Z}) \oplus H_{1}(F ; \mathbb{Z})
$$


is the diagonal. If $\widetilde{\mathcal{R}}_{H ; \mathbf{s}_{1} \ldots \mathbf{s}_{N}}$ does not have finite index in $\mathcal{R}_{H}$, then $H_{*}\left(\widehat{V}_{H ; \mathbf{s}_{1} \ldots \mathbf{s}_{N}} ; \mathbb{Q}\right)$ is clearly not finitely generated. If the index is finite, $H_{*}\left(\widehat{V}_{H ; \mathbf{s}_{1} \ldots \mathbf{s}_{N}} ; \mathbb{Q}\right)$ is finitely generated. For the purposes of establishing this statement, $V$ can be replaced by the union of $V_{r}$ with $\ell_{r} \neq 0$ and $H$ by its intersection with the $H_{1}$ of this subspace. Thus, we can assume that $\ell_{r} \neq 0$ for all $r=1, \ldots, N$. The proof of Lemma 5.2 then applies by projecting to $V_{N}$ and replacing $s_{\ell} H_{1}(V ; \mathbb{Z})$ in (5.13) by $s_{N ; \ell_{N}} H_{1}\left(V_{N} ; \mathbb{Z}\right)$ if $\ell_{N} \geq 2$ and by $H_{1}\left(V_{N} ; \mathbb{Z}\right)$ if $\ell_{N}=1$ (in this case, $V_{N}$ no longer appears in the fiber).

We next relate the action of $\operatorname{Deck}\left(\pi_{H ; \mathbf{s}_{1} \ldots \mathbf{s}_{N}}^{\prime}\right)$ on the cohomology of $\widehat{V}_{H ; \mathbf{s}_{1} \ldots \mathbf{s}_{N}}^{\prime}$ to the flux subgroup $\operatorname{Flux}(V) \subset H_{1}(V ; \mathbb{Z})$ defined in Section 1.2 . Let $V_{1}, \ldots, V_{N}$ be the connected components of $V$, $\mathbf{s}_{1} \in \mathbb{Z}_{ \pm}^{\ell_{1}}, \ldots, \mathbf{s}_{N} \in \mathbb{Z}_{ \pm}^{\ell_{N}}$, and $H \subset H_{1}(V ; \mathbb{Z})$ be a submodule. Define

$$
\begin{aligned}
\operatorname{Flux}(V)_{H ; \mathbf{s}_{1} \ldots \mathbf{s}_{N}} & =\left\{q_{H}\left(\Phi_{V ; \mathbf{s}_{1} \ldots \mathbf{s}_{N}}\left(\left(\gamma_{r ; i}\right)_{i \leq \ell_{r}, r \leq N}\right)\right): \gamma_{r ; i} \in \operatorname{Flux}\left(V_{r}\right) \forall i \leq \ell_{r}, r \leq N\right\} \\
& \subset \mathcal{R}_{H ; \mathbf{s}_{1} \ldots \mathbf{s}_{N}}^{\prime} \subset \mathcal{R}_{H} .
\end{aligned}
$$

Lemma 5.4. Let $V, V_{1}, \ldots, V_{N}, \mathbf{s}_{1}, \ldots, \mathbf{s}_{N}$, and $H$ be as above. If

$$
\gamma \in H_{1}\left(V_{\mathbf{s}_{1} \ldots \mathbf{s}_{N}} ; \mathbb{Z}\right) \quad \text { and } \quad q_{H}\left(\Phi_{V ; \mathbf{s}_{1} \ldots \mathbf{s}_{N}}(\gamma)\right) \in \operatorname{Flux}(V)_{H ; \mathbf{s}_{1} \ldots \mathbf{s}_{N}}
$$

then the isomorphism

$$
\{\gamma \cdot\}^{*}: H^{*}\left(\widehat{V}_{H ; \mathbf{s}_{1} \ldots \mathbf{s}_{N}}^{\prime} ; \mathbb{Z}\right) \longrightarrow H^{*}\left(\widehat{V}_{H ; \mathbf{s}_{1} \ldots \mathbf{s}_{N}}^{\prime} ; \mathbb{Z}\right)
$$

is the identity.

Proof. Let

$$
\gamma^{\prime} \equiv\left(\gamma_{r ; i}^{\prime}\right)_{i \leq \ell_{r}, r \leq N} \in \bigoplus_{r=1}^{N} \operatorname{Flux}\left(V_{r}\right)^{\oplus \ell_{r}}
$$

be such that $q_{H}\left(\Phi_{V ; \mathbf{s}_{1} \ldots \mathbf{s}_{N}}(\gamma)\right)=q_{H}\left(\Phi_{V ; \mathbf{s}_{1} \ldots \mathbf{s}_{N}}\left(\gamma^{\prime}\right)\right)$. Since $\gamma^{\prime}-\gamma \in H_{\mathbf{s}_{1} \ldots \mathbf{s}_{N}}$, the actions of $\gamma$ and $\gamma^{\prime}$ on $\widehat{V}_{H ; \mathbf{s}_{1} \ldots \mathbf{s}_{N}}^{\prime}$ are the same and we can assume that $\gamma^{\prime}=\gamma$.

For each $r=1, \ldots, N$ and $i=1, \ldots, \ell_{r}$, let $\Psi_{r ; i ; t}: V_{r} \longrightarrow V_{r}$ be a loop of homeomorphisms generating $\gamma_{r ; i}$ such that $\Psi_{r ; i ; 0}=$ id. These loops lift to paths of homeomorphisms

$$
\begin{array}{cll}
\widehat{\Psi}_{r ; i t t}: \widehat{V}_{r} \longrightarrow \widehat{V}_{r}, & t \in[0,1], & \widehat{\Psi}_{r ; i ; 0}=\mathrm{id}_{\widehat{V}_{r}}, \quad \widehat{\Psi}_{r ; i ; 1}\left(\widehat{x}_{r ; i}\right)=\gamma_{r ; i} \cdot \widehat{x}_{r ; i}, \\
\widetilde{\Psi}_{t}: \widehat{V}_{H ; \mathbf{s}_{1} \ldots \mathbf{s}_{N}}^{\prime} \longrightarrow \widehat{V}_{H ; \mathbf{s}_{1} \ldots \mathbf{s}_{N}}^{\prime}, & t \in[0,1], & \widetilde{\Psi}_{t}\left(\left[\left(\widehat{x}_{r ; i}\right)_{i \leq \ell_{r}, r \leq N}\right]_{H}\right)=\left[\left(\widehat{\Psi}_{r ; i t}\left(\widehat{x}_{r ; i}\right)\right)_{i \leq \ell_{r}, r \leq N}\right]_{H} .
\end{array}
$$

Since $\widetilde{\Psi}_{1}=\gamma \cdot$, the homeomorphism $\gamma \cdot$ of $\widehat{V}_{H ; \mathbf{s}_{1} \ldots \mathbf{s}_{N}}^{\prime}$ is homotopic to the identity. This implies the claim.

Corollary 5.5. Let $V$ be a finite $C W$-complex with connected components $V_{1}, \ldots, V_{N}, H \subset H_{1}(V ; \mathbb{Z})$ be a submodule, $\ell_{1}, \ldots \ell_{N} \in \mathbb{Z}^{+}$, and $\mathbf{s}_{1} \in \mathbb{Z}_{ \pm}^{\ell_{1}}, \ldots, \mathbf{s}_{N} \in \mathbb{Z}_{ \pm}^{\ell_{N}}$.

(1) If the index of $\operatorname{Flux}(V)_{H}$ in $\mathcal{R}_{H}$ is finite, then $H^{*}\left(\widehat{V}_{H ; \mathbf{s}_{1} \ldots \mathbf{s}_{N}}^{\prime} ; \mathbb{Q}\right)$ is finitely generated.

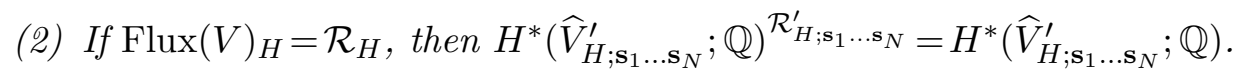

(3) If $\operatorname{Flux}(V)_{H}=\mathcal{R}_{H}$ and $\mathrm{rk}_{\mathbb{Z}} \mathcal{R}_{H} \leq 1$, then $\pi_{H ; \mathbf{s}_{1} \ldots \mathbf{s}_{N}}^{* *} H^{*}\left(V_{\mathbf{s}_{1} \ldots \mathbf{s}_{N}} ; \mathbb{Q}\right)=H^{*}\left(\widehat{V}_{H ; \mathbf{s}_{1} \ldots \mathbf{s}_{N}}^{\prime} ; \mathbb{Q}\right)$. 
Proof. $(1)$ The vector space $H^{*}\left(\widehat{V}_{H ; \mathbf{s}_{1} \ldots \mathbf{s}_{N}}^{\prime} ; \mathbb{Q}\right)$ is finitely generated over the group ring of

$$
\operatorname{Deck}\left(\pi_{H ; \mathbf{s}_{1} \ldots \mathbf{s}_{N}}^{\prime}\right)=\mathcal{R}_{H ; \mathbf{s}_{1} \ldots \mathbf{s}_{N}}^{\prime} .
$$

By Lemma 5.4, the elements of Flux $(V)_{H ; \mathbf{s}_{1} \ldots \mathbf{s}_{N}}$ act trivially on the cohomology of $\widehat{V}_{H ; \mathbf{s}_{1} \ldots \mathbf{s}_{N}}^{\prime}$. Thus, $H^{*}\left(\widehat{V}_{H ; \mathbf{s}_{1} \ldots \mathbf{s}_{N}}^{\prime} ; \mathbb{Q}\right)$ is finitely generated over the group ring of the quotient $\mathcal{R}_{H ; \mathbf{s}_{1} \ldots \mathbf{s}_{N}}^{\prime} / \operatorname{Flux}(V)_{H ; \mathbf{s}_{1} \ldots \mathbf{s}_{N}}$. If the index of $\operatorname{Flux}(V)_{H}$ in $\mathcal{R}_{H}$ is finite, then the index of $\operatorname{Flux}(V)_{H ; \mathbf{s}_{1} \ldots \mathbf{s}_{N}}$ in $\mathcal{R}_{H ; \mathbf{s}_{1} \ldots \mathbf{s}_{N}}^{\prime}$ is also finite and so $H^{*}\left(\widehat{V}_{H ; \mathbf{s}_{1} \ldots \mathbf{s}_{N}}^{\prime} ; \mathbb{Q}\right)$ is finitely generated over $\mathbb{Q}$.

(2) If $\operatorname{Flux}(V)_{H}=\mathcal{R}_{H}$, then $\operatorname{Flux}(V)_{H ; \mathbf{s}_{1} \ldots \mathbf{s}_{N}}=\mathcal{R}_{H ; \mathbf{s}_{1} \ldots \mathbf{s}_{N}}^{\prime}$. By Lemma [5.4, $\mathcal{R}_{H ; \mathbf{s}_{1} \ldots \mathbf{s}_{N}}^{\prime}$ thus acts trivially on $H^{*}\left(\widehat{V}_{H ; \mathbf{s}_{1} \ldots \mathbf{s}_{N}}^{\prime} ; \mathbb{Q}\right)$.

(3) The last claim of this corollary follows from the second claim and Corollary 5.8 below.

In the remainder of this section, we establish Lemmas 5.6 and 5.7 below. They imply Corollary 5.8, which is used in the proof of Corollary 5.5 above. The statement and proof of Lemma 5.6 are well-known. As pointed out by M. Wendt and D. Ruberman on MathOverflow, the statement of Lemma 5.7 can be obtained either from a spectral sequence applied to the Borel construction associated to the $\mathbb{Z}$-covering $\widetilde{V}$ or from the short exact sequence in the proof of [14, Assertion 5]. As we are not aware of any published reference for these statements, we include their proofs for the sake of completeness. In the proof of Lemma 5.7, we represent each $\mathbb{Z}$-invariant cohomology class on a regular $\mathbb{Z}$-covering by an explicit cohomology class on the associated Borel construction (the argument suggested by D. Ruberman is more efficient, but does not produce such a cocycle).

For a cochain complex $\left(C^{*}, \delta\right)$ with an action of a group $G$, let

$$
\left(C^{*}, \delta\right)^{G} \equiv\left(\left(C^{*}\right)^{G},\left.\delta\right|_{\left(C^{*}\right)^{G}}\right), \quad \text { where } \quad\left(C^{*}\right)^{G}=\left\{\eta \in G^{*}: g \cdot \eta=\eta \forall g \in G\right\},
$$

be the $G$-invariant subcomplex of $\left(C^{*}, \delta\right)$ and

$$
H^{*}\left(C^{*}, \delta\right)^{G} \equiv\left\{[\eta] \in H^{*}\left(C^{*}, \delta\right): g \cdot[\eta]=[\eta] \forall g \in G\right\}
$$

be the $G$-invariant part of the cohomology of $\left(C^{*}, \delta\right)$. The inclusion $\left(C^{*}, \delta\right)^{G}$ into $\left(C^{*}, \delta\right)$ induces a homomorphism

$$
H^{*}\left(\left(C^{*}, \delta\right)^{G}\right) \longrightarrow H^{*}\left(C^{*}, \delta\right)^{G} .
$$

Lemma 5.6. Let $\left(C^{*}, \delta\right)$ be a cochain complex over $\mathbb{Q}$ with an action of a group $G$. If $G$ is finite, then the homomorphism (5.16) is an isomorphism.

Proof. Let $\eta \in C^{*}$ be a cocycle such that $[\eta] \in H^{*}\left(C^{*}, \delta\right)^{G}$. Then, the cocycle

$$
\eta_{G} \equiv \frac{1}{|G|} \sum_{g \in G} g \cdot \eta \in\left(C^{*}\right)^{G}
$$

also represents $[\eta]$ and so the homomorphism (15.16) is surjective. If $\eta \in\left(C^{*}\right)^{G}$ is a cocycle such that $\eta=\delta \mu$ for some cochain $\mu \in C^{*}$, then $\eta=\delta \mu_{G}$ and so the homomorphism (5.16) is injective. 
Let $\widetilde{V}$ be a topological space with an action of a group $G$ and

$$
\pi: \widetilde{V} \longrightarrow V \equiv \widetilde{V} / G
$$

be the projection to the quotient. Since $\pi$ commutes with the group action,

$$
\pi^{*} H^{*}(V ; \mathbb{Q}) \subset H^{*}(\widetilde{V} ; \mathbb{Q})^{G} \equiv H^{*}\left(C^{*}(\widetilde{V} ; \mathbb{Q}), \delta\right)^{G}
$$

In some important cases, the above inclusion is an equality. If $\pi: \widetilde{V} \longrightarrow V$ is a regular covering, i.e. the group $G$ of its deck transformations acts transitively on the fibers, then $V=\widetilde{V} / G$. In such a case, every $G$-invariant cochain (resp. cycle) on $\widetilde{V}$ descends to a cochain (resp. cycle) on $V$, i.e.

$$
\pi^{*}\left(C^{*}(V ; \mathbb{Q}), \delta\right)=\left(C^{*}(\widetilde{V} ; \mathbb{Q}), \delta\right)^{G},
$$

provided the topological space $V$ is sufficiently nice and a suitable cohomology theory is used (so that all elements of $C_{*}(V ; \mathbb{Q})$ lie inside evenly covered open subsets of $V$ ). The inclusion in (5.17) is an equality if the homomorphism (5.16) with $C^{*}=C^{*}(\widetilde{V} ; \mathbb{Q})$ is surjective. The two statements below describe regular coverings for which this is the case. For the CW-complexes appearing in these statements, we use the (co)chain complexes generated by the cells of a CWstructure subordinate to the evenly covered open subsets of $V$ or of its pullback to $\widetilde{V}$. A regular covering $\pi: \widetilde{V} \longrightarrow V$ is called abelian if its group of deck transformations is abelian.

Lemma 5.7. Let $\pi: \widetilde{V} \longrightarrow V$ be an abelian covering of a connected $C W$-complex with the group of deck transformations $\mathbb{Z}$. Suppose $G$ is a finite group that acts on $\widetilde{V}$ so that its action commutes with the action of $\mathbb{Z}$ and thus descends to $V$. Then,

$$
\pi^{*}\left(H^{*}(V ; \mathbb{Q})^{G}\right)=H^{*}(\widetilde{V} ; \mathbb{Q})^{\mathbb{Z} \times G} .
$$

Proof. Let $u: \widetilde{V} \longrightarrow \widetilde{V}$ be a generator of the $\mathbb{Z}$-action and

$$
B_{\mathbb{Z}} \widetilde{V}=(\mathbb{R} \times \widetilde{V}) / \mathbb{Z}, \quad(t, x) \sim(t-1, u \cdot x),
$$

be the corresponding Borel construction for $\widetilde{V}$. Since the actions of $\mathbb{Z}$ and $G$ on $\widetilde{V}$ commute, the latter induces a $G$-action on $B_{\mathbb{Z}} \widetilde{V}$. Since $\mathbb{Z}$ acts freely on $\widetilde{V}$, the projection

$$
q: B_{\mathbb{Z}} \widetilde{V} \longrightarrow V, \quad q([t, x])=\pi(x) \equiv[x],
$$

is a $G$-equivariant homotopy equivalence. The composition of the inclusion $\iota: \widetilde{V} \longrightarrow B_{\mathbb{Z}} \widetilde{V}$ of a fiber for the fibration $B_{\mathbb{Z}} \widetilde{V} \longrightarrow S^{1}$ with $q$ is the covering $\pi: \widetilde{V} \longrightarrow V$. Thus, it is sufficient to show that the inclusion

$$
\iota^{*}\left(H^{*}\left(B_{\mathbb{Z}} \tilde{V} ; \mathbb{Q}\right)^{G}\right) \subset H^{*}(\tilde{V} ; \mathbb{Q})^{\mathbb{Z} \times G}
$$

is in fact an equality.

Let $\eta \in C^{k}(\widetilde{V} ; \mathbb{Q})$ be a cocycle such that $[\eta] \in H^{k}(\widetilde{V} ; \mathbb{Q})^{\mathbb{Z} \times G}$. Since

$$
\eta_{G} \equiv \frac{1}{|G|} \sum_{g \in G} g \cdot \eta \in C^{k}(\widetilde{V} ; \mathbb{Q})^{G}
$$


determines the same element of $H^{k}\left(B_{\mathbb{Z}} \widetilde{V} ; \mathbb{Q}\right)$, we can assume that $\eta \in C^{k}(\widetilde{V} ; \mathbb{Q})^{G}$. Thus,

$$
u^{*} \eta-\eta=\delta \mu \quad \text { for some } \quad \mu \in C^{k-1}(\widetilde{V} ; \mathbb{Q})^{G} .
$$

The chain groups $C_{k}(\tilde{V}, \mathbb{Q})$ and $C_{k-1}(\tilde{V}, \mathbb{Q})$ are freely generated by the simplices

$$
\left\{u^{s} \circ \sigma_{i}: s \in \mathbb{Z}, i\right\} \quad \text { and } \quad\left\{u^{s} \circ \tau_{j}: s \in \mathbb{Z}, j\right\}
$$

for some $k$-cells $\sigma_{i}$ and $(k-1)$-cells $\tau_{j}$ on $\widetilde{V}$. Define

$$
\widetilde{\eta} \in C^{k}(\mathbb{R} \times \widetilde{V} ; \mathbb{Q})^{\mathbb{Z} \times G} \quad \text { by } \quad \widetilde{\eta}\left(\{r\} \times u^{s} \circ \sigma_{i}\right)=\eta\left(u^{r+s} \circ \sigma_{i}\right), \quad \widetilde{\eta}\left([r, r+1] \times u^{s} \circ \tau_{j}\right)=\mu\left(u^{r+s} \circ \tau_{j}\right) .
$$

If $\sigma_{i}$ is a $k$-cell and $\varpi_{\ell}$ is a $(k+1)$-cell on $\widetilde{V}$, then

$$
\begin{aligned}
\widetilde{\eta}\left(\partial\left([r, r+1] \times u^{s} \circ \sigma_{i}\right)\right) & =\widetilde{\eta}\left(\{r+1\} \times u^{s} \circ \sigma_{i}\right)-\widetilde{\eta}\left(\{r\} \times u^{s} \circ \sigma_{i}\right)-\widetilde{\eta}\left([r, r+1] \times u^{s} \circ \partial \sigma_{i}\right) \\
& =\left\{u^{*} \eta\right\}\left(u^{r+s} \circ \sigma_{i}\right)-\eta\left(u^{r+s} \circ \sigma_{i}\right)-\partial \mu\left(u^{r+s} \circ \sigma_{i}\right)=0, \\
\widetilde{\eta}\left(\partial\left(\{r\} \times u^{s} \circ \varpi_{\ell}\right)\right) & =\widetilde{\eta}\left(\{r\} \times u^{s} \circ \partial \varpi_{\ell}\right)=\{\delta \eta\}\left(u^{r+s} \circ \varpi_{\ell}\right)=0 .
\end{aligned}
$$

Thus, $\widetilde{\eta}$ is a $\mathbb{Z} \times G$-invariant cocycle and so descends to a $G$-invariant cocycle on $B_{\mathbb{Z}} \widetilde{V}$. The latter restricts to $\eta$ along the fiber of $B_{\mathbb{Z}} \widetilde{V} \longrightarrow S^{1}$ over $0 \in S^{1}$.

Corollary 5.8. Let $\pi: \widetilde{V} \longrightarrow V$ be an abelian covering of a connected $C W$-complex and $G$ be its group of deck transformations. If $G$ is finitely generated and $\operatorname{rk}_{\mathbb{Z}} G \leq 1$, then

$$
\pi^{*} H^{*}(V ; \mathbb{Q})=H^{*}(\widetilde{V} ; \mathbb{Q})^{G} .
$$

Proof. By [1, Theorem 12.6.4],

$$
G \approx \mathbb{Z}^{r} \times G_{f}
$$

for some $r \in \mathbb{Z}^{\geq 0}$ and some finite (abelian) group $G_{f}$. If $r=0$, the claim follows from (5.18) and Lemma 5.6.

Suppose $r=1$. Let $\widetilde{V}_{f}=\widetilde{V} / \mathbb{Z}$ and

$$
\pi_{\mathbb{Z}}: \widetilde{V} \longrightarrow \widetilde{V}_{f} \quad \text { and } \quad \pi_{f}: \widetilde{V}_{f} \longrightarrow V
$$

be the quotient projection maps. Thus, $\pi=\pi_{f} \circ \pi_{\mathbb{Z}}$. By the $r=0$ case above and Lemma 5.7 ,

$$
\pi_{f}^{*} H^{*}(V ; \mathbb{Q})=H^{*}\left(\widetilde{V}_{f} ; \mathbb{Q}\right)^{G_{f}} \quad \text { and } \quad \pi_{\mathbb{Z}}^{*}\left(H^{*}\left(\widetilde{V}_{f} ; \mathbb{Q}\right)^{G_{f}}\right)=H^{*}(\widetilde{V} ; \mathbb{Q})^{G},
$$

respectively. Combining the two equations, we obtain the $r=1$ case of the claim.

\section{The refined relative GW-counts}

We now provide the details needed to refine the standard relative $\mathrm{GW}$-invariants of $(X, V, \omega)$, as suggested in [7, Section 5] and outlined in Section 1.1. The coverings (1.6) are special cases of the abelian covers described in Section 5.1 and are specified at the beginning of Section 6.1, In the remainder of Section 6.1, we show that the total relative evaluation morphisms (1.3) lift to the these coverings and establish Theorem 1.2, The lifts (1.8) are not unique, but can be chosen consistently; see Theorem 6.5 in Section 6.2. Theorem 1.1 is proved in Section 6.3. 


\subsection{The rim tori covers}

Let $X$ be a compact oriented manifold and $V \subset X$ be a compact oriented submanifold of codimension $\mathfrak{c}$ with topological components $V_{1}, \ldots, V_{N}$. With $H_{X}^{V} \subset H_{1}(V ; \mathbb{Z})$ as in (3.2),

$$
\mathcal{R}_{H_{X}^{V}} \equiv H_{1}(V ; \mathbb{Z})_{X} \approx \mathcal{R}_{X}^{V}
$$

see Corollary 3.2. For $\ell_{1}, \ldots, \ell_{N} \in \mathbb{Z}^{\geq 0}$ and $\mathbf{s}_{1} \in \mathbb{Z}_{ \pm}^{\ell_{1}}, \ldots, \mathbf{s}_{N} \in \mathbb{Z}_{ \pm}^{\ell_{N}}$, define

$$
\begin{gathered}
H_{X ; \mathbf{s}_{1} \ldots \mathbf{s}_{N}}^{V}=\left(H_{X}^{V}\right)_{\mathbf{s}_{1} \ldots \mathbf{s}_{N}} \subset H_{1}\left(V_{\mathbf{s}_{1} \ldots \mathbf{s}_{N}} ; \mathbb{Z}\right), \\
\mathcal{R}_{X ; \mathbf{s}_{1} \ldots \mathbf{s}_{N}}^{\prime V}=\mathcal{R}_{H_{X}^{V} ; \mathbf{s}_{1} \ldots \mathbf{s}_{N}}^{\prime} \subset H_{1}(V ; \mathbb{Z})_{X}, \quad \mathcal{R}_{X ; \mathbf{s}_{1} \ldots \mathbf{s}_{N}}^{V}=\mathcal{R}_{H_{X}^{V} ; \mathbf{s}_{1} \ldots \mathbf{s}_{N}} .
\end{gathered}
$$

The rim tori covers (1.6) are the abelian covers

$$
\begin{aligned}
& \pi_{X ; \mathbf{s}_{1} \ldots \mathbf{s}_{N}}^{\prime V} \equiv \pi_{H_{X}^{V} ; \mathbf{s}_{1} \ldots \mathbf{s}_{N}}^{\prime V}: \widehat{V}_{X ; \mathbf{s}_{1} \ldots \mathbf{s}_{N}}^{\prime} \equiv \widehat{V}_{H_{X}^{V} ; \mathbf{s}_{1} \ldots \mathbf{s}_{N}}^{\prime} \longrightarrow V_{\mathbf{s}_{1} \ldots \mathbf{s}_{N}}, \\
& \pi_{X ; \mathbf{s}_{1} \ldots \mathbf{s}_{N}}^{V} \equiv \pi_{H_{X}^{V} ; \mathbf{s}_{1} \ldots \mathbf{s}_{N}}^{V}: \widehat{V}_{X ; \mathbf{s}_{1} \ldots \mathbf{s}_{N}} \equiv \widehat{V}_{H_{X}^{V} ; \mathbf{s}_{1} \ldots \mathbf{s}_{N}} \longrightarrow V_{\mathbf{s}_{1} \ldots \mathbf{s}_{N}} .
\end{aligned}
$$

We will write elements of the second covering as

$$
\left([\gamma]_{X ; \mathbf{s}_{1} \ldots \mathbf{s}_{N}},[\widehat{x}]_{X}\right) \in \frac{\mathcal{R}}{\mathcal{R}_{X ; \mathbf{s}_{1} \ldots \mathbf{s}_{N}}^{V}} \times \widehat{V}_{X ; \mathbf{s}_{1} \ldots \mathbf{s}_{N}}^{\prime V},
$$

with notation as in (5.7) for $H=H_{X}^{V}$.

By (5.8), the groups of deck transformations of these regular coverings are

$$
\operatorname{Deck}\left(\pi_{X ; \mathbf{s}_{1} \ldots \mathbf{s}_{N}}^{\prime V}\right)=\mathcal{R}_{X ; \mathbf{s}_{1} \ldots \mathbf{s}_{N}}^{\prime V} \quad \text { and } \quad \operatorname{Deck}\left(\pi_{X ; \mathbf{s}_{1} \ldots \mathbf{s}_{N}}^{V}\right)=\mathcal{R}_{X ; \mathbf{s}_{1} \ldots \mathbf{s}_{N}}^{V},
$$

respectively. If $V$ is connected,

$$
\operatorname{Deck}\left(\pi_{X ; \mathbf{s}_{1} \ldots \mathbf{s}_{N}}^{V}\right) \approx \frac{H_{1}(V ; \mathbb{Z})_{X}}{\operatorname{gcd}(\mathbf{s}) H_{1}(V ; \mathbb{Z})_{X}} \times \operatorname{gcd}(\mathbf{s}) H_{1}(V ; \mathbb{Z})_{X}
$$

In general, the second group in (6.3) is different from $\mathcal{R}_{X}^{V}$ (contrary to an explicit statement in [7, Section 5] and the spirit of the description). In the $\ell_{1}, \ldots, \ell_{N}=0$ case, $\widehat{V}_{X ; \mathbf{s}_{1} \ldots \mathbf{s}_{N}}$ is a discrete set of points identified with $\mathcal{R}_{X}^{V}$. In most other cases, the coverings (6.1) are non-trivial (i.e. the first one is not $V_{\mathbf{s}_{1} \ldots \mathbf{s}_{N}} \times \mathcal{R}_{X ; \mathbf{s}_{1} \ldots \mathbf{s}_{N}}^{\prime V}$.

Suppose in addition that $V^{\prime} \subset V$ is the union of $V_{1}, \ldots, V_{N^{\prime}}$ for some $N^{\prime} \leq N$. By Corollary 3.3 , $H_{X}^{V^{\prime}} \subset H_{1}\left(V^{\prime} ; \mathbb{Z}\right)$ is the image of $H_{X}^{V}$ under the projection (5.10). Thus, the continuous map (5.11) with $H=H_{X}^{V}$ and $H^{\prime}=H_{X}^{V^{\prime}}$ induces a commutative diagram

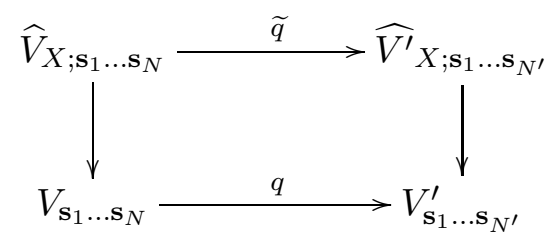

of fiber bundles. It corresponds to the right square in the diagram of Figure 1 . 
Example 6.1. Suppose $\widehat{\mathbb{P}}_{9}^{2}$ is a rational elliptic surface as in Example 3.5, $F \subset \widehat{\mathbb{P}}_{9}^{2}$ is a smooth fiber, $\ell \in \mathbb{Z}^{+}$, and $\mathbf{s} \in \mathbb{Z}^{\ell}$. In this case, $N=1, H_{X}^{V}=\{0\}$, and the first covering in (6.1) is isomorphic to the restriction of (5.12) to any of the connected components of $\mathbb{C} \times \mathbb{T}_{\mathbf{s}}^{2(n-1)}$. Its group of deck transformations is $\mathcal{R}_{\widehat{\mathbb{P}}_{9}^{2} ; \mathrm{s}}^{\prime F} \approx \mathbb{Z}^{2}$ and can be identified with

$$
\operatorname{gcd}(\mathbf{s}) \mathcal{R}_{\widehat{\mathbb{P}}_{9}^{2}}^{F} \subset \mathcal{R}_{\widehat{\mathbb{P}}_{9}^{2}}^{F} \approx \mathbb{Z}^{2}
$$

The second covering in (6.1) is (5.12) itself; its group of deck transformations is isomorphic to $\left(\mathbb{Z}_{\text {gcd }(\mathbf{s})}\right)^{2} \oplus \mathbb{Z}^{2}$.

Example 6.2. Let $F, X$, and $F_{0}, F_{\infty} \subset X$ be as in Example 3.6 with $F$ connected and $V=F_{0} \cup F_{\infty}$. In this case, $N=2$,

$$
H_{1}(V ; \mathbb{Z})=H_{1}(F ; \mathbb{Z}) \oplus H_{1}(F ; \mathbb{Z}),
$$

and $H_{X}^{V} \subset H_{1}(V ; \mathbb{Z})$ is the diagonal. With the identifications of Example 3.6, the composition of the homomorphism (5.2) with the projection to $\mathcal{R}_{X}^{V}$ can be written as

$$
H_{1}(F ; \mathbb{Z})^{\ell_{1}} \oplus H_{1}(F ; \mathbb{Z})^{\ell_{2}} \longrightarrow H_{1}(F ; \mathbb{Z}), \quad\left(\left(\gamma_{0 ; i}\right)_{i \leq \ell_{1}},\left(\gamma_{\infty ; i}\right)_{i \leq \ell_{2}}\right) \longrightarrow \sum_{i=1}^{\ell_{1}} s_{1 ; i} \gamma_{0 ; i}-\sum_{i=1}^{\ell_{2}} s_{2 ; i} \gamma_{\infty ; i}
$$

The first covering in (6.1) is thus the first covering in (5.6) with $V$ replaced by $F, H=\{0\}$, and $\mathbf{s}$ being the merged tuple of $\mathbf{s}_{1}$ and $-\mathbf{s}_{2}$. If $F=\mathbb{T}^{2}$ and $\left(\ell_{1}, \ell_{2}\right) \neq \mathbf{0}$, the second covering in (6.1) is described by (5.12) with $\mathbf{s}$ replaced by the merged tuple of $\mathbf{s}_{1}$ and $-\mathbf{s}_{2}$. With $V^{\prime}=F_{0}$ in (6.4), $\widehat{V}_{X ; \mathbf{s}_{1}}=V^{\ell_{1}}$ and $\widetilde{q}=q$.

If $\Sigma$ is a compact oriented $m$-dimensional manifold, $A \in H_{m}(X ; \mathbb{Z}), k \in \mathbb{Z}^{\geq 0}$, and $p>m$, let $\mathfrak{X}_{\Sigma, k}(X, A)$ be the space of tuples $\left(z_{1}, \ldots, z_{k}, f\right)$ such that $f \in L_{1}^{p}(\Sigma ; X), f_{*}[\Sigma]=A$, and $z_{1}, \ldots, z_{k} \in \Sigma$ are distinct points. If in addition $m=\mathfrak{c}, V_{1}, \ldots, V_{N}$ and $\mathbf{s}_{1}, \ldots, \mathbf{s}_{N}$ are as before, and (1.1) holds for each $(V, \mathbf{s})=\left(V_{r}, \mathbf{s}_{r}\right)$, let

$$
\mathfrak{X}_{\Sigma, k ; \mathbf{s}_{1} \ldots \mathbf{s}_{N}}^{V_{1}, \ldots, V_{N}}(X, A) \subset \mathfrak{X}_{\Sigma, k+\ell_{1}+\ldots+\ell_{N}}(X, A)
$$

be the subspace of tuples $\left(z_{1}, \ldots, z_{k+\ell_{1}+\ldots+\ell_{N}}, f\right)$ such that

$$
\begin{array}{ll}
f^{-1}\left(V_{r}\right)=\left\{z_{k+\ell_{1}+\ldots+\ell_{r-1}+1}, \ldots, z_{k+\ell_{1}+\ldots+\ell_{r}}\right\} & \forall r=1, \ldots, N, \\
\operatorname{ord}_{z_{k+\ell}+\ldots+\ell_{r-1}+i}^{V_{r}} f=s_{r ; i} & \forall i=1,2, \ldots, \ell_{r}, r=1, \ldots, N .
\end{array}
$$

We denote by

$$
\operatorname{ev}_{X}^{V}=\operatorname{ev}_{k+1} \times \ldots \times \operatorname{ev}_{k+\ell_{1}+\ldots+\ell_{N}}: \mathfrak{X}_{\Sigma, k ; \mathbf{s}_{1} \ldots \mathbf{s}_{N}}^{V_{1}, \ldots, V_{N}}(X, A) \longrightarrow V_{\mathbf{s}_{1} \ldots \mathbf{s}_{N}}
$$

the total relative evaluation morphism. The $\mathfrak{c}=2$ case of the next lemma is the implied claim of $[7$, Section 5].

Lemma 6.3. Suppose $X$ is a compact oriented manifold, $V \subset X$ is a compact oriented submanifold of codimension $\mathfrak{c}$ with connected components $V_{1}, \ldots, V_{N}, A \in H_{\mathfrak{c}}(X ; \mathbb{Z})$, and $\mathbf{s}_{r} \in \mathbb{Z}_{ \pm}^{\ell_{r}}$ for $r=1, \ldots, N$. If $\Sigma$ is a compact oriented $\mathfrak{c}$-dimensional manifold, $k \in \mathbb{Z}^{\geq 0}$, and $r=1, \ldots, N$, then the morphism (6.5) lifts over the first covering in (6.1) to a continuous map

$$
\widetilde{\mathrm{ev}}_{X}^{\prime V}: \mathfrak{X}_{\Sigma, k ; \mathbf{s}_{1} \ldots \mathbf{s}_{N}}^{V_{1}, \ldots, V_{N}}(X, A) \longrightarrow \widehat{V}_{X ; \mathbf{s}_{1} \ldots \mathbf{s}_{N}}^{\prime}
$$


Proof. Let

$$
\gamma:[0,1] \longrightarrow \mathfrak{X}_{\Sigma, k ; \mathbf{s}_{1} \ldots \mathbf{s}_{N}}^{V_{1}, \ldots, V_{N}}(X, A), \quad t \longrightarrow\left(z_{t ; 1}, \ldots, z_{t ; k+\ell_{1}+\ldots+\ell_{N}}, f_{t}\right)
$$

be a loop. For each $r=1, \ldots, N$ and $i=1, \ldots, \ell_{r}$,

$$
\gamma_{r ; i} \equiv \mathrm{ev}_{k+\ell_{1}+\ldots+\ell_{r-1}+i} \circ \gamma:[0,1] \longrightarrow V_{r}
$$

is also a loop. For each $i=1, \ldots, \ell_{1}+\ldots+\ell_{N}$, let $B_{z_{k+i}} \subset \Sigma$ be a small ball around $z_{k+i}$. As in the construction of $f \#\left(-f^{\prime}\right)$ in Section 2.1, it can be assumed that

$$
f_{t}:\left.\partial B_{z_{k+\ell_{1}+\ldots+\ell_{r-1}+i}} \longrightarrow S_{X} V\right|_{f_{t}\left(z_{k+\ell_{1}+\ldots+\ell_{r-1}+i}\right)} .
$$

Since $\gamma$ is a loop,

$$
\begin{aligned}
0=\left[f_{1} \#\left(-f_{0}\right)\right] & =\sum_{r=1}^{N} \sum_{i=1}^{\ell_{r}} \iota_{S_{X} V_{r} *}^{X-V}\left(\Delta_{X}^{V_{r}}\left(s_{r ; i} \gamma_{r ; i}\right)\right) \\
& =\iota_{S_{X} V *}^{X-V}\left(\Delta_{X}^{V}\left(\Phi_{V ; \mathbf{s}_{1} \ldots \mathbf{s}_{N}}\left(\operatorname{ev}_{X}^{V} \circ \gamma\right)\right) \in \mathcal{R}_{X}^{V} .\right.
\end{aligned}
$$

By Corollary 3.2, this implies that $\Phi_{V ; \mathbf{s}_{1} \ldots \mathbf{s}_{N}}\left(\operatorname{ev}_{X}^{V} \circ \gamma\right) \in H_{X}^{V}$. Thus, the image of the fundamental group of $\mathfrak{X}_{\Sigma, k ; \mathbf{s}_{1} \ldots \mathbf{s}_{N}}^{V_{1}, \ldots, V_{N}}(X, A)$ under (6.5) in $\pi_{1}\left(V_{\mathbf{s}_{1} \ldots \mathbf{s}_{N}}\right)$ lies in the image of the fundamental group of $\widehat{V}_{X ; \mathbf{s}_{1} \ldots \mathbf{s}_{N}}^{\prime}$ under $\pi_{X ; \mathbf{s}_{1} \ldots \mathbf{s}_{N}}^{\prime V}$. By [16, Lemma 79.1], this implies the claim.

Proof of Theorem 1.2. Since $\operatorname{gcd}(\mathbf{s})$ and $\left|\mathcal{R}_{X}^{V}\right|$ are relatively prime, $\widehat{V}_{H ; \mathbf{s}}=\widehat{V}_{H ; \mathbf{s}}^{\prime}$. The second inclusion in (1.12) is then an equality by Corollary 5.5] (2)] with $H=H_{X}^{V}$. Under the additional assumption (1.11), both inclusions in (1.12) are equalities by Corollary 5.5. (3).

Remark 6.4. Let $V$ be possibly disconnected with topological components $V_{1}, \ldots, V_{N}$. The conclusions of Theorem 1.2 then hold if (1.10) and the relatively prime condition are replaced by

$$
\left\{\sum_{\ell_{r} \neq 0}\left[\gamma_{r}\right]_{H_{X}^{V}}: \gamma_{r} \in \operatorname{Flux}\left(V_{r}\right)_{X} \forall r\right\}=\operatorname{Flux}(V)_{X} \quad \text { and } \quad \mathcal{R}_{X ; \mathbf{s}_{1} \ldots \mathbf{s}_{N}}^{\prime V}=\mathcal{R}_{X}^{V},
$$

respectively. The latter is the case if $\operatorname{gcd}\left(\mathbf{s}_{r}\right)$ and $\left|H_{1}\left(V_{r} ; \mathbb{Z}\right)\right|$ are relatively prime for every $r$. It is also the case if $F$ is connected, $X=\mathbb{P}^{1} \times F$ and $V=\{0, \infty\} \times F$ as in Example 6.2, and $\operatorname{gcd}\left(\mathbf{s}_{1}, \mathbf{s}_{2}\right)$ and $\left|H_{1}(F ; \mathbb{Z})\right|$ are relatively prime. The conclusion of Proposition 1.3 holds if $V_{r} \approx \mathbb{T}^{2 n-2}$ for every $r=1, \ldots, N$ and $(6.6)$ holds.

\subsection{Consistent choices of lifts}

We next show that the lifts in Lemma 6.3 can be chosen in a systematic way, consistent with their use in [8] for refining the symplectic sum formula for GW-invariants and with the diagram in Figure 1, The significance of Theorem 6.5 for the former is demonstrated by [4, Proposition 4.2]. We continue with the notation of Section 6.1.

Theorem 6.5. Suppose $X$ is a compact oriented manifold, $V \subset X$ is a compact oriented submanifold of codimension $\mathfrak{c}$ with connected components $V_{1}, \ldots, V_{N}, A \in H_{\mathfrak{c}}(X ; \mathbb{Z})$, and $\mathbf{s}_{r} \in \mathbb{Z}_{ \pm}^{\ell_{r}}$ for $r=1, \ldots, N$. Let $\left\{\gamma_{j}\right\} \subset H_{1}(V ; \mathbb{Z})$ be a collection of representatives for the elements of $\mathcal{R}_{X}^{V} / \mathcal{R}_{X ; \mathbf{s}_{1} \ldots \mathbf{s}_{N}}^{\prime V}$. If $\Sigma$ is a compact oriented $\mathfrak{c}$-dimensional manifold and $k \in \mathbb{Z}^{\geq 0}$, there exists a lift

$$
\widetilde{\mathrm{ev}}_{X}^{V}: \mathfrak{X}_{\Sigma, k ; \mathbf{s}_{1} \ldots \mathbf{s}_{N}}^{V_{1}, \ldots, V_{N}}(X, A) \longrightarrow \widehat{V}_{X ; \mathbf{s}_{1} \ldots \mathbf{s}_{N}}
$$


of the morphism $\mathrm{ev}_{X}^{V}$ in (6.5) over the covering $\pi_{X ; \mathbf{s}_{1} \ldots \mathbf{s}_{N}}^{V}$ in (6.1) with the following property. For any $\mathbf{f}, \mathbf{f}^{\prime} \in \mathfrak{X}_{\Sigma, k ; \mathbf{s}_{1} \ldots \mathbf{s}_{N}}^{V_{1}, \ldots, V_{N}}(X, A)$ with

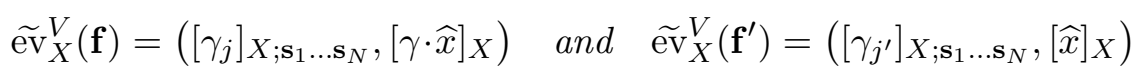

for some $\widehat{x} \in \widehat{V}_{\mathbf{s}_{1} \ldots \mathbf{s}_{N}}, \gamma \in H_{1}\left(V_{\mathbf{s}_{1} \ldots \mathbf{s}_{N}} ; \mathbb{Z}\right)$, and $j, j^{\prime}$ indexing the coset representatives, the map components of $\mathbf{f}$ and $\mathbf{f}^{\prime}$ satisfy

$$
\left[f \#\left(-f^{\prime}\right)\right]=\iota_{S_{X} V *}^{X-V}\left(\Delta_{X}^{V}\left(\Phi_{V ; \mathbf{s}_{1} \ldots \mathbf{s}_{N}}(\gamma)+\gamma_{j}-\gamma_{j^{\prime}}\right)\right) \in H_{\mathfrak{c}}(X-V ; \mathbb{Z}) .
$$

Furthermore, $\widetilde{\mathrm{ev}}_{X}^{V}\left(\mathbf{f}^{\prime}\right)$ is the unique point in $\pi_{X ; \mathbf{s}_{1} \ldots \mathbf{s}_{N}}^{V-1}\left(\operatorname{ev}_{X}^{V}\left(\mathbf{f}^{\prime}\right)\right)$ so that (6.9) holds for a given value of $\widetilde{\operatorname{ev}}_{X}^{V}(\mathbf{f})$.

Proof. We can assume that $\mathfrak{X}_{\Sigma, k ; \mathbf{s}_{1} \ldots \mathbf{s}_{N}}^{V_{1}, \ldots, V_{N}}(X, A) \neq \emptyset$; otherwise, there is nothing to prove. Choose a base point $\widehat{x}_{\mathbf{s}_{1} \ldots \mathbf{s}_{N}} \in \widehat{V}_{\mathbf{s}_{1} \ldots \mathbf{s}_{N}}$ over some $x_{\mathbf{s}_{1} \ldots \mathbf{s}_{N}} \in V_{\mathbf{s}_{1} \ldots \mathbf{s}_{N}}$, an overall base map

$$
\mathbf{f}_{0} \equiv\left(z_{0 ; 1}, \ldots, z_{0 ; k+\ell_{1}+\ldots+\ell_{N}}, f_{0}\right) \in \mathfrak{X}_{\Sigma, k ; \mathbf{s}_{1} \ldots \mathbf{s}_{N}}^{V_{1}, \ldots, V_{N}}(X, A) \quad \text { s.t. } \quad \operatorname{ev}_{X}^{V}\left(\mathbf{f}_{0}\right)=x_{\mathbf{s}_{1} \ldots \mathbf{s}_{N}},
$$

and a base point

$$
\mathbf{f}_{m} \equiv\left(z_{m ; 1}, \ldots, z_{m ; k+\ell_{1}+\ldots+\ell_{N}}, f_{m}\right) \in \mathfrak{X}_{\Sigma, k ; \mathbf{s}_{1} \ldots \mathbf{s}_{N}}^{V_{1}, \ldots, V_{N}}(X, A)
$$

for each topological component so that

$$
\operatorname{ev}_{X}^{V}\left(\mathbf{f}_{m}\right)=x_{\mathbf{s}_{1} \ldots \mathbf{s}_{N}}, \quad\left[f_{m} \#\left(-f_{0}\right)\right]=\iota_{S_{X} V *}^{X-V}\left(\Delta_{X}^{V}\left(\gamma_{j}\right)\right) \in H_{\mathfrak{c}}(X-V ; \mathbb{Z})
$$

for some $j=j(m)$. We define

$$
\widetilde{\mathrm{ev}}_{X}^{V}: \mathfrak{X}_{\Sigma, k ; \mathbf{s}_{1} \ldots \mathbf{s}_{N}}^{V_{1}, \ldots, V_{N}}(X, A) \longrightarrow \widehat{V}_{X ; \mathbf{s}_{1} \ldots \mathbf{s}_{N}} \equiv \frac{\mathcal{R}_{X}^{V}}{\mathcal{R}_{X ; \mathbf{s}_{1} \ldots \mathbf{s}_{N}}^{\prime V}} \times \widehat{V}_{X ; \mathbf{s}_{1} \ldots \mathbf{s}_{N}}^{\prime}
$$

to be the lift of $\operatorname{ev}_{X}^{V}$ over $\pi_{X ; \mathbf{s}_{1} \ldots \mathbf{s}_{N}}^{V}$ so that

$$
\widetilde{\mathrm{ev}}_{X}^{V}\left(\mathbf{f}_{m}\right)=\left(\left[\gamma_{j(m)}\right]_{X ; \mathbf{s}_{1} \ldots \mathbf{s}_{N}},\left[\widehat{x}_{\mathbf{s}_{1} \ldots \mathbf{s}_{N}}\right]_{X}\right) \quad \forall m ;
$$

see [16, Lemma 79.1].

It remains to verify that this lift has the claimed properties. Suppose $\mathbf{f}, \mathbf{f}^{\prime} \in \mathfrak{X}_{\Sigma, k ; \mathbf{s}_{1} \ldots \mathbf{s}_{N}}^{V_{1}, \ldots, V_{N}}(X, A)$ satisfy (6.8) and thus $\operatorname{ev}_{X}^{V}(\mathbf{f})=\operatorname{ev}_{X}^{V}\left(\mathbf{f}^{\prime}\right)$. Let $\mathbf{f}_{m}$ and $\mathbf{f}_{m^{\prime}}$ be the base points of the topological components of $\mathfrak{X}_{\Sigma, k ; \mathbf{s}_{1} \ldots \mathbf{s}_{N}}^{V_{1}, \ldots, V_{N}}(X, A)$ containing $\mathbf{f}$ and $\mathbf{f}^{\prime}$, respectively, and $\alpha$ and $\alpha^{\prime}$ be paths from $\mathbf{f}_{m}$ to $\mathbf{f}$ and from $\mathbf{f}_{m^{\prime}}$ to $\mathbf{f}^{\prime}$, respectively. By (6.8), $j(m)=j$ and $j\left(m^{\prime}\right)=j^{\prime}$. Along with (6.11), this gives

$$
\left[f_{m} \#\left(-f_{m^{\prime}}\right)\right]=\left[f_{m} \#\left(-f_{0}\right)\right]-\left[f_{m^{\prime}} \#\left(-f_{0}\right)\right]=\iota_{S_{X} V *}^{X-V}\left(\Delta_{X}^{V}\left(\gamma_{j}-\gamma_{j^{\prime}}\right)\right) \in H_{\mathfrak{c}}(X-V ; \mathbb{Z}) .
$$

Since $\operatorname{ev}_{X}^{V}\left(\mathbf{f}_{m}\right)=\operatorname{ev}_{X}^{V}\left(\mathbf{f}_{m^{\prime}}\right)$ and $\operatorname{ev}_{X}^{V}(\mathbf{f})=\operatorname{ev}_{X}^{V}\left(\mathbf{f}^{\prime}\right)$,

$$
\gamma^{\prime} \equiv\left(-\operatorname{ev}_{X}^{V} \circ \alpha^{\prime}\right) *\left(\operatorname{ev}_{X}^{V} \circ \alpha\right):[0,1] \longrightarrow V_{r}
$$

is a well-defined loop. Furthermore,

$$
\left[f \#\left(-f^{\prime}\right)\right]-\left[f_{m} \#\left(-f_{m^{\prime}}\right)\right]=\iota_{S_{X} V *}^{X-V}\left(\Delta_{X}^{V}\left(\Phi_{V ; \mathbf{s}_{1} \ldots \mathbf{s}_{N}}\left(\gamma^{\prime}\right)\right)\right) \in H_{\mathfrak{c}}(X-V ; \mathbb{Z}) .
$$


Combining this statement with (6.13), we find that

$$
\left[f \#\left(-f^{\prime}\right)\right]=\iota_{S_{X} V *}^{X-V}\left(\Delta_{X}^{V}\left(\Phi_{V ; \mathbf{s}_{1} \ldots \mathbf{s}_{N}}\left(\gamma^{\prime}\right)+\gamma_{j}-\gamma_{j^{\prime}}\right)\right) \in H_{\mathfrak{c}}(X-V ; \mathbb{Z}) .
$$

Let $\widehat{\alpha}, \widehat{\alpha}^{\prime}, \widehat{\gamma}^{\prime}:[0,1] \longrightarrow \widehat{V}_{\mathbf{s}_{1} \ldots \mathbf{s}_{N}}$ be the lifts of $\operatorname{ev}_{X}^{V} \circ \alpha, \operatorname{ev}_{X}^{V} \circ \alpha^{\prime}, \gamma^{\prime}$ such that

$$
\widehat{\alpha}(0), \widehat{\alpha}^{\prime}(0)=\widehat{x}_{\mathbf{s}_{1}, \ldots, \mathbf{s}_{N}}, \quad \widehat{\gamma}^{\prime}(0)=\widehat{\alpha}^{\prime}(1) .
$$

Thus,

$$
[\widehat{\alpha}(1)]_{X}=\widetilde{\operatorname{ev}}_{X}^{\prime V}(\mathbf{f})=[\gamma \cdot \widehat{x}]_{X}, \quad\left[\widehat{\alpha}^{\prime}(1)\right]_{X}=\widetilde{\operatorname{ev}}_{X}^{\prime V}\left(\mathbf{f}^{\prime}\right)=[\widehat{x}]_{X}
$$

where $\widetilde{\mathrm{ev}}_{X}^{\prime V}$ is the composition of (6.12) with the projection to the second component. Since $\widehat{\alpha}=\widehat{\alpha}^{\prime} * \widehat{\gamma}^{\prime}:[0,1] \longrightarrow \widehat{V}_{\mathbf{s}_{1} \ldots \mathbf{s}_{N}}$,

$$
[\gamma \cdot \widehat{x}]_{X}=[\widehat{\alpha}(1)]_{X}=\left[\gamma^{\prime} \cdot \widehat{\alpha}^{\prime}(1)\right]_{X}=\left[\gamma^{\prime} \cdot \widehat{x}\right]_{X}
$$

Since $\widehat{V}_{X ; \mathbf{s}_{1} \ldots \mathbf{s}_{N}}^{\prime}=\widehat{V}_{\mathbf{s}_{1} \ldots \mathbf{s}_{N}} / H_{X ; \mathbf{s}_{1} \ldots \mathbf{s}_{N}}^{V}$, it follows that

$$
\Phi_{V ; \mathbf{s}_{1} \ldots \mathbf{s}_{N}}(\gamma)-\Phi_{V ; \mathbf{s}_{1} \ldots \mathbf{s}_{N}}\left(\gamma^{\prime}\right) \in H_{X}^{V} \subset H_{1}(V ; \mathbb{Z}) .
$$

The claim (6.9) now follows from (6.14) and Corollary 3.2 with $U=\emptyset$. The latter also implies the uniqueness claim.

We will call a lift (6.7) satisfying the properties of Theorem 6.5 $\left\{\gamma_{j}\right\}$-compatible. The next proposition describes all such lifts.

Proposition 6.6. Suppose $X, V, \mathfrak{c}, A, \mathbf{s}_{r},\left\{\gamma_{j}\right\}, \Sigma$, and $k$ are as in the statement of Theorem 6.5.

(1) Let $x_{\mathbf{s}_{1} \ldots \mathbf{s}_{N}} \in V_{\mathbf{s}_{1} \ldots \mathbf{s}_{N}}$. If a lift (6.7) of (6.5) satisfies (6.9) for all $\mathbf{f}, \mathbf{f}^{\prime} \in \mathfrak{X}_{\Sigma, k ; \mathbf{s}_{1} \ldots \mathbf{s}_{N}}^{V_{1}, \ldots, V_{N}}(X, A)$ with $\operatorname{ev}_{X}^{V}(\mathbf{f})=x_{\mathbf{s}_{1} \ldots \mathbf{s}_{N}}$, then it is $\left\{\gamma_{j}\right\}$-compatible.

(2) Let $\eta \in H_{1}(X ; \mathbb{Z})$. If $\widetilde{\mathrm{ev}}_{X}^{V}$ is a lift of (6.5) compatible with $\left\{\gamma_{j}\right\}$, then so is the lift $\Theta_{\eta} \circ \widetilde{\mathrm{ev}}_{X}^{V}$.

(3) If the lifts $\widetilde{\mathrm{ev}}_{X}^{V}$ and $\widetilde{\mathrm{ev}}_{X}^{\prime V}$ as in (6.5) are compatible with $\left\{\gamma_{j}\right\}$, then $\widetilde{\mathrm{ev}}_{X}^{\prime V}=\Theta_{\eta} \circ \widetilde{\mathrm{ev}}_{X}^{V}$ for some $\eta \in H_{1}(X ; \mathbb{Z})$.

Proof. (1) This is immediate because the two sides of (6.9) take discrete values, but depend continuously on $\left(\mathbf{f}, \mathbf{f}^{\prime}\right)$.

(2)] If $\mathbf{f}, \mathbf{f}^{\prime} \in \mathfrak{X}_{\Sigma, k ; \mathbf{s}_{1} \ldots \mathbf{s}_{N}}^{V_{1}, \ldots, V_{N}}(X, A)$ satisfy (6.8), then

$$
\Theta_{\eta}\left(\widetilde{\mathrm{ev}}_{X}^{V}(\mathbf{f})\right)=\left(\left[\gamma_{j}(\eta)\right]_{X ; \mathbf{s}_{1} \ldots \mathbf{s}_{N}},\left[\eta_{j} \gamma \cdot \widehat{x}\right]_{X}\right), \quad \Theta_{\eta}\left(\widetilde{\operatorname{ev}}_{X}^{V}\left(\mathbf{f}^{\prime}\right)\right)=\left(\left[\gamma_{j^{\prime}}(\eta)\right]_{X ; \mathbf{s}_{1} \ldots \mathbf{s}_{N}},\left[\eta_{j^{\prime}} \cdot \widehat{x}\right]_{X}\right)
$$

with notation as in (5.9). By (5.9) and (6.9),

$$
\begin{aligned}
\iota_{S_{X} V *}^{X-V}\left(\Delta_{X}^{V}\left(\Phi_{V ; \mathbf{s}_{1} \ldots \mathbf{s}_{N}}\left(\eta_{j}+\gamma-\eta_{j^{\prime}}\right)+\gamma_{j}(\eta)-\gamma_{j^{\prime}}(\eta)\right)\right) & =\iota_{S_{X} V *}^{X-V}\left(\Delta_{X}^{V}\left(\Phi_{V ; \mathbf{s}_{1} \ldots \mathbf{s}_{N}}(\gamma)+\gamma_{j}-\gamma_{j^{\prime}}\right)\right) \\
& =\left[f \#\left(-f^{\prime}\right)\right] \in H_{\mathfrak{c}}(X-V ; \mathbb{Z}) .
\end{aligned}
$$


This establishes the second claim.

(3) Let $x_{\mathbf{s}_{1} \ldots \mathbf{s}_{N}}$ and $\mathbf{f}_{0}$ be as in (6.10). Suppose

$$
\widetilde{\mathrm{ev}}_{X}^{V}\left(\mathbf{f}_{0}\right)=\left(\left[\gamma_{j}\right]_{X ; \mathbf{s}_{1} \ldots \mathbf{s}_{N}},[\widehat{x}]_{X}\right), \quad \widetilde{\mathrm{ev}}_{X}^{\prime V}\left(\mathbf{f}_{0}\right)=\left(\left[\gamma_{j}^{\prime}\right]_{X ; \mathbf{s}_{1} \ldots \mathbf{s}_{N}},\left[\widehat{x}^{\prime}\right]_{X}\right)
$$

where $\gamma_{j}, \gamma_{j}^{\prime}$ are among the chosen coset representatives. Since $\tilde{\mathrm{ev}}_{X}^{V}$ and $\tilde{\mathrm{ev}}_{X}^{\prime V}$ are lifts of (6.5),

$$
\left[\widehat{x}^{\prime}\right]_{X}=\left[\eta_{j} \cdot \widehat{x}\right]_{X}
$$

for some $\eta_{j} \in H_{1}\left(V_{\mathbf{s}_{1} \ldots \mathbf{s}_{N}} ; \mathbb{Z}\right)$. Let

$$
\eta=\gamma_{j}^{\prime}-\gamma_{j}+\Phi_{V ; \mathbf{s}_{1} \ldots \mathbf{s}_{N}}\left(\eta_{j}\right)
$$

In particular, $\gamma_{j}(\eta)=\gamma_{j}^{\prime}$. Suppose $\mathbf{f}^{\prime} \in \mathfrak{X}_{\Sigma, k ; \mathbf{s}_{1} \ldots \mathbf{s}_{N}}^{V_{1}, \ldots, V_{N}}(X, A)$,

$$
\widetilde{\mathrm{ev}}_{X}^{V}\left(\mathbf{f}^{\prime}\right)=\left(\left[\gamma_{j^{\prime}}\right]_{X ; \mathbf{s}_{1} \ldots \mathbf{s}_{N}},\left[\gamma^{-1} \cdot \widehat{x}\right]_{X}\right), \quad \widetilde{\mathrm{ev}}_{X}^{\prime}\left(\mathbf{f}^{\prime}\right)=\left(\left[\gamma_{j^{\prime}}^{\prime}\right]_{X ; \mathbf{s}_{1} \ldots \mathbf{s}_{N}},\left[\eta_{j^{\prime}} \gamma^{-1} \cdot \widehat{x}\right]_{X}\right)
$$

for some $\gamma, \eta_{j^{\prime}} \in H_{1}\left(V_{\mathbf{s}_{1} \ldots \mathbf{s}_{N}} ; \mathbb{Z}\right)$. Since $\widetilde{\mathrm{ev}}_{X}^{V}$ and $\widetilde{\mathrm{ev}}_{X}^{\prime V}$ are lifts of (6.5) compatible with $\left\{\gamma_{j}\right\}$,

$$
\begin{aligned}
\iota_{S_{X} V *}^{X-V}\left(\Delta_{X}^{V}\left(\Phi_{V ; \mathbf{s}_{1} \ldots \mathbf{s}_{N}}(\gamma)+\gamma_{j}-\gamma_{j^{\prime}}\right)\right) & =\left[f_{0} \#\left(-f^{\prime}\right)\right] \\
& =\iota_{S_{X} V *}^{X-V}\left(\Delta_{X}^{V}\left(\Phi_{V ; \mathbf{s}_{1} \ldots \mathbf{s}_{N}}\left(\eta_{j}+\gamma-\eta_{j^{\prime}}\right)+\gamma_{j}^{\prime}-\gamma_{j^{\prime}}^{\prime}\right)\right) .
\end{aligned}
$$

It follows that

$$
\begin{gathered}
\gamma_{j^{\prime}}+\eta-\gamma_{j^{\prime}}^{\prime}-\Phi_{V ; \mathbf{s}_{1} \ldots \mathbf{s}_{N}}\left(\eta_{j^{\prime}}\right) \in H_{X}^{V}, \quad \gamma_{j^{\prime}}(\eta)=\gamma_{j^{\prime}}^{\prime}, \\
\Theta_{\eta}\left(\left[\gamma_{j^{\prime}}\right]_{X ; \mathbf{s}_{1} \ldots \mathbf{s}_{N}},\left[\gamma^{-1} \cdot \widehat{x}\right]_{X}\right)=\left(\left[\gamma_{j^{\prime}}^{\prime}\right]_{X ; \mathbf{s}_{1} \ldots \mathbf{s}_{N}},\left[\eta_{j^{\prime}} \gamma^{-1} \cdot \widehat{x}\right]_{X}\right) .
\end{gathered}
$$

Thus, $\widetilde{\mathrm{ev}}_{X}^{\prime V}=\Theta_{\eta} \circ \widetilde{\mathrm{ev}}_{X}^{V}$.

Remark 6.7. The above choices of $\left\{\gamma_{j}\right\}, \mathbf{f}_{0}$, and $\widehat{x}_{\mathbf{s}_{1} \ldots \mathbf{s}_{N}}$ roughly correspond to the two settheoretic descriptions of $\widehat{V}_{X ; \mathbf{s}_{1} \ldots \mathbf{s}_{N}}$ in [7] as equivalence classes of cycles in $X-V$ that are standard near $V$. These descriptions in [7, Section 5] do not specify a topology, especially when the contact points come together. A hands-on description of the topology of $\widehat{V}_{X ;(1)}$ is given at the end of [7, Section 5]; our definition of $\widehat{V}_{X ; \mathbf{s}_{1} \ldots \mathbf{s}_{N}}$ via the homomorphisms $\Phi_{V ; \mathbf{s}_{1} \ldots \mathbf{s}_{N}}$ is based on this description. Lemma 6.3 makes it apparent that the lifts of the evaluation maps can be extended over the compactified moduli spaces of relative stable maps once these spaces are shown to be compatible with gluing; the latter is necessary for any virtual construction of the moduli cycle and ensures that the boundary strata of the configuration spaces are of real codimension at least 2 (and so the loop $\gamma$ as in the proof of Lemma 6.3 can be assumed to lie completely in the main stratum).

Example 6.8. If $X=\widehat{\mathbb{P}}_{9}^{2}$ is a rational elliptic surface, $V=F$ is a smooth fiber as in Examples 3.5 and 6.1 , and $\ell \in \mathbb{Z}^{+}$, then $H_{X}^{V}=0$. We can identify $H_{1}(V ; \mathbb{Z})$ with $\mathbb{Z} \oplus \mathfrak{i} \mathbb{Z}$ and take

$$
x_{\mathbf{s}}=\left[\mathbf{0}_{2 \ell}\right] \in V^{\ell}=\mathbb{T}^{2 \ell}, \quad \widehat{x}_{\mathbf{s}}=\mathbf{0}_{2 \ell} \in \mathbb{C}^{\ell} .
$$

Let $\left\{\gamma_{j}\right\} \subset \mathbb{Z} \oplus \mathbf{i} \mathbb{Z}$ be a collection of representatives for the elements of $\mathbb{Z}_{\mathrm{gcd}(\mathbf{s})} \oplus \mathbf{i} \mathbb{Z}_{\mathrm{gcd}(\mathbf{s})}$. As indicated in [4, Example 3.1], it is convenient to identify the corresponding base points for the components of $\widehat{V}_{X ; \mathbf{s}}$ as

$$
\left(\left[\gamma_{j}\right]_{X ; \mathbf{s}},\left[\widehat{x}_{\mathbf{s}}\right]_{X}\right)=\left(\ell^{-1} \gamma_{j},\left[\ell^{-1} s_{i}^{-1} \gamma_{j}\right]_{i \leq \ell}\right) \in \widehat{V}_{X ; \mathbf{s}}=\mathbb{C} \times \mathbb{T}_{\mathbf{s}}^{2(\ell-1)} .
$$


Example 6.9. In the setting of Examples [3.6 and 6.2, only the tuples $\mathbf{s}_{1} \in \mathbb{Z}_{ \pm}^{\ell_{1}}$ and $\mathbf{s}_{2} \in \mathbb{Z}_{ \pm}^{\ell_{2}}$ with

$$
\sum_{i=1}^{\ell_{1}} s_{1 ; i}=\sum_{i=1}^{\ell_{2}} s_{2 ; i}
$$

are relevant in the context of Theorem 6.5. We first choose a base point $x_{1} \in F$. For all $\mathbf{s}_{1}$ and $\mathbf{s}_{2}$ as above, we then choose a base point $\widehat{x}_{\mathbf{s}_{1} \mathbf{s}_{2}}$ in $\widehat{F}_{\mathbf{S}}$ over $x_{1}^{\ell_{1}+\ell_{2}}$ in $F^{\ell_{1}+\ell_{2}}$, where $\mathbf{s}$ is the merged tuple of $\mathbf{s}_{1}$ and $\mathbf{s}_{2}$ as before, and a collection $\left\{\gamma_{j}\right\} \subset H_{1}(F ; \mathbb{Z})$ of representatives for the elements of

$$
\frac{H_{1}(V ; \mathbb{Z})_{X}}{\mathcal{R}_{X ; \mathbf{s}_{1} \mathbf{s}_{2}}^{\prime V}}=\frac{H_{1}(F ; \mathbb{Z})}{\operatorname{gcd}\left(\mathbf{s}_{1}, \mathbf{s}_{2}\right) H_{1}(F ; \mathbb{Z})}
$$

If $F=\mathbb{T}^{2}$ and $\left(\ell_{1}, \ell_{2}\right) \neq \mathbf{0}$, then we can identify $H_{1}(F ; \mathbb{Z})$ with $\mathbb{Z} \oplus \mathbf{Z}$. As indicated in [4, Example 3.3], it is then convenient to identify the corresponding base points for the components of $\widehat{V}_{X ; \text { s }}$ as

$$
\left(\left[\gamma_{j}\right]_{X ; \mathbf{s}_{1} \mathbf{s}_{2}},\left[\widehat{x}_{\mathbf{s}_{1} \mathbf{s}_{2}}\right]_{X}\right)=\left(\ell^{-1} \gamma_{j},\left(\left[\ell^{-1} s_{1 ; i}^{-1} \gamma_{j}\right]_{i \leq \ell_{1}},\left[-\ell^{-1} s_{2 ; i}^{-1} \gamma_{j}\right]_{i \leq \ell_{2}}\right)\right) \in \mathbb{C} \times \mathbb{T}_{\left(\mathbf{s}_{1},-\mathbf{s}_{2}\right)}^{2\left(\ell_{1}+\ell_{2}-1\right)}
$$

\subsection{Proof of Theorem 1.1}

Let $n_{V}=\operatorname{dim}_{\mathbb{R}} V$ and $r_{0}=\operatorname{rk}_{\mathbb{Z}} H_{1}(V ; \mathbb{Z})_{X}$. In light of (1.7) and (1.8), it is sufficient to show that

$$
\widehat{V}_{X ; \mathbf{s}}^{\prime} \approx \mathbb{R}^{r_{0}} \times Y
$$

for some manifold $Y$ of dimension $n_{V} \ell-r_{0}$.

With $B=\left(S^{1}\right)^{m}$ and $H_{X}^{V}$ as in (3.2), let

$$
H_{X}^{B}=q_{*}\left(H_{X}^{V}\right) \subset H_{1}(B ; \mathbb{Z}), \quad \widetilde{H}_{X}^{B}=q_{*}^{-1}\left(H_{X}^{B}\right) \subset H_{1}(V ; \mathbb{Z}) .
$$

Denote by $\mathcal{K}_{X ; \mathbf{s}}^{B} \subset \pi_{1}\left(B^{\ell}\right)$ and $\mathcal{K}_{X ; \mathbf{s}}^{V}, \widetilde{\mathcal{K}}_{X ; \mathbf{s}}^{B} \subset \pi_{1}\left(V^{\ell}\right)$ the preimages of $H_{X}^{B}$ and $H_{X}^{V}, \widetilde{H}_{X}^{B}$ under the homomorphisms

$$
\pi_{1}\left(B^{\ell}\right) \stackrel{\operatorname{Hur}}{\longrightarrow} H_{1}\left(B^{\ell} ; \mathbb{Z}\right) \stackrel{\Phi_{B ; \mathbf{s}}}{\longrightarrow} H_{1}(B ; \mathbb{Z}) \quad \text { and } \quad \pi_{1}\left(V^{\ell}\right) \stackrel{\operatorname{Hur}}{\longrightarrow} H_{1}\left(V^{\ell} ; \mathbb{Z}\right) \stackrel{\Phi_{V ; \mathbf{s}}}{\longrightarrow} H_{1}(V ; \mathbb{Z}),
$$

with $\Phi_{B ; \mathbf{s}}$ and $\Phi_{V ; \mathbf{s}}$ as in (5.2). From the commutativity of the diagram

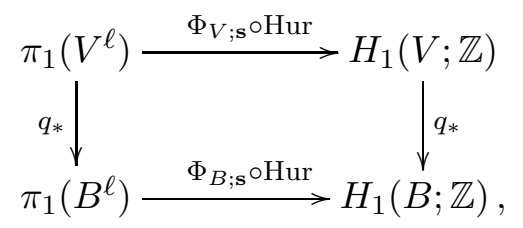

we find that $\widetilde{\mathcal{K}}_{X ; \mathbf{s}}^{B}=q_{*}^{-1}\left(\mathcal{K}_{X ; \mathbf{s}}^{B}\right)$.

Let

$$
\pi_{X ; \mathbf{s}}^{\prime B}: \widehat{B}_{X ; \mathbf{s}}^{\prime} \equiv \widehat{B}_{H_{X}^{B} ; \mathbf{s}}^{\prime} \longrightarrow B^{\ell} \quad \text { and } \quad \tilde{q}: \widetilde{V}_{X ; \mathbf{s}}^{B} \equiv \pi_{X ; \mathbf{s}}^{\prime B *} V^{\ell} \longrightarrow \widehat{B}_{X ; \mathbf{s}}^{\prime}
$$

be the covering corresponding to the normal subgroup $\mathcal{K}_{X ; \mathbf{s}}^{B}$ of $\pi_{1}\left(B^{\ell}\right)$ and the pullback of the fibration $q^{\ell}: V^{\ell} \longrightarrow B^{\ell}$, respectively. Since $\tilde{q}_{*}$ is surjective on $\pi_{1}$, the natural projection

$$
\tilde{\pi}_{X ; \mathbf{s}}^{B}: \widetilde{V}_{X ; \mathbf{s}}^{B} \longrightarrow V^{\ell}
$$


is the covering corresponding to the normal subgroup $q_{*}^{-1}\left(\mathcal{K}_{X ; \mathbf{s}}^{B}\right)=\widetilde{\mathcal{K}}_{X ; \mathbf{s}}^{B}$ of $\pi_{1}\left(V^{\ell}\right)$. Since $\mathcal{K}_{X ; \mathbf{s}}^{V} \subset \widetilde{\mathcal{K}}_{X ; \mathbf{s}}^{B}$, the total space of the covering

$$
\pi_{X ; \mathbf{s}}^{\prime V}: \widehat{V}_{X ; \mathbf{s}}^{\prime} \longrightarrow V_{\mathbf{s}}=V^{\ell}
$$

corresponding to $\mathcal{K}_{X ; \mathbf{s}}^{V}$ is also a covering of $\widetilde{V}_{X ; \mathbf{s}}^{B}$.

By the homotopy exact sequence [19, Theorem 7.2.10] for the fibration $q: V \longrightarrow B$ and the Hurewicz isomorphism [19, Theorem 7.5.5], the sequence

$$
H_{1}(F ; \mathbb{Z}) \longrightarrow H_{1}(V ; \mathbb{Z}) \stackrel{q_{*}}{\longrightarrow} H_{1}(B ; \mathbb{Z}) \longrightarrow 0
$$

is exact. Since $H_{1}(F ; \mathbb{Q})=\{0\}$,

$$
\operatorname{rk}_{\mathbb{Z}}\left(H_{1}(B ; \mathbb{Z}) / H_{X}^{B}\right)=\operatorname{rk}_{\mathbb{Z}} H_{1}(V ; \mathbb{Z})_{X}=r_{0} .
$$

Similarly to Example 5.1, this implies that

$$
\widehat{B}_{X ; \mathbf{s}}^{\prime} \approx \mathbb{R}^{r_{0}} \times\left(S^{1}\right)^{m \ell-r_{0}} .
$$

Thus, $\widetilde{V}_{X ; \mathbf{s}}^{B} \approx \mathbb{R}^{r_{0}} \times Y^{\prime}$ for some manifold $Y^{\prime}$ of dimension $n_{V} \ell-r_{0}$ and so (6.15) holds for some covering $Y$ of $Y^{\prime}$.

Remark 6.10. Theorem 1.1 extends to disconnected divisors $V$ with topological components $V_{1}, \ldots, V_{N}$ by replacing the rank of $H_{1}(V ; \mathbb{Z})_{X}$ with the rank of the submodule in (5.14) with $H=H_{X}^{V}$

Simons Center for Geometry and Physics, SUNY Stony Brook, NY 11794

mtehrani@scgp.stonybrook.edu

Department of Mathematics, SUNY Stony Brook, Stony Brook, NY 11794

azinger@math.sunysb.edu

\section{References}

[1] M. Artin, Algebra, Prentice Hall, 1991.

[2] K. Behrend and B. Fantechi, The intrinsic normal cone, Invent. Math. 128 (1997), no. 1, $45-88$.

[3] W. Dwyer and D. Fried, Homology of free abelian covers I, Bull. London Math. Soc. 19 (1987), no. $4,350-352$.

[4] M. Farajzadeh Tehrani and A. Zinger, On the refined symplectic sum formula for GromovWitten invariants, pre-print.

[5] K. Fukaya and K. Ono, Arnold conjecture and Gromov-Witten invariant, Topology 38 (1999), no. 5, 933-1048. 
[6] R. Gompf, A new construction of symplectic manifolds, Ann. of Math. 142 (1995), no. 3, $527-595$.

[7] E. Ionel and T. Parker, Relative Gromov-Witten invariants, Ann. of Math. 157 (2003), no. 1, 45-96.

[8] E. Ionel and T. Parker, The symplectic sum formula for Gromov-Witten invariants, Ann. of Math. 159 (2004), no. 3, 935-1025.

[9] J. Li, Stable morphisms to singular schemes and relative stable morphisms, J. Diff. Geom. 57 (2001), no. 3, 509-578.

[10] J. Li, A degeneration formula for GW-invariants, J. Diff. Geom. 60 (2002), no. 1, 199-293.

[11] A.-M. Li and Y. Ruan, Symplectic surgery and Gromov-Witten invariants of Calabi-Yau 3folds, Invent. Math. 145 (2001), no. 1, 151-218.

[12] J. Li and G. Tian, Virtual moduli cycles and Gromov-Witten invariants of general symplectic manifolds, in Topics in Symplectic 4-Manifolds, 47-83, Internat. Press 1998.

[13] D. McDuff and D. Salamon, Symplectic Topology, 2nd Ed., Oxford University Press, 1998.

[14] J. Milnor, Infinite cyclic coverings, in Conference on the Topology of Manifolds, 115-133, Prindle, Weber \& Schmidt, 1968.

[15] J. Milnor, note, 2014.

[16] J. Munkres, Topology: a first course, 2nd Ed., Pearson, 2000.

[17] J. Munkres, Elements of Algebraic Topology, Addison-Wesley 1984.

[18] J. McCarthy and J. Wolfson, Symplectic normal connect sum, Topology 33 (1994), no. 4, 729-764.

[19] E. Spanier, Algebraic Topology, Springer 1994.

[20] F. Warner, Foundations of Differentiable Manifolds and Lie Groups, GTM 94, Springer 1971.

[21] A. Zinger, Pseudocycles and integral homology, Trans. Amer. Math. Soc. 360 (2008), no. 5, $2741-2765$. 